\title{
The Simple Harmonic Oscillator
}

\section{Contents}

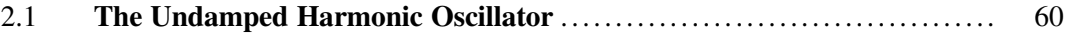

2.1.1 Initial Conditions and the Phasor Representation .................... 61

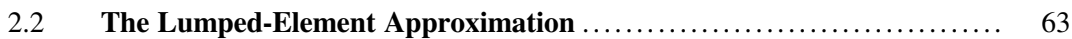

2.2.1 Series and Parallel Combinations of Several Springs .................... 64

2.2.2 A Characteristic Speed ...................................... 65

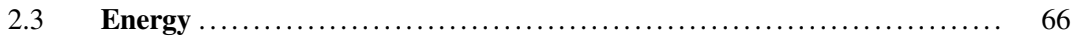

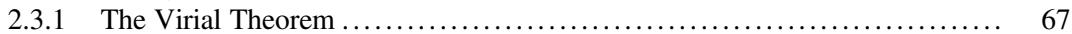

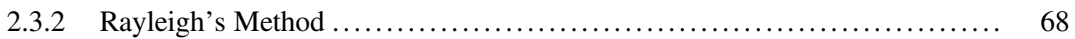

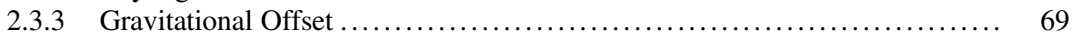

2.3.4 Adiabatic Invariance ............................................ 71

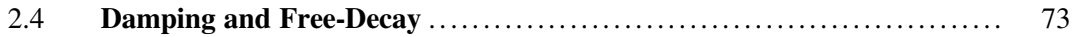

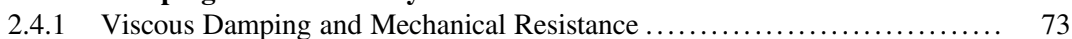

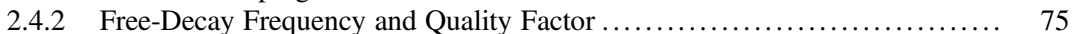

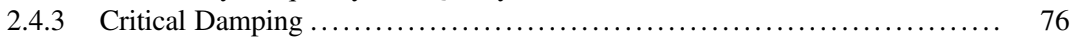

2.4.4 Thermal Equilibrium and Fluctuations ............................. 76

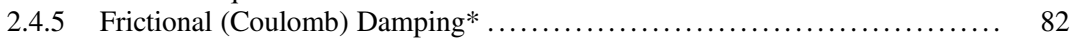

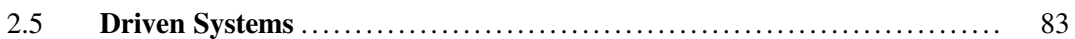

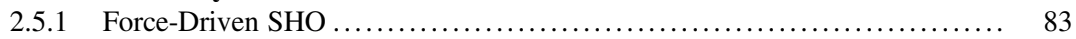

2.5.2 Power Dissipation, the Decibel, and Resonance Bandwidth ............. 87

2.5.3 Resonance Tracking and the Phase-Locked Loop* .................... 90

2.5.4 Transient Response .............................................. 92

2.5.5 The Electrodynamic Loudspeaker ................................. 95

2.5.6 Electrodynamic (Moving-Coil) Microphone ........................ 99

2.5.7 Displacement-Driven SHO and Transmissibility .................. 100

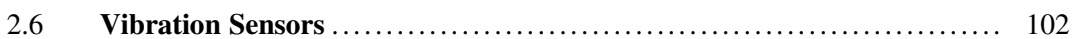

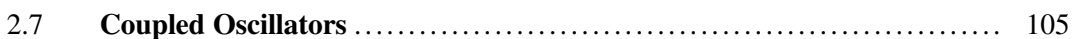

2.7.1 Two Identical Masses with Three Identical Springs .................... 105

2.7.2 Coupled Equations for Identical Masses and Springs ................ 106

2.7.3 Normal Modes and Normal Coordinates ........................... 107 


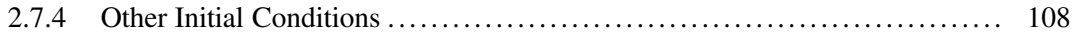

2.7.5 General Solutions for Two Masses and Three Springs .................... 109

2.7.6 Driven Oscillators, Level Repulsion, and Beating .................... 110

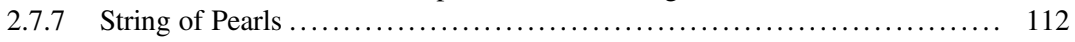

$2.8 \quad$ The Not-So-Simple (?) Harmonic Oscillator ..................... 115

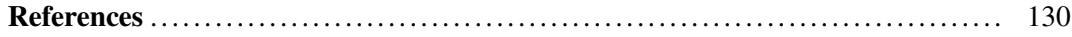

This chapter will introduce a system that is fundamental to our understanding of more physical phenomena than any other. Although the "simple" harmonic oscillator seems to be only the combination of the most mundane components, the formalism developed to explain the behavior of a mass, spring, and damper is used to describe systems that range in size from atoms to oceans.

The treatment of the harmonic oscillator in this chapter goes beyond the "traditional" treatments found in the elementary physics textbooks. For example, the introduction of damping will open a two-way street: a damping element (i.e., a mechanical resistance, $R_{m}$ ) will dissipate the oscillator's energy, thus reducing the amplitudes of successive oscillations, but it will also connect the oscillator to the surrounding environment (where the dissipated energy must go to "leave" the system). That surrounding environment will also return energy into the oscillator. The oscillation amplitude does not decay to zero but to a value that represents the oscillator's thermal equilibrium with its surroundings.

The excitation of such a harmonic oscillator by an externally applied force, displacement, or combination of the two will result in a response that is critically dependent upon the relationship between the frequency of excitation and the natural frequency of the oscillator, which is the oscillation of the undamped free oscillations. We will pay special attention to both the magnitude and phase of the driven response and will introduce the critical concepts of mechanical impedance, resonance, and quality factor.

Finally, the harmonic oscillator model will be extended to coupled oscillators that are represented by combinations of several masses and several springs. As the number of coupled masses and springs increases, several new features of such combinations will preview the behavior of continuous systems (like strings, bars, membranes, and plates). Ultimately, solid matter consists of a nearly infinite number of masses (atoms and molecules) that are coupled together by three times that number of stiffnesses arising from their mutual attraction or repulsion.

\subsection{The Undamped Harmonic Oscillator}

We begin this exploration by simply joining one point mass (meaning we neglect the spatial distribution of the mass which might require the specification of a moment of inertia), $m$, to one end of a spring with stiffness constant, $\mathrm{K}$, where the concept of a stiffness constant was addressed in Sect. 1.2.1 and the stiffness was related to potential gradients that produced forces or vice versa. The other end of the spring is assumed to be rigidly immobilized (i.e., fixed, as shown in Fig. 1.3). We further restrict the junction of the spring and the mass to move only along a single direction: the $x$ axis. This system is called a one-dimensional, single degree-of-freedom, undamped simple harmonic oscillator. (Damping will be added in Sect. 2.4.) Why does such a simple combination of two idealized components have such a complicated designation? It is an indication that there will be numerous variations on this theme.

At this stage, we will assume that the spring is massless, $m_{s}=0$, but we will see soon that a non-zero spring mass can be easily accommodated under some simplifying assumptions. Our analysis of the motion of this simple mass-spring system will begin by combining a dynamical equation with an equation of state. The dynamical equation is Newton's Second Law of Motion. It relates the 
acceleration of the mass to the net external force acting on the mass. The net force will be that which the spring exerts on the mass, determined by the displacement of the spring, $x$, from its equilibrium extension, $x_{o}$, according to Hooke's law in Eq. (1.25). You may be more familiar with thermodynamic equations of state that relate the pressure of a gas to its density or temperature, but Hooke's law is a simple example of an equation of state that relates some deformation of the system (a stimulus) to its response (the net force). The dynamical equation is generic (i.e., system independent), but the equation of state is particular to a specific system, the spring in this case. To simplify subsequent calculations, we will define the origin of our one-dimensional coordinate system to be located at $x_{o}=0$, the equilibrium length of our spring:

$$
F=m a=m \frac{d^{2} x}{d t^{2}}=-\mathrm{K} x \quad \text { or } \quad \frac{d^{2} x}{d t^{2}}+\frac{\mathrm{K}}{m} x=0
$$

There is an assumption that is built into Hooke's law that will be examined in the next section. We are assuming that the restoring force produced at the spring's moveable end depends only on the position of that end. That is true for a static extension or compression (e.g., weighing bananas on a spring scale in a market or the Gerber scale in Fig. 2.2). It might not be exact in a dynamical situation since we have assumed that the entire spring is instantaneously aware of the end's location. For that to be true, information about the end's position would have to propagate along the spring with an infinite speed, as discussed in Sect. 2.2.

Equation (2.1) is a second-order, homogeneous, linear differential equation with constant coefficients, and therefore there are two linearly independent solutions. As discussed in Sect. 1.5, we could solve that equation to provide the entire time history of the mass-spring system's response, $x(t)$, using a superposition of sine and cosine functions or complex exponentials, where we have set $\omega_{o}^{2}=\mathrm{K} / m$.

$$
x(t)=A \cos \left(\omega_{o} t\right)+B \sin \left(\omega_{o} t\right)
$$

This solution is periodic and repeats when $\omega_{o} T=2 \pi$ or integer multiples of $2 \pi$, so the frequency of oscillation, $f_{\mathrm{o}}=(1 / T)=\omega_{o} / 2 \pi$, is the reciprocal of the period, $T$.

$$
\omega_{o}=2 \pi f_{o}=\frac{2 \pi}{T}=\sqrt{\frac{\mathrm{K}}{m}}
$$

We call $\omega_{o}$ the angular frequency or the radian frequency. The radian frequency simplifies calculations by eliminating the need to explicitly include factors of $2 \pi$. It is important to remember that instrumentation and most software typically report frequencies in cycles per second $[\mathrm{Hz}]$, not radians per second.

\subsubsection{Initial Conditions and the Phasor Representation}

It is worthwhile to re-emphasize the fact that the "natural frequency" of oscillation, $\omega_{o}$, for this simple linear system (see Sect. 1.3) is amplitude independent. As shown in Sect. 1.7.1, simple dimensional arguments require that the frequency be proportional to $\sqrt{\mathrm{K} / m}$. The general solution for the motion of our simple undamped mass-spring oscillator in Eq. (2.2) contains two amplitude-dependent constants: $A$ and $B$. These constants are determined by the conditions that initiate the system's motion at the instant that defines $t=0$. We will first consider two ways that could set the system into motion: an initial displacement, $x_{1}$, or an initial impulse that propels the mass with an initial velocity, $v_{1} \equiv(\mathrm{d} x / \mathrm{d} t)_{\mathrm{o}}$, where the subscript on the derivative indicates that it is to be evaluated at $t=0$. 
If we hold the mass at position $x_{1}$ and then release it at $t=0$, the sine term vanishes, and the cosine term in Eq. (2.2) will equal one, so $A=x_{1}$. The instantaneous velocity of the mass is determined by differentiating Eq. (2.2):

$$
v(t)=\dot{x}(t)=\frac{d x(t)}{d t}=-\omega_{o} A \sin \left(\omega_{o} t\right)+\omega_{o} B \cos \left(\omega_{o} t\right)
$$

At $t=0$, the sine term again vanishes and $v(0)=\omega_{o} B$. Since the mass is at rest at $t=0, v(0)=0$, so $B=0$. For this initial displacement, the subsequent motion of the mass is given by $x(t)=x_{1} \cos \left(\omega_{o} t\right)$. Initially, the mass moves closer to its equilibrium position; one-quarter period later, it passes through its equilibrium position, $x_{o}=0$, reaching its maximum negative position, $-x_{1}$, a time, $T / 2$, after starting, and returns to its initial position, $x_{1}$, at $t=T$. In principle, this motion repeats forever with period, $T$.

If instead the mass is initially at rest at $x(0)=0$ and the motion is initiated by impulsively striking the mass to impart an initial velocity $v(0)=v_{1}$, then Eq. (2.4) sets $B=v_{1} / \omega_{o}$, while Eq. (2.2) sets $A=0$. The subsequent motion is given by $x(t)=\left(v_{1} / \omega_{o}\right) \sin \left(\omega_{o} t\right)$; we have given the mass an initial velocity in the $+x$ direction, and it moves off in that direction until reversing its direction one-quarter cycle later, after coming to rest for an instant at $x_{1}=\left|v_{1} / \omega_{o}\right|$.

Of course, those two exemplary initial conditions are not the only possibilities. In fact, we could impose both of the previous initial conditions simultaneously by displacing the mass by a distance, $x_{1}$, and then striking it at $t=0$ to produce a velocity $v_{1}$. The procedure to determine the amplitudes, $A$ and $B$, is the same and produces the same results.

$$
x(t)=x_{1} \cos \left(\omega_{o} t\right)+\frac{v_{1}}{\omega_{o}} \sin \left(\omega_{o} t\right)
$$

For an initial displacement in the $+x$ direction, combined with an initial velocity in the same direction, the amplitude of the oscillation will be larger than if either condition was imposed individually, as was done previously. A geometrical interpretation will be useful.

In Fig. 2.1, we imagine the position of the mass being represented by the projection on the $x$ axis of a vector that rotates counterclockwise at an angular velocity, $\omega_{o}$. The superposition of the two initial conditions produces a vector of length $|\vec{x}|=\sqrt{x_{1}^{2}+\left(v_{1} / \omega_{o}\right)^{2}}$. At $t=0$, the vector makes an angle $\phi=-\tan ^{-1}\left[\left(v_{1} / \omega_{o}\right) / x_{1}\right]$, which is below the $x$ axis in the fourth quadrant. As time increases, the vector rotates, and its projection along the $x$ axis increases until $t=\phi / \omega_{o}$ when the displacement reaches its maximum positive value.

Another way of expressing the solution provided in Eqs. (2.2) and (2.5) is to express $x(t)$ in terms of a single amplitude $C=\sqrt{x_{1}^{2}+\left(v_{1} / \omega_{o}\right)^{2}}$ and the same phase angle, $\phi$, as diagrammed in Fig. 2.1.

$$
x(t)=C \cos \left(\omega_{o} t+\phi\right)
$$

The value of $\phi$ allows a mixture of the appropriate proportion of the sine and cosine terms in Eq. (2.2) to meet the specific initial conditions. It also is ideally suited to the expression of $x(t)$ as the real part of a complex exponential.

$$
x(t)=\mathfrak{R e}\left[\widehat{\mathbf{C}} e^{j \omega_{o} t}\right]=\mathfrak{R e}\left[|\widehat{\mathbf{C}}| e^{j \phi} e^{j \omega_{o} t}\right]=\mathfrak{R e}\left[|\widehat{\mathbf{C}}| e^{j\left(\omega_{o} t+\phi\right)}\right]
$$

Now $\widehat{\mathbf{C}}$ is treated as a complex number (phasor) with magnitude $|\widehat{\mathbf{C}}|$ and phase $\phi$. Taking the real part of Eq. (2.7) regenerates Eq. (2.6) and allows the use of the complex exponential to simplify the calculation of integrals and derivatives as shown in Eq. (1.47). 


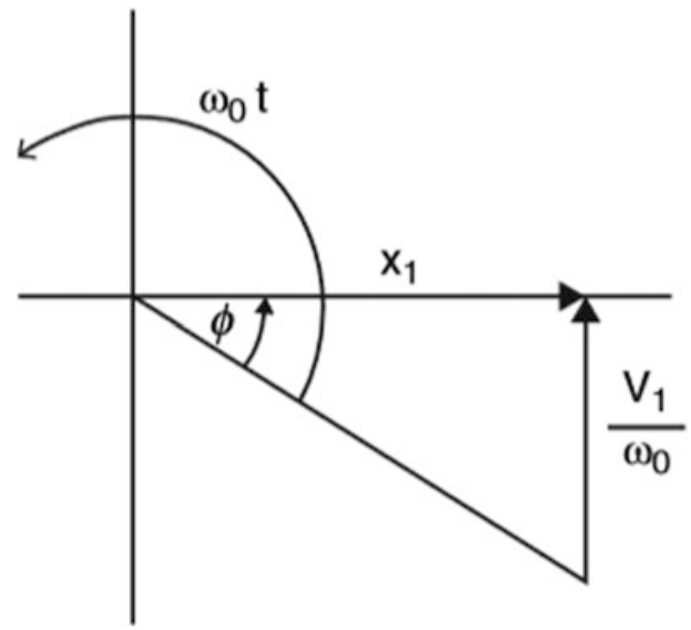

Fig. 2.1 Geometrical representation for the combination of an initial displacement, $x_{1}$, and initial velocity, $v_{1}$, for a single degree-of-freedom, undamped, simple harmonic oscillator. The vector of length $|\vec{x}|=\sqrt{x_{1}^{2}+\left(v_{1} / \omega_{o}\right)^{2}}$ starts at an angle $\phi=-\tan ^{-1}\left[\left(v_{1} / \omega_{o}\right) / x_{1}\right]$ and rotates in the counterclockwise direction at angular velocity, $\omega_{o}$. The projection of that vector on the horizontal axis represents the position of the mass as a function of time

For example, we can produce the velocity in complex notation, as we did for the trigonometric expression of Eq. (2.4), by simple multiplication of Eq. (2.7) by $j \omega_{o}$. Similarly, double differentiation of Eq. (2.7) produces the acceleration $a(t)$ by multiplication of Eq. (2.7) by $\left(j \omega_{o}\right)\left(j \omega_{o}\right)=-\omega_{o}^{2}$.

$$
\begin{aligned}
& v(t)=j \omega_{o} x(t)=\operatorname{Re}\left[j \omega_{o} \widehat{\mathbf{C}} e^{j \omega_{o} t}\right] \\
& a(t)=-\omega_{o}^{2} x(t)=\operatorname{Re}\left[-\omega_{o}^{2} \widehat{\mathbf{C}} e^{j \omega_{o} t}\right]
\end{aligned}
$$

These expressions indicate that there is a $+90^{\circ}$ phase shift (in the counterclockwise sense) between displacement and velocity and $180^{\circ}$ phase shift between displacement and acceleration.

The use of complex exponentials is so convenient and ubiquitous in the treatment of problems involving acoustics and vibration that it is customary to simply drop the explicit reference to "taking the real part of the complex expression" and treat the complex expressions as the "result" with the tacit understanding that the behavior of the physical system corresponds to only the projection of the complex vectors onto the real axis.

\subsection{The Lumped-Element Approximation}

The previous analysis of the undamped simple harmonic oscillator used a spring constant, $\mathrm{K}$, to relate the motion of the end of the spring to the force it imposed on the attached mass. The fact that the force depended only upon the position of the end of the spring implied a quasi-static approximation that is equivalent to saying that each location along the spring's entire length had instantaneous "knowledge" of the current location of the end of the spring. Said another way, information about the end position was instantaneously accessible at every location along the entire length of the spring, or the speed at which such information could propagate is infinite. In the quasi-static approximation, the displacement of each "coil" of the spring is linearly proportional to its distance from the fixed end. Figure 2.2 shows an adjustable scale (i.e., ruler) which exploits that proportionality. 


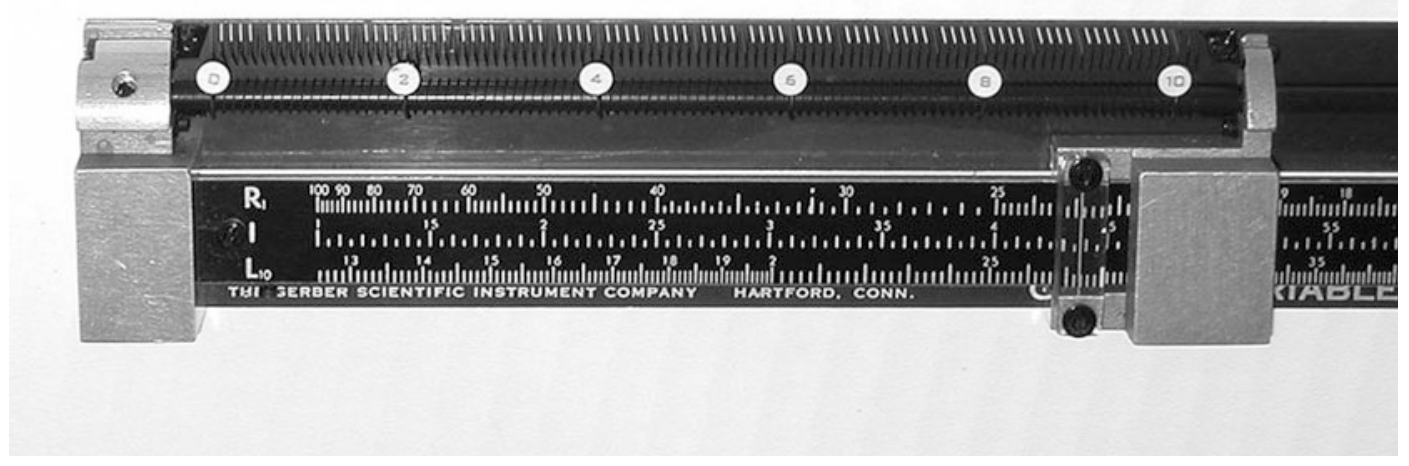

Fig. 2.2 The Gerber scale (H. Joseph Gerber (1924-1996) was a prolific inventor and able businessman who had been granted more than 650 patents over his lifetime) makes use of the quasi-static approximation to produce a ruler with equally spaced markings but adjustable overall length. At the top of the photograph is a triangular spring that has every tenth coil painted red, every fifth coil blue, and the other coils white. Even-numbered labels are mounted on a second spring to keep track of the red marks. Since these are linear springs, which obey Hooke's law, the coils will be equally spaced with respect to any stop position of the slider. Consequently, in the days before computerized plotting and data analysis, this scale could be used for linear interpolation or extrapolation of plotted data points without any computation. The logarithmic scale on the base plate allows it to be used on graphs with logarithmic coordinates

In reality, as the end of the spring is moved, there will be a wave launched from the moving end. Since we will not be addressing wave motion until the next chapter, we can make an estimate of the limitations of the quasi-static assumption using dimensional arguments (i.e., using similitude as discussed in Sect. 1.7). Before doing so, we need to consider the net stiffness of combinations of two or more springs.

\subsubsection{Series and Parallel Combinations of Several Springs}

If two springs are connected from a fixed mounting to the same load, as illustrated schematically on the left in Fig. 2.3, their individual stiffnesses will add. Springs combined in such an arrangement are said to be "springs in parallel." Since the ends of both springs will be displaced by the same amount, $x$, the magnitude of the net force, $F_{\text {parallel }}$, will be the sum of their forces.

$$
-F_{\text {parallel }}=-\left(F_{1}+F_{2}\right)=\mathrm{K}_{1} x+\mathrm{K}_{2} x=\left(\mathrm{K}_{1}+\mathrm{K}_{2}\right) x=\mathrm{K}_{\text {parallel }} x
$$

The parallel combination of the two springs of stiffness $\mathrm{K}_{1}$ and $\mathrm{K}_{2}$ will have an effective spring constant of $\mathrm{K}_{\text {parallel }}=\mathrm{K}_{1}+\mathrm{K}_{2}$. The extension to the parallel combination of several springs will result in an effective parallel spring constant that is just the sum of their individual stiffnesses.

If the same two springs are joined end to end, then we say the springs are combined "in series." Their effective series spring constant will be less than that of either spring acting alone. If the position of the free end of the combination is at $x$ and the junction is at $x_{a}$, then the forces at $x_{a}$ produced by either spring must be equal, since that intermediate position is neither accelerating nor decelerating.

$$
-F_{a}=\mathrm{K}_{1} x_{a}=\mathrm{K}_{2}\left(x-x_{a}\right) \quad \Rightarrow \quad x_{a}=\frac{\mathrm{K}_{2}}{\mathrm{~K}_{1}+\mathrm{K}_{2}} x
$$



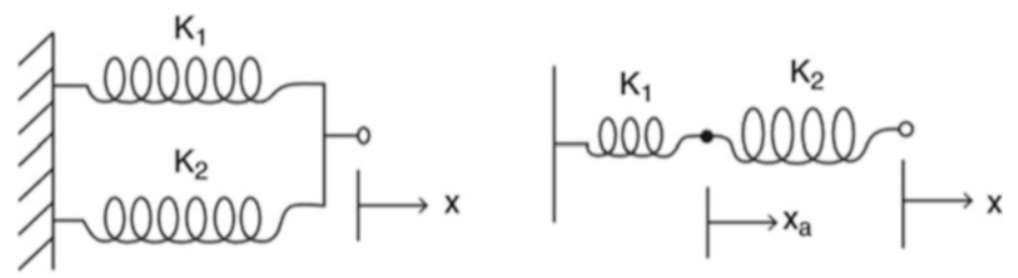

Fig. 2.3 (Left) Two springs combined in parallel will exhibit a net effective spring constant that is the sum of the individual spring constants. (Right) For the series combination, the forces at the junction between the two springs must be equal and opposite, as dictated by Newton's Third Law of Motion, and the net stiffness will be smaller than the stiffness of either spring

This result makes sense in the limit that $\mathrm{K}_{2} \gg \mathrm{K}_{1}$, making $\mathrm{K}_{2}$ effectively rigid, since only $\mathrm{K}_{1}$ would be extended if $x$ was increased so $x_{a} \cong x$. Similarly, in the opposite limit, if $\mathrm{K}_{1} \gg \mathrm{K}_{2}$, then only $\mathrm{K}_{2}$ would be extended, so $x_{a} \cong 0$. The other obvious check would be to let $\mathrm{K}_{1}=\mathrm{K}_{2}$. In that case, we expect each spring to provide half the displacement, i.e., $x_{a}=x / 2$, and the force would be half that of a single spring displaced from its equilibrium position by the same amount.

The force of the series combination can be obtained by substitution of $x_{a}$ back into the expression for the force.

$$
\begin{gathered}
-F_{a}=-F_{\text {series }}=\mathrm{K}_{1} x_{a}=\frac{\mathrm{K}_{1} \mathrm{~K}_{2}}{\mathrm{~K}_{1}+\mathrm{K}_{2}} x=\left(\frac{1}{\mathrm{~K}_{1}}+\frac{1}{\mathrm{~K}_{2}}\right)^{-1} x=\mathrm{K}_{\text {series }} x \\
\frac{1}{\mathrm{~K}_{\text {series }}}=\frac{1}{\mathrm{~K}_{1}}+\frac{1}{\mathrm{~K}_{2}}
\end{gathered}
$$

Again, the extension to a series concatenation of several springs is also simple. Readers with exposure to the basics of electrical circuit theory will recognize that these expressions for the stiffnesses of series and parallel combinations of springs are the exact reverse of those for the series and parallel combinations of electrical resistors or inductors, although the same as for series and parallel combinations of capacitors. This is because for electrical resistors in series, the flow (i.e., electrical current) is continuous and the potentials (i.e., voltages) are additive. For springs in series, the potential (i.e., force) is continuous, and the flow (i.e., displacement) is additive.

\subsubsection{A Characteristic Speed}

To determine an estimate for the limits of validity of the assumption that we can use the static stiffness of a spring for calculation of the dynamical behavior of a mass-spring oscillator, we can use a dimensional argument to calculate a characteristic speed for a spring of stiffness, $\mathrm{K}$, and length, $L$. Since such a characteristic speed should be a property of the spring and not any particular application, we need to identify spring properties that are not a function of the spring's length.

The spring's mass, $m_{s}$, can be divided by the spring's length, $L$, to produce a linear mass density, $\rho_{L}$, which is a characteristic of the spring and independent of length: $\rho_{L}=m_{s} / L$. For a uniform spring, $\rho_{L}$ will be a constant. Based on our calculation of the overall stiffness of a series combination of springs, the product of the stiffness, $\mathrm{K}$, of a spring with its length, $L$, is independent of the spring's length and only a property of the spring's construction (i.e., shape and materials). If two identical springs are attached in series, the overall stiffness of the combination is half that of either spring individually, and the overall length is doubled. For a uniform spring material, the product, $(L K)$, is a constant. 
We can now use similitude (see Sect. 1.7) to combine $\rho_{L}[\mathrm{~kg} / \mathrm{m}]$ and $(L K)\left[(\mathrm{N} / \mathrm{m}) \mathrm{m}=\mathrm{N}=\mathrm{kg}-\mathrm{m} / \mathrm{s}^{2}\right]$ to produce a speed, $c[\mathrm{~m} / \mathrm{s}]$, based on the units required to specify those two parameters that are characteristics of the spring and independent of its length.

$$
\rho_{L}^{a}(L \mathrm{~K})^{b}=\frac{M^{a}}{L^{a}} \frac{M^{b} L^{b}}{T^{2 b}}=\frac{L}{T}=c
$$

Since $c$ does not require any mass units, $a+b=0$. Since only (KL) involves $T, 2 b=1$, so $b=1 / 2$, and therefore $a=-1 / 2$. Equating the terms involving $L$ requires $-a+b=1$, which is also satisfied.

$$
c \propto \sqrt{\frac{L \mathrm{~K}}{\rho_{L}}}=L \sqrt{\frac{\mathrm{K}}{m_{s}}}
$$

To determine whether it was appropriate to use a static spring stiffness to solve a dynamical problem, we can now simply compare the period, $T$, of our simple harmonic oscillator given by Eq. (2.3) to the time, $t_{s}$, it takes for information to propagate back and forth along a spring of length, $L$, at speed $c: t_{s}=2 L / c$. If $T \gg t_{s}$, then there is plenty of time for the entire spring to be influenced by the changing position of its point of attachment to the mass [1].

The dimensional argument that produced Eq. (2.13) cannot provide any multiplicative numerical constant. For now, we will simply express the criterion, $T \gg t_{s}$, as $m \gg m_{s}$. Once we are able to calculate the speed of compressional wave propagation along the spring, we will see that our "lumpedelement" approximation, used to derive Eq. (2.3), is equivalent to saying that the spring length, $L$, is much less than the wavelength, $\lambda$, of a compressional wave of frequency, $f_{o}$. That criterion also leads to the requirement that $m \gg m_{s}$ to justify neglecting dynamical effects that would alter the effective stiffness of the spring from its static value.

To reiterate, under certain circumstances (usually at lower frequencies), it is possible to treat our mass as having no stiffness and our stiffness as having no mass, allowing us to neglect wavelike behavior associated with the spring's length. Under those assumptions, these components are specified at a point and are called "lumped elements" in the same way that inductors, capacitors, and resistors are considered lumped elements in electrical circuit theory.

\subsection{Energy}

Just as the definition of work was used to establish the relationship between a restoring force and its associated potential energy, Eq. (1.22) can be used to calculate the work done against the inertia of a particle with mass, $m$, based on Newton's Second Law of Motion: $\vec{F}=m(d \vec{v} / d t)$.

$$
W=-\int \vec{F} \cdot d \vec{x}=-m \int \frac{d v}{d t} d x=-m \int v d v=-\frac{1}{2} m v^{2}+\text { constant }
$$

A particle of mass, $m$, has an energy associated with its velocity, $v$, which is called its kinetic energy.

$$
K E=\frac{1}{2} m v^{2}
$$

The relationship between the work done by a conservative force and the change in potential energy created by such a vector force, $\vec{F}$, operating over a vector displacement, $\vec{x}$, was described in Sect. 1.2.1. If the force is produced by a linear spring that obeys Hooke's law, and the force and the displacement are co-linear, then the potential energy takes a particularly simple form. 


$$
P E=\frac{1}{2} \mathrm{~K} x^{2}
$$

In that expression, the displacement, $x$, is assumed to be the deviation from the spring's equilibrium position, $x_{o}$, so Eq. (2.16) also assumes a coordinate system with its origin making $x_{o}=0$.

If the origin of our time coordinate is defined so that we can eliminate the cosine term from the position of the mass, $x(t)$, expressed in Eq. (2.2), then we can write $x(t)=B \sin \left(\omega_{o} t\right)$ and $v(t)=\omega_{o} B \cos \left(\omega_{o} t\right)$. Substitution of these into the expressions for the potential and kinetic energy of a mass-spring system will provide an expression for the total instantaneous energy, $E_{T o t}(t)$.

$$
E_{T o t}(t)=K E(t)+P E(t)=\frac{m \omega_{o}^{2} B^{2}}{2} \cos ^{2}\left(\omega_{o} t\right)+\frac{\mathrm{K} B^{2}}{2} \sin ^{2}\left(\omega_{o} t\right)
$$

Since $\omega_{o}{ }^{2}=\mathrm{K} / m$, and $\cos ^{2} x+\sin ^{2} x=1$, the total energy is time-independent. The energy oscillates between being entirely kinetic, as the mass moves through $x(t)=0$, to entirely potential when $x(t)= \pm B$.

$$
E_{T o t}=\frac{\mathrm{K} B^{2}}{2}=\frac{m \omega_{o}^{2} B^{2}}{2}=(P E)_{\max }=(K E)_{\max }
$$

Furthermore, the time-averaged values of both the kinetic and potential energies are equal.

$$
\begin{aligned}
\langle K E\rangle_{t} & =\frac{m \omega_{o}^{2}}{2 T} \int_{0}^{T} B^{2} \cos ^{2}\left(\omega_{o} t\right) d t=\frac{m \omega_{o}^{2} B^{2}}{4} \\
\langle P E\rangle_{t} & =\frac{\mathrm{K}}{2 T} \int_{0}^{T} B^{2} \sin ^{2}\left(\omega_{o} t\right) d t=\frac{\mathrm{K} B^{2}}{4}
\end{aligned}
$$

Conservation of energy can be used to demonstrate the equivalence to the Newtonian formulation of Eq. (2.1).

$$
\frac{1}{2} m\left(\frac{d x}{d t}\right)^{2}+\frac{1}{2} \mathrm{~K} x^{2}=\text { constant }
$$

Differentiation of this expression with respect to time regenerates Eq. (2.1).

$$
\left[m \frac{d^{2} x}{d t^{2}}+\mathrm{K} x\right] \frac{d x}{d t}=0 \Rightarrow m \frac{d^{2} x}{d t^{2}}+\mathrm{K} x=0
$$

\subsubsection{The Virial Theorem}

The fact that the sum of the kinetic and potential energies is time-independent is a consequence of energy conservation, since no components have been introduced (yet!) in the system that can dissipate energy. The fact that the maximum and the time-averaged kinetic and potential energies are equal also seems plausible, but it is not universal. This equality is a consequence of the assumed Hooke's law behavior where the power law exponent, $b=1: F \propto x^{b}$.

For "central forces" (i.e., those forces that act along a line connecting the force with the object acted upon by that force) that obey a power law, the virial theorem guarantees that the time-averaged kinetic and potential energies are dependent upon that exponent [2]. 


$$
\langle K E\rangle_{t}=\frac{b+1}{2}\langle P E\rangle_{t}
$$

The equality of the time-averaged potential and kinetic energies is a consequence of our assumption of the linear behavior of our spring. It is useful to remember that this equality does not hold for a nonlinear spring.

A simple counterexample is planetary motion under the influence of gravity. In the simplest case of circular orbits, the centripetal force, $F_{c e n t}$, on a planet of mass, $m$, is provided by Newton's universal law of gravitation ${ }^{1}$ due to a larger central body of mass $M \gg m$. Their centers of mass are separated by a distance, $R$.

$$
F_{c e n t}=m \omega^{2} R=\frac{G M m}{R^{2}} \Rightarrow \omega^{2}=\frac{G M}{R^{3}}
$$

This is a result known as Kepler's third law of planetary motion: the square of the orbital period is proportional to the cube of the radius of the orbit. ${ }^{2}$ The planet's kinetic energy is determined by its tangential velocity, $v_{t}=\omega R$.

$$
K E=\frac{1}{2} m v_{t}^{2}=\frac{m}{2}(\omega R)^{2}=\frac{1}{2} \frac{G M m}{R}
$$

The gravitational force, $F_{\text {grav }}=G M m / R^{2}$, is the negative gradient of the gravitational potential energy.

$$
P E=-\frac{G M m}{R}
$$

For this simple example, both the $K E$ and $P E$ are time-independent, so the average $P E$ is twice the average $K E$ in accordance with Eq. (2.22) for $b=-2$.

\subsubsection{Rayleigh's Method}

"The object being to approach the truth as nearly as can be done without too great a sacrifice of simplicity."

(J. W. Strutt (Lord Rayleigh) [3])

We can take advantage of the equality of the time-averaged values of the kinetic and potential energy that is unique to the vibration of a system whose restoring force is linear in the displacement from equilibrium. The magnitude of the oscillatory velocity, $|v|$, for a simple harmonic oscillator is equal to the product of the magnitude of the displacement times the oscillatory frequency: $|v|=\omega_{o}|x|$. Setting $K E$ in Eq. (2.15) equal to $P E$ in Eq. (2.16) leads to the same expression for $\omega_{o}$ that was derived in Eq. (2.3).

This equality facilitates calculation of the correction to the natural frequency of the mass-spring oscillator when $m_{s} \neq 0$. The displacement of each coil of a spring that is extended from its equilibrium position, $x_{o}$, by an amount $x_{1}$ is proportional to its distance, $x$, from the fixed end at $x=0$, in the quasistatic approximation (as it was for the Gerber scale in Fig. 2.2). The magnitude of the displacement of

\footnotetext{
${ }^{1}$ The value of $G=6.6738 \times 10^{-11} \mathrm{~N} /(\mathrm{m} / \mathrm{kg})^{2}$ is the most poorly known physical constant, having a relative uncertainty of $\delta G / G= \pm 1.4 \times 10^{-4}$; P. J. Mohr, B. N. Taylor, and D. B. Newell, "The 2010 CODATA Recommended Values of the Fundamental Physical Constants," http://physics.nist.gov/constants

${ }^{2}$ Kepler's third law applies to elliptical orbits. The assumed circular orbit is just a special case for an ellipse without eccentricity. For an elliptical orbit, the square of the period is proportional to the cube of the semi-major axis.
} 
any point on the spring can be expressed as $\xi(x)=x_{1}(x / L)$. The magnitude of the velocity of the spring at that location is $v(x)=\omega_{o} \xi(x)$.

Again, defining the linear mass density, $\rho_{L}=m_{s} / L$, of the spring in terms of the spring's mass, $m_{s}$, and its length, $L$, as in Eq. (2.13), the kinetic energy of the spring $K E_{s}$ can be calculated by the mass density of the spring times the square of its maximum local velocity, $v^{2}(x)$, integrated over the entire length of the spring.

$$
\left(K E_{S}\right)_{\max }=\frac{1}{2} \int_{0}^{L} \rho_{L} v_{1}^{2}\left(\frac{x}{L}\right)^{2} d x=\frac{\omega_{o}^{2} x_{1}^{2}}{6}\left(L \rho_{L}\right)=\frac{1}{3}\left(\frac{1}{2} m_{s} \omega_{o}^{2} x_{1}^{2}\right)
$$

That result for the maximum kinetic energy of the spring can be added to the maximum kinetic energy of the mass attached to the mobile end of the spring. By Eq. (2.18), the total kinetic energy can be equated to the maximum potential energy of the spring.

$$
\begin{aligned}
(P E)_{\max } & =\frac{1}{2} \mathrm{~K} x_{1}^{2}=(K E)_{\max }=\left(K E_{\text {mass }}\right)_{\max }+\left(K E_{s}\right)_{\max } \\
\frac{1}{2} \mathrm{~K} x_{1}^{2} & =\frac{1}{2} \omega_{o}^{2} x_{1}^{2}\left(m+\frac{m_{s}}{3}\right) \quad \Rightarrow \quad \omega_{o}=\sqrt{\frac{\mathrm{K}}{m+\frac{m_{s}}{3}}}
\end{aligned}
$$

In the quasi-static approximation, which we have shown in Sect. 2.2.2 requires that $m \gg m_{s}$, the effect of the spring's mass on the frequency of oscillation is to add one-third of the spring's mass to the moving mass, $m$, that is attached to the moving end of the spring and then calculate the resonance frequency using Eq. (2.3). This technique of using the calculation of the kinetic energy of a distribution of masses to determine the natural frequency of a linear vibratory system is known as Rayleigh's method [4]. In Sect. 3.4.3 we will use Rayleigh's method to approximate the natural frequency by integrating both the potential and the kinetic energies and then taking the square-root of their ratio. Rayleigh's method is used throughout this textbook because it can "improve computational ease dramatically while only slightly reducing accuracy" [5].

\subsubsection{Gravitational Offset}

To this point, the effects of gravity on our mass-spring system have been neglected. There has been a tacit assumption that our mass has been constrained to move in only a single direction by some frictionless guide. Mass-spring systems are used as scales for weighing, whether it is a traditional grocer's spring scale, a sport fishing spring scale, or a digital scale which incorporates an elastic load cell and some electronic system for measuring and displaying the deflection caused by the weight of a load.

If our $z$ axis is defined as increasing in the upward direction, then Hooke's law provides the static downward deflection, $\Delta$, from the spring's unloaded equilibrium position, $z_{o}$, as shown at the left in Fig. 2.4: $\Delta=-(m g) / \mathrm{K}$.

The right side of Fig. 2.4 is a plot of the gravitational potential energy, $(P E)_{\text {grav }}=m g\left(z-z_{o}\right)$, represented as the dotted line, and the elastic potential energy, $P E=(1 / 2) \mathrm{K}\left(z-z_{o}\right)^{2}$, represented as the dashed parabola; and their sum is shown as the solid line. The origin of the $z$ axis in that figure has been translated to place the equilibrium position of the unloaded spring at $z_{o}=0$.

The minimum of the sum of those two potential energies is shifted to a negative $z$ value, indicating that the addition of the mass has produced $\Delta<0$. Using the formalism developed in Sect. 1.2, the net 

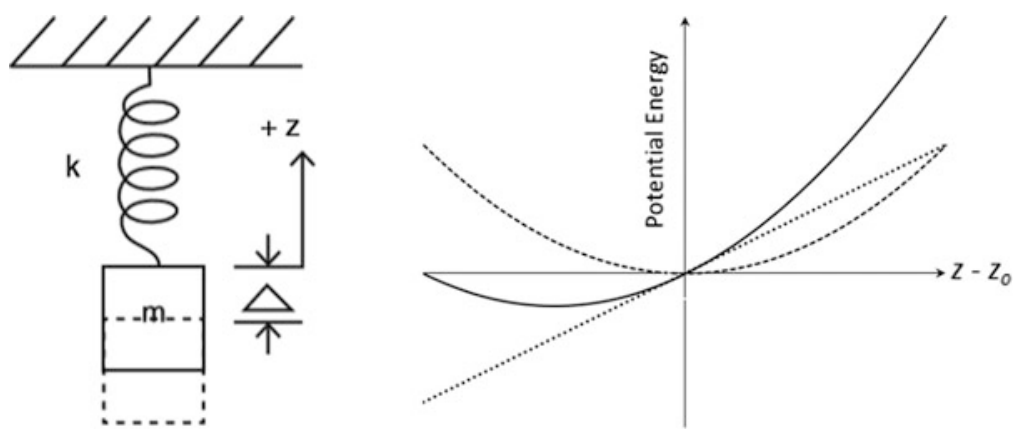

Fig. 2.4 (Left) A spring of equilibrium length, $L$, is suspended from a rigid support, and a mass, $m$, is hung from the spring causing a static deflection, $\Delta$, from its unloaded equilibrium position, $z_{o}$. (Right) The solid line represents the total potential energy on the vertical axis as a function of position, $\left(z-z_{o}\right)$, plotted on the horizontal axis. The dotted line represents the gravitational potential energy, $(P E)_{\text {grav }}=m g\left(z-z_{o}\right)$. The dashed parabola represents the elastic potential energy, $P E=(1 / 2) \mathrm{K}\left(z-z_{o}\right)^{2}$

force at the new position of equilibrium will be given by the first derivative of the potential energy. When the net force is zero, the mass will be at its equilibrium position, $-\Delta$.

$$
\frac{d(P E)}{d z}=-\mathrm{K} z-m g=0 \quad \Rightarrow \quad \Delta=-m g / \mathrm{K}
$$

As shown in Eq. (1.25), the second derivative of the potential energy provides the stiffness, which is unaltered by the displacement produced by the attachment of the weight, $m g$.

$$
\left(\frac{d^{2}(P E)}{d z^{2}}\right)_{z=\Delta}=-\mathrm{K}
$$

The fact that the spring stiffness is unaltered by the displacement produced by the attachment of the weight is a consequence of the linearity of Hooke's law. As long as the weight does not take the spring beyond its linear regime (see Fig. 1.4), the oscillation frequency, $\omega_{o}=(\mathrm{K} / m)^{1 / 2}$, will be the same as in the previous case where we neglected gravity and assumed that the one-dimensional displacements were maintained by some frictionless constraint.

It can be useful to recognize that the natural frequency of the mass-spring system subject to the force of gravity can be expressed in terms of the magnitude of the static displacement, $|\Delta|$, produced when a mass, $m$, is added to the spring of stiffness, K. From Eq. (2.28), we see that $\mathrm{K}=|m g / \Delta|$. Substitution of that result into Eq. (2.3) allows the natural frequency, $\omega_{o}$, to be specified in terms of the gravitational acceleration, $g$, and the magnitude of the static displacement, $\Delta$.

$$
\omega_{o}=\sqrt{\frac{g}{\Delta}}
$$

This form of the result can be convenient for the design and analysis of vibration isolation systems discussed in Sect. 2.5.7.

The pendulum is a simple harmonic oscillator with a natural frequency that is explicitly dependent upon gravity. In this case, there is no "spring" and gravity provides the restoring force. Using the coordinate system of Fig. 1.5, the angular equivalent of Eq. (2.1) is obtained by equating the moment of inertia of the suspended mass, $I=m L^{2}$, times its angular acceleration, $\ddot{\theta}=d^{2} \theta / d t^{2}$, to the angledependent torque, $N(\theta)$, derived in Eq. (1.27). 


$$
\left(m L^{2}\right) \frac{d^{2} \theta}{d t^{2}}+m g L\left[\theta-\frac{\theta^{3}}{6}+\ldots\right]=0
$$

Restricting the angular displacements to small values, $\theta \ll 1$, the nonlinear term in the restoring force can be neglected. If we restrict the maximum deflection to $\theta_{1} \leq 0.10 \mathrm{rad}=5.7^{\circ}$, the ratio of the nonlinear contribution to the linear contribution will be less than $\theta_{1}^{2} / 6=0.17 \%$.

After cancellation of common terms, the expression can be cast into the same form as Eq. (2.1) with $\theta$ as the independent variable and $\omega_{o}{ }^{2}=g / L$.

$$
\ddot{\theta}+\frac{g}{L} \theta=\ddot{\theta}+\omega_{o}^{2} \theta=0 \quad \Rightarrow \quad \omega_{o}=\sqrt{\frac{g}{L}}
$$

Unlike the natural frequency, $f_{o}$, of the mass-spring system, the natural frequency of the pendulum is independent of the mass at the end of the string but is explicitly dependent upon the acceleration due to gravity, $g$.

This is not because the mass is not important to the motion of the pendulum. The disappearance of mass from Eq. (2.32) is a consequence of the fact that the inertia and the restoring force are both directly proportional to the mass, $m$. Since the natural frequency depends upon the ratio of the restoring force (or restoring torque, in this case) to the inertia, the mass cancels out of the expression that provides the natural frequency. It is worthwhile to compare Eq. (2.32) for the frequency of the pendulum to Eq. (2.30) for the frequency of a mass-spring system in a gravitational field. In Eq. (2.30), the explicit dependence on the mass has also disappeared, again due to the fact that the stiffness is being expressed in terms of the static displacement, $\Delta$, caused by the gravitational force on the mass.

\subsubsection{Adiabatic Invariance}

The pendulum provides a simple system that will allow us to demonstrate an additional useful relationship between the energy of a simple harmonic oscillator and its frequency. If we change a constraint on our oscillator, and if we make that change gradually on the time scale set by the period of oscillation, $T$, then the relative change in the energy is equal to the relative change in the natural frequency.

$$
\delta\left(\frac{E}{\omega}\right)=0 \quad \text { or } \quad \frac{\delta E}{E}=\frac{\delta \omega}{\omega}
$$

For slow transitions, ${ }^{3}$ the ratio of the energy to the frequency is a constant. Although this general relationship can be derived from a Lagrangian mechanical perspective [6], it is much better known by its quantum mechanical manifestation as Planck's law for the emission of electromagnetic radiation, $E=\hbar \omega$, where $\hbar \equiv 1.05457182 \times 10^{-34} \mathrm{~J}-\mathrm{s}$ is Planck's constant, $h$, divided by $2 \pi$. In that application, the ratio of energy to frequency is determined by Planck's constant.

\footnotetext{
${ }^{3}$ It is the "slowness" of the transition that gives rise to the "adiabatic" designation. If a transition is abrupt, that is, taking place during much less than one period of oscillation, then energy is deposited in a variety of modes other than the original single mode of oscillation. In our pendulum example, a rapid change in the length of the string attached to the pendulum's mass will launch transverse waves of wavelength, $\lambda<L$, along the string, putting the energy into modes of vibration other than the pendulum mode, as illustrated in Fig. 3.8. (If the length is changed at twice the natural frequency, then it is possible to excite a parametric resonance.) If the change is slow compared to $T$, then the pendulum will continue to swing in the same way but at a frequency that is altered by the slow change in length, $\delta L$.
} 

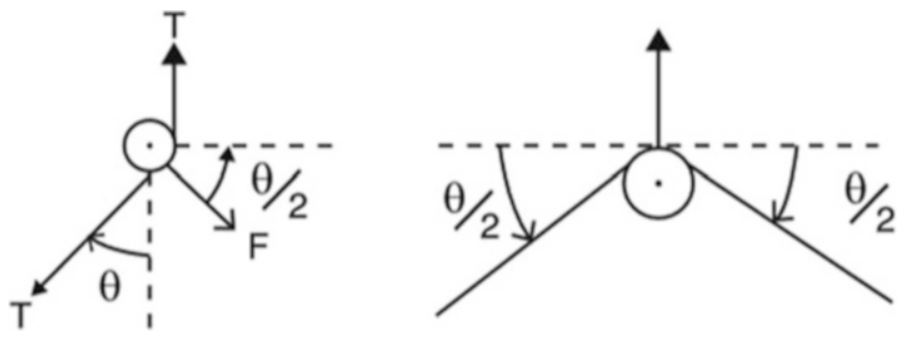

Fig. 2.5 (Left) The support point for the pendulum of Fig. 1.5 is replaced by a pair of frictionless pinch-rollers. Only one roller is shown. The string that supports the pendulum's mass has a tension, T. The left pinch-roller must exert a force $F=2 \mathrm{~T} \sin (\theta / 2)$ on the string to keep it from moving the attachment point. The vertical component of that force is $F_{z}=F$ $\sin (\theta / 2)=2 \mathrm{~T} \sin ^{2}(\theta / 2) \cong \mathrm{T} \theta^{2} / 2$. (Right) This rotated view of the figure on the left simplifies the identification of the angle, $\theta / 2$, used in the diagram of the movable pendulum support. The pinch-rollers must do work against this vertical force to move the attachment point lower and shorten the string

The classical relationship can be applied to the simple pendulum. Logarithmic differentiation of Eq. (2.32) relates the relative change in the pendulum's frequency to the relative change in length by assuming that $g$ is constant.

$$
\frac{\delta \omega_{o}}{\omega_{o}}=-\frac{1}{2} \frac{\delta L}{L}
$$

To demonstrate that this result is consistent with adiabatic invariance, we can imagine that the suspension point for the string that supports the pendulum's mass is squeezed between a pair of frictionless pinch-rollers, as shown in Fig. 2.5. Those rollers can be moved downward to reduce the length of the pendulum by an amount $\delta L$. We will calculate the work required to move the pinch-roller along the string. That should be equal to the amount of energy added to the system by the slow variation of this constraint.

Before calculating that force, it is important to recognize that whether the pendulum moves to the right or to the left, the force on the pinch-rollers is always upward. If we let that force move the pinchrollers, then the pendulum would be doing work on the pinch-rollers, and energy would be removed from the pendulum as the length of the string increases. For the following calculation, we will move the pinch-rollers down, doing work on the pendulum against this force, although either choice would produce a confirmation of adiabatic invariance.

Figure 2.5 shows the force exerted by the pinch-rollers on the string. When the string moves to the left, the pinch-rollers have to exert a force, $F$, that is downward and to the right: $F=2 T \sin (\theta / 2)$. The vertical component of that force in the direction of the motion is $F_{z}=F \sin (\theta / 2)=2 T \sin ^{2}(\theta / 2) \cong T \theta^{2} / 2$.

The tension, $\mathrm{T}$, in the string is due to the weight of the mass, $m g$, and the centripetal acceleration of that mass as it swings to and fro at the natural frequency, $\omega_{o}$, and with an angular displacement amplitude, $\theta_{1}$. Selecting the origin of our time coordinate to make $\theta(t)=\theta_{1} \sin \omega_{o} t$ eliminates the need for a phase, $\phi$, that appears in Eq. (2.6).

$$
\mathrm{T}=m g+m L \dot{\theta}^{2}=m g+m L \omega_{o}^{2} \theta_{1}^{2} \cos ^{2} \omega_{o} t=m g\left(1+\theta_{1}^{2} \cos ^{2} \omega_{o} t\right)
$$

This expression can be integrated to calculate the work done by slowly sliding the pulley down by a distance, $\delta L$. 


$$
\delta E=W=\int_{0}^{\delta L} F_{z} d z=m g \int_{0}^{\delta L}\left(1+\theta_{1}^{2} \cos ^{2} \omega_{o} t\right) \frac{\theta_{1}^{2}}{2} \sin ^{2}\left(\omega_{o} t\right) d z
$$

Since we are moving the pulley slowly by a distance, $\delta L$, over a time corresponding to many cycles of period, $\mathrm{T}=2 \pi / \omega_{o}$, we can take the time-average of the trigonometric functions: $\left\langle\sin ^{2}\left(\omega_{o} t\right)\right\rangle_{t}=$ $\frac{1}{2}$ and $\left\langle\cos ^{2}\left(\omega_{o} t\right) \sin ^{2}\left(\omega_{o} t\right)\right\rangle_{t}=\frac{1}{8}$.

$$
\delta E=\frac{\theta_{1}^{2}}{2} m g\left(\frac{1}{2}+\frac{\theta_{1}^{2}}{8}\right) \delta L \cong \frac{\theta_{1}^{2}}{4} m g(\delta L)
$$

The energy of the pendulum can be written as the maximum kinetic energy which equals the total energy as the pendulum swings through $\theta=0: E(\theta=0)=1 / 2 m v^{2}=1 / 2 m L^{2} \theta_{1}^{2} \omega_{o}^{2}$.

$$
\frac{\delta E}{E}=\frac{\frac{1}{4} m g(\delta L)}{\frac{\theta_{1}^{2}}{2} m L^{2} \omega_{o}^{2}}=\frac{1}{2} \frac{g(\delta L)}{L^{2} \omega_{o}^{2}}=\frac{1}{2} \frac{\delta L}{L}=-\frac{\delta \omega_{o}}{\omega_{o}}
$$

Since we are shortening the pendulum by doing work on it, $\delta L<0$, and this result is identical to our expectation based on Eq. (2.34). Although this result only reconfirms our understanding of the relationship between the pendulum's frequency and its length, as we will see in Sect. 6.2.3 and Sect. 6.2.4, and subsequently in Part II as well, adiabatic invariance will allow us to calculate normal mode frequencies of systems that have complicated geometries in Sect. 13.3.5 or will allow us to determine forces when the frequency changes as system constraints are varied in Sect. 15.4.6.

\subsection{Damping and Free-Decay}

With only a mass and a spring, there is no mechanism by which the vibrational energy can be dissipated. Once set in motion, the mass-spring system has a total energy that is conserved, with the energy being transformed from kinetic to potential forms and vice versa as expressed in Eq. (2.17) throughout each cycle. In principle, the amplitude of oscillation will remain unchanged forever. In this section, we will add a third lumped element that will be able to convert a portion of that energy to heat, thereby reducing the amplitude of the oscillations, until the mass-spring-damper system comes to thermal equilibrium with its environment. As will be demonstrated, all of the energy is initially localized in the kinetic and potential energy of the mass and spring, but the damping element will dissipate this ordered energy, converting it to the disordered form known as heat.

\subsubsection{Viscous Damping and Mechanical Resistance}

Although damping can be introduced through a variety of physical mechanisms (e.g., friction or sound radiation), the most common and useful dissipative element is the viscous damper, represented in Fig. 2.6 as a dashpot. A dashpot is typically envisioned as consisting of a cylinder filled with a viscous fluid in which the motion of a movable vane is resisted by viscous drag, like a spoon being moved slowly through honey. The force of that viscous damping element is proportional to the velocity difference between its ends and is directed opposite to that velocity. Assuming that one end of the dashpot is immobilized, then the force, 


$$
F_{v i s}=-R_{m} v
$$

where $R_{m}[\mathrm{~kg} / \mathrm{s}]$ is called the mechanical resistance. Adding the viscous force to the previous equation, which included inertia and stiffness, produces the equation of motion for a viscously damped simple harmonic oscillator.

$$
m \frac{d^{2} x}{d t^{2}}+R_{m} \frac{d x}{d t}+\mathrm{K} x=0 \quad \text { or } \quad \ddot{x}+\frac{R_{m}}{m} \dot{x}+\omega_{o}^{2} x=0
$$

The solution of this second-order differential equation with constant coefficients highlights the extraordinary utility of complex exponentials. We will postulate that the (complex) solution will have the form $\mathbf{x}(t)=\widehat{\mathbf{C}} e^{\eta t}$, substitute into Eq. (2.39), and determine the form of our (as yet) undetermined exponent, $\eta$.

$$
\left(\eta^{2}+\frac{R_{m}}{m} \eta+\omega_{o}^{2}\right) \widehat{\mathbf{C}} e^{\eta t}=0
$$

To satisfy this equation, the term in parentheses must vanish, since the value of $\widehat{\mathbf{C}} e^{\eta t}$ varies with time. This requirement is met by use of the quadratic formula to specify those two roots corresponding to the two values of $\eta$.

$$
\eta=\frac{-\frac{R}{m} \pm \sqrt{\left(\frac{R_{m}}{m}\right)^{2}-4 \omega_{o}^{2}}}{2}=-\frac{1}{\tau} \pm \sqrt{\left(\frac{1}{\tau}\right)^{2}-\omega_{o}^{2}}
$$

The choice of $\tau=2 \mathrm{~m} / R_{m}$ makes sense since $\tau$ has the units of time, as required for dimensional homogeneity.

As with any new result, it is always valuable to examine the limiting case that corresponds to a previous solution (i.e., no damping). If we let $R_{m}$ go to zero, making $\tau$ go to infinity, we should recover the earlier result in Eq. (2.7).

$$
\lim _{R_{m} \rightarrow 0} \eta= \pm j \omega_{o}
$$

After taking the real part of the assumed exponential solution, $\mathbf{x}=\widehat{\mathbf{C}} e^{\eta t}$, once Eq. (2.41) has been substituted for $\eta$, we see that $\tau$ corresponds to the exponential decay time for the amplitude of the oscillation.

$$
x(t)=\mathfrak{R e}\left[\widehat{\mathbf{C}} e^{\eta t}\right]=|\widehat{\mathbf{C}}| e^{-t / \tau} \cos \left(\omega_{d} t+\phi\right)
$$

The damped natural frequency, $\omega_{d}$, is provided in Eq. (2.45).

Figure 1.18 shows the time evolution of one such damped harmonic oscillator. The motion is no longer strictly periodic. The peak amplitude of each cycle is less than the amplitude of the previous cycle by an amount known as the logarithmic decrement. As in the undamped case, the frequency is still amplitude independent. In the damped case, we can define the frequency as the reciprocal of the time between upward or downward zero-crossings, since zero is not amplitude dependent. Equation (2.41) has also led to the introduction of the damped oscillation frequency, $\omega_{d}$, that will be discussed further in the next section. For the undamped case, where $R_{m}=0, \tau \rightarrow \infty, \omega_{d}=\omega_{o}$. When $R_{m} \neq 0$, the period of the oscillatory portion described by Eq. (2.43) becomes longer. 
Fig. 2.6 A dashpot representing the viscous resistance, $R_{m}$, has been added to the mass and spring system of Fig. 2.4. The dashpot symbol is meant to represent a vane in an oil bath that will provide a viscous force that is proportional and opposite to the velocity of the vane: $F_{v i s}=-R_{m} v$

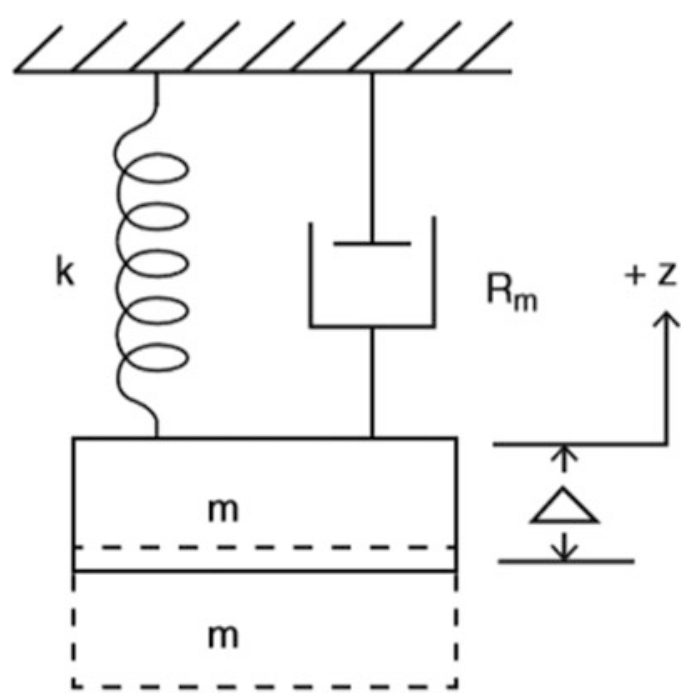

\subsubsection{Free-Decay Frequency and Quality Factor}

In the previous chapter's discussion of similitude (see Sect. 1.7.1), the addition of $R_{m}$ as a third parameter that is required to specify a damped harmonic oscillator introduced a dimensionless group which was labeled $Q^{2}$ at that time in Eq. (1.81), without justification.

$$
Q^{2}=\frac{\mathrm{K} m}{R_{m}^{2}}=\frac{\omega_{o}^{2} m^{2}}{R_{m}^{2}}=\frac{\omega_{o}^{2} \tau^{2}}{4}
$$

Similitude analysis suggests that the square root of this dimensionless group, $Q=\left(\omega_{o} \tau\right) / 2$, known as the quality factor, must provide the only combination of parameters that can control the system's response, even if it is impossible to specify the exact functional form of that control from only dimensional arguments. To see this, we write $\tau=2 Q / \omega_{o}=Q /\left(\pi f_{o}\right)=Q T / \pi$. This shows that $Q$ corresponds to $\pi$ times the number of cycles it takes for the amplitude of a damped harmonic oscillator to decay to $e^{-1} \cong 36.8 \%$ of its initial amplitude. Similarly, substitution into Eq. (2.41) shows that the damped oscillation frequency, $\omega_{d}$, is related to the undamped natural frequency, $\omega_{o}$.

$$
\omega_{d}=2 \pi f_{d}=\sqrt{\omega_{o}^{2}-\left(\frac{1}{\tau}\right)^{2}}=\omega_{o} \sqrt{1-\frac{1}{4 Q^{2}}} \cong \omega_{o}\left(1-\frac{1}{8 Q^{2}}\right)
$$

The expression on the far right is an approximation (using the binomial expansion of Eq. 1.9), which is valid for lightly damped oscillators if $Q^{2} \gg 1$. A related expression can be written for the logarithmic decrement, $\delta$, where $T_{d}=2 \pi / \omega_{d}$ is the period of the damped sinusoid.

$$
\begin{aligned}
& \delta=\ln \left[\frac{e^{-t / \tau}}{e^{-\left(t+T_{d}\right) / \tau}}\right]=\ln \left[e^{-T_{d} / \tau}\right]=-\frac{T_{d}}{\tau}=\frac{2 \pi}{\omega_{d} \tau} \\
& \delta=\frac{\pi}{Q}\left(1-\frac{1}{4 Q^{2}}\right)^{-1 / 2} \cong \frac{\pi}{Q}\left(1+\frac{1}{8 Q^{2}}\right)
\end{aligned}
$$


We can use the parameter values determined by the fit of Eq. (1.116), which is identical in form to Eq. (2.43), to develop some intuition about the results just obtained for a damped harmonic oscillator.

$$
V\left(t_{i}\right)=V_{o} e^{-t_{i} / \tau} \sin \left(2 \pi f_{d} t_{i}+\phi\right)
$$

The fit values provided in the caption of Fig. 1.18 are $f_{d}=46.141 \mathrm{~Hz}$ and $\tau=0.0445 \mathrm{~s}$. Based on Eq. (2.45), let's assume that $\omega_{d} \cong \omega_{o}$ and use the measured $\tau$ to calculate $Q=\left(\omega_{o} \tau\right) / 2=\pi f_{d} \tau=6.45$. Substitution of that result back into Eq. (2.45) suggests that $\omega_{d} / \omega_{o}=0.997$, so treating the natural and damped frequencies as being equal for the initial calculation of the quality factor, $Q$, was a most reasonable choice.

\subsubsection{Critical Damping}

The solution to the quadratic formula for $\eta$ in Eq. (2.41) has three regimes. If $\omega_{o} \tau=2 Q \gg 1$, then the solutions are oscillatory and exhibit an exponentially decaying amplitude with time, as described in Eq. (1.119) and illustrated in Fig. 1.18. Such solutions are called underdamped.

In the opposite limit, the system is overdamped. That means that $R_{m} / \omega_{o} \gg m$ or equivalently $R_{m} / \omega_{o} m=Q^{-1} \gg 1$. In that limit, the behavior of the system is governed by a first-order differential equation, where the inertial term, $m\left(d^{2} x / d t^{2}\right)$, in Eq. (2.39), can be ignored.

$$
R_{m} \frac{d x}{d t}+\mathrm{K} x=0
$$

Being a first-order differential equation, it possesses only one solution corresponding to exponential relaxation.

$$
\int \frac{d x}{x}=-\frac{\mathrm{K}}{R_{m}} \int d t \quad \Rightarrow \quad x(t)=C e^{-\left(\mathrm{K} / R_{m}\right) t}
$$

If the mass is displaced from equilibrium by an amount $x_{1}$, it is dragged back to equilibrium by the spring and $x(t)=x_{1} e^{-t / \tau}$, where $\tau=R_{m} / \mathrm{K}$.

An intermediate case can produce critically damping. If $\omega_{0} \tau_{\text {crit }}=1$ (i.e., $Q=1 / 2$ ), the term under the radical in Eq. (2.41) is zero: $\tau_{c r i t}=\omega_{o}{ }^{-1}=(\mathrm{m} / \mathrm{K})^{1 / 2}$. If the system is initially displaced from equilibrium by an amount $x_{1}$, then the position decays exponentially to zero, like the solution in Eq. (2.48). Critical damping brings the mass back to its equilibrium position in the least amount of time without crossing zero. For the mass to approach the equilibrium position in the minimum time, the damping should be about $60 \%$ of the critical value, although when "critically underdamped," the mass will cross zero before coming to rest.

\subsubsection{Thermal Equilibrium and Fluctuations}

"The phase (or interferometric) sensor, ${ }^{4}$ whether for magnetic, acoustic, rotation, etc., sensing, theoretically
offers orders of magnitude increased sensitivity over existing technologies. In the case of the acoustic sensor

\footnotetext{
${ }^{4}$ The fact that these authors are discussing a fiber-optic interferometric sensor is irrelevant, in this context. Such a sensor uses optical interference to detect changes in the difference of the length of two optical fibers. For our purposes, the interferometric sensor only provides a long "optical lever" like the mirrored galvanometer, shown in Fig. 2.6, that will be analyzed later in this section.
} 
constructed utilizing optical fiber interferometers, these theoretical predictions have been verified to the limit of state of the art in acoustic measurements... In the case of the magnetic sensor, it appears that fiber sensors operating at room temperature offer detection sensitivities comparable to or exceeding cryogenic SQUID technology, which normally operate between 4 and $10 \mathrm{~K}$." [7]

The claims in the above quotation are completely wrong (and cost the US taxpayer millions of dollars as those authors chased this "impossible dream"). That quote epitomizes the problem that is encountered if we neglect the fact that the addition of our resistive element to the mass-spring harmonic oscillator opens a two-way street for the exchange of energy with the environment. Before the inclusion of $R_{m}$, the total energy of the system was determined by the initial conditions and that total energy remained constant, although the energy shuttled back and forth between its kinetic and potential forms. With a dissipative element, there was a path for energy to leave the oscillator, in this case as heat. ${ }^{5}$ That path also connects the oscillator to "the environment" that must share energy with the oscillator by virtue of the fact that the absolute (kelvin) temperature of the environment and $R_{m}$ is both non-zero. Temperature is a measure of the average kinetic energy of the particles that make up matter. Given sufficient time, every degree of freedom will get its "fair share." This result is known as the Equipartition Theorem and can be thought of as a definition of thermal equilibrium, being the condition where, on average, each degree of freedom has the same average energy.

For degrees of freedom that have energies that are quadratic in their position or momentum coordinates, ${ }^{6}$ that "fair share" is (1/2) $k_{B} T$, where the Boltzmann's constant is $k_{B} \equiv 1.380649 \times 10^{-23} \mathrm{~J} / \mathrm{K}$ and $T$ is the absolute (kelvin) temperature.

Before examining the consequences of thermal equilibrium on the limiting behavior of a damped harmonic oscillator due to thermal fluctuations, it may provide some comfort to reflect on the history of science, since it demonstrates that our understanding of heat and temperature took a relatively long time to develop. By comparison, our classical understanding of gravity and dynamics of particles from Newton's falling apple to the orbits of the planets in the solar system, seemingly a far more arcane problem, was completely understood two centuries before we had a fundamental understanding of temperature and the mechanical equivalence of heat.

The year 1642 was an important milestone on the path to our current scientific understanding of the physical world. Galileo died in January of that year and Newton was born on December 25 of the same year, which turned out to be our guarantee that the scientific method would survive the death of Galileo. ${ }^{8}$ Kepler (1571-1630) published his first two laws of planetary motion in 1609 and his third (see Sect. 2.3.1) in 1619, based on the observations of Tycho Brahe (1546-1601). Newton's Philosophice Naturalis Principia Mathematica was first published in 1687. It unified the dynamics of falling bodies on earth with the orbital motion of the planets in our solar system through Newton's laws of motion and his law of universal gravitation.

\footnotetext{
${ }^{5}$ As will be shown in Chap. 12, energy can also leave by radiation of sound, but the thermodynamic consequences of energy being shed to the environment by any means are identical.

${ }^{6}$ The potential energy of a spring, $(1 / 2) \mathrm{K} x^{2}$, is quadratic in displacement. Kinetic energy, $(1 / 2) m v^{2}=(1 / 2) p^{2} / m$, is quadratic in the momentum, $p$. For degrees of freedom that have energies that are quartic in the generalized coordinate, each degree of freedom gets $(1 / 4) k_{B} T$ in thermal equilibrium.

${ }^{7}$ The most accurate value of Boltzmann's constant is determined by a sound speed measurement, e.g., M. de Podesta et al., "A low-uncertainty measurement of the Boltzmann constant," Metrologia 50, 354-376 (2013). As of 20 May 2019 , the value of $k_{B}$ that is used here is taken to be exact (see Appendix A), and other practical units, such as the kilogram, the second, the meter, and the kelvin, are defined in terms of those "exact" fundamental physical constants.

${ }^{8}$ During the winter holiday season, some godless Humanists (commonly called atheists) celebrate "Continuity Day" to commemorate the continuity of the scientific method.
} 
One would think that the concepts of heat and temperature, which are familiar to us through our own senses, would have been understood much earlier, but exactly the opposite is true. James Prescott Joule (1818-1889) measured the mechanical equivalence of heat (now incorporated into the First Law of Thermodynamics) in $1845,{ }^{9}$ nearly 160 years later than Newton's Principia. In fact, the connection between molecular motion and temperature was not established until the work of James Clerk Maxwell (1831-1879) and Ludwig Boltzmann (1844-1906) introduced the kinetic theory of gases and statistical mechanics (see Chaps. 7 and 9). Boltzmann went to his grave defending his theory against opposition within the scientific community at that time, and his gravestone has $S=k \ln W$ at its top, where $S$ is entropy, $k=k_{B}$ is Boltzmann's constant, and $W$ (for Wahrscheinlichkeit) is the number of microscopic states (i.e., combinations of positions and velocities) that correspond to the macroscopic equilibrium state of the system.

The first recorded observation of the mechanical consequences of thermal fluctuations was the microscopic observation in 1827 by botanist Robert Brown (1773-1858) of the motion of pollen particles suspended in water. ${ }^{10}$ The theoretical explanation for this random motion was published by Albert Einstein (1879-1955) in 1905 [8] and was the reason, along with the photoelectric effect, that he was awarded the Nobel Prize in 1921. ${ }^{11}$ The generalization of the role of thermal fluctuation to mechanical and electrical dissipation did not really start to develop until the 1920s. Most relevant to our immediate interests are thermal fluctuations in simple harmonic oscillators, which started with the observation of random motion in the mirrored galvanometer.

The mirrored galvanometer was patented by William Thompson (Lord Kelvin) in $1858 .{ }^{12}$ It consists of a coil of wire, placed in a magnetic field, acting as an electrical meter that uses a mirror to reflect a beam of light to a scale, usually several meters away on a wall. That long "optical lever" provided a sensitive indication of the current passing through the coil. A torsion fiber provided the restoring force for the mirror-coil combination. (A similar arrangement is shown in Fig. 15.33.)

It was noticed that the spot of light reflected from the mirror underwent random motion. At first, it was assumed that the random motions were due to the collision of air molecules with the mirror, and a study was undertaken to measure the fluctuating position of the light spot as air was pumped out of the galvanometer [9]. Even without air, the random motions were still observed. In 1929, Uhlenbeck and Goudsmit were able to show that those fluctuations "depend on the properties of the observed system and on the temperature only" 13 [10]. At the same time, Nyquist was able to demonstrate that electrical

\footnotetext{
${ }^{9}$ That was the date when he presented his results at a scientific meeting. Those results were not published until 1850: J. P. Joule, "On the mechanical equivalent of heat," Phil. Trans. Roy. Soc. 140(Part I), 61-82 (1850).

${ }^{10}$ The fact that such motions were not biological in origin was confirmed when similar motion was observed in a drop of water trapped in amber (i.e., fossilized tree sap). No living creature could have survived that long in a water droplet isolated since antiquity.

${ }^{11}$ Einstein's Nobel Prize in Physics did not mention his theory of relativity since it was still too controversial in 1921.

${ }^{12}$ Thompson played a significant role in the laying of the first transatlantic telegraph cable between Ireland and Newfoundland that was successfully completed in 1865. He traveled on the cable-laying ship, the Great Eastern, as scientific advisor for all electrical matters. The mirrored galvanometer allowed detection of the weak electrical signals that made it through the cable. He set up two companies to sell these galvanometers and provide engineering consulting services to submarine cable companies. Those endeavors made him a wealthy man. The fascinating story of this first transatlantic cable is told by J. S. Gordon in A Thread Across the Ocean (Perennial, 2003): ISBN 978-0060524463.

${ }^{13}$ It is worth mentioning that these two Dutch physicists were also responsible for postulating that the electron had an internal degree of freedom that became known as the "electron spin."
} 

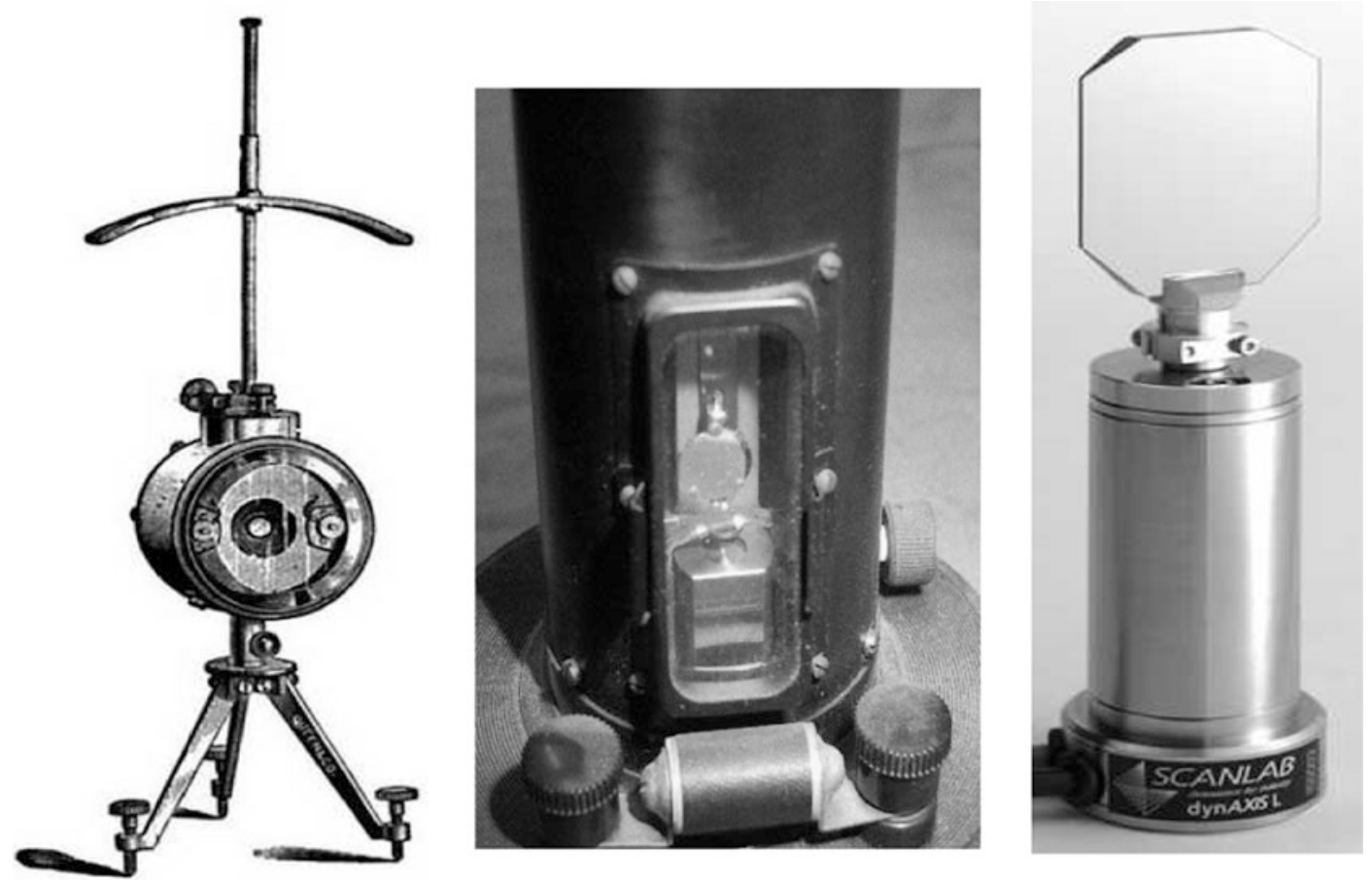

Fig. 2.7 (Left) Drawing of Lord Kelvin's patented mirrored galvanometer. (Center) Photograph of a mirrored galvanometer from the author's collection. (Right) Although such instruments are no longer used to measure electrical currents, the same type of device is used to deflect laser beams for optical scanning purposes. (Photo courtesy of Scanlab, http://www.scanlab.de)

noise was generated by electrical resistors (Johnson noise [11]) based on their temperature [12] (Fig. 2.7).

Again, this very long historical digression is intended to point out that the connection between temperature and thermal fluctuations took a very long time before it was understood, along with its consequences for setting a minimum detectable signal for sensors [13]. The absurdity of the quote that started this section by claiming that "fiber sensors operating at room temperature offer detection sensitivities comparable to or exceeding cryogenic SQUID technology, which normally operate between 4 and $10 \mathrm{~K}$ " [7] is simply a reflection (no pun intended) of those authors' ignorance of the fluctuations that had been observed in the 1920s with mirrored galvanometers [14]. In effect, their "optical lever" was 1-1000 m of optical fiber. The first fiber-optic interferometric sensor that was limited by thermal fluctuations was demonstrated experimentally in 1987 [15].

The Equipartition Theorem contradicts the result in Eq. (2.43), which claims that the amplitude of the motion of the mass connected to the spring and damper will decay to as small a value as you like, if you are willing to wait long enough for the exponential factor, $|\widehat{\mathbf{C}}| e^{-t / \tau}$, to reduce the oscillatory amplitude. In thermal equilibrium, the mean value of each independent quadratic term in the energy will contain an average amount of energy that is $(1 / 2) k_{B} T[16]$.

This result can be applied to the kinetic energy of the pollen particle that Brown observed jittering under his microscope in 1827. Today, it is easier to observe the motion of colloidal gold suspensions that are made with particle diameters of $5 \times 10^{-6} \mathrm{~cm}$ [17]. The mass of each gold particle is about $10^{-15} \mathrm{~g}$. If we equate their kinetic energy per degree of freedom to $(1 / 2) k_{B} T$, and account for the fact that the 
particles move in three dimensions (hence, there are three quadratic degrees of freedom corresponding to the velocities $v_{x}, v_{y}$, and $v_{z}$ ), we can solve for the gold particles' root-mean-squared velocity, $v_{r m s}$.

$$
v_{r m s}=\left\langle v^{2}\right\rangle^{1 / 2}=\left\langle v_{x}^{2}+v_{y}^{2}+v_{z}^{2}\right\rangle^{1 / 2}=\sqrt{\frac{3 k_{B} T}{m}}
$$

For such a colloidal suspension at $T=300 \mathrm{~K}, v_{r m s} \cong 10 \mathrm{~cm} / \mathrm{s}$, which is what is observed [17]. If we make the same calculation for a $1 \mathrm{~g}$ sphere at $300 \mathrm{~K}, v_{r m s} \cong 3.5 \times 10^{-9} \mathrm{~m} / \mathrm{s}=11 \mathrm{~cm} /$ year, which is why we don't see such small (but macroscopic) particles moving about randomly. ${ }^{14}$

The same approach can be applied to our single degree-of-freedom mass-spring-damper system to calculate the steady-state motion of the mass by equating the thermal energy per degree of freedom ( $1 / 2)$ $k_{B} T$ to the potential energy, $(1 / 2) \mathrm{K} x^{2}$.

$$
x_{r m s}=\left\langle x^{2}\right\rangle^{1 / 2}=\sqrt{\frac{k_{B} T}{\mathrm{~K}}}
$$

For the mirrored galvanometer, we would get the same result for $\theta_{r m s}$ except that $\mathrm{K}$ would be the torsional stiffness instead of the linear stiffness. Again, for macroscopic masses and ordinary temperatures, the fluctuations in the position of the mass are insignificant, but with the recent ubiquity of micro-electro-mechanical sensors (MEMS) (e.g., accelerometers and microphones), thermal fluctuations can be the dominant noise source [18] (Fig. 2.8).

Fig. 2.8 The decay of a mass-spring system due to damping given by the time history in Eq. (2.43) is only true for displacements that are substantially larger than the thermal fluctuation amplitude, $x_{r m s}$, given in Eq. (2.50). In reality, the motion of the mass does not decay to zero but will fluctuate randomly about its equilibrium position indefinitely due to its thermal energy. (Diagram courtesy of T. B.

Gabrielson)

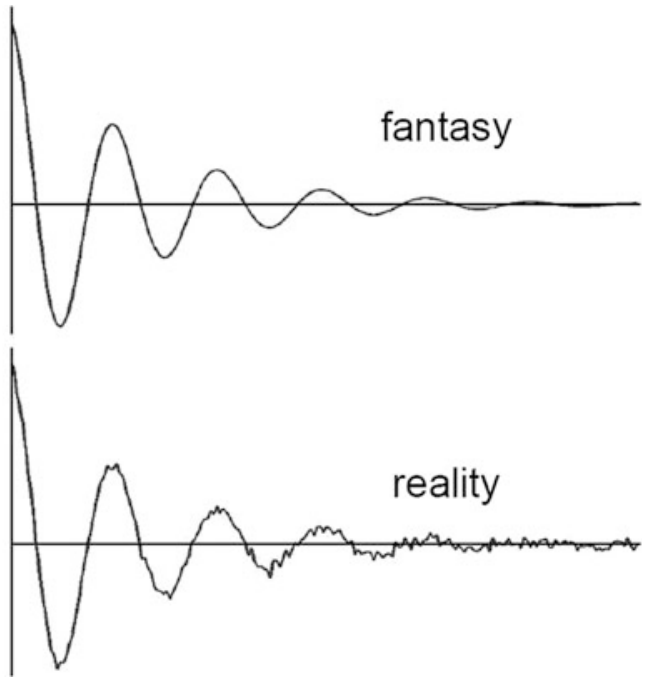

\footnotetext{
${ }^{14}$ The precise measurements of Brownian motion that were used to test Einstein's theory were made by Harvey Fletcher (1884-1981) with oil droplets as part of his Ph.D. thesis under the supervision of R. A. Millikan. His advisor chose to publish the charge quantization demonstrated by that oil drop experiment under his own name and received the Nobel Prize in Physics in 1923. Fletcher published the Brownian motion measurements under his name and went on to a very successful career in acoustics at Bell Labs: M. F. Perry, "Remembering the oil-drop experiment," Phys. Today 60(5), 56-60 (2007).
} 
Fig. 2.9 The transfer function, $H(\Omega)$, of a singlestage low-pass filter (solid line) is plotted against the normalized frequency, $\Omega=f l f_{-3 \mathrm{~dB}}$, where $f_{-3 \mathrm{~dB}}$ is the frequency at which the amplitude of the transfer function has decreased to $\sqrt{2}$ of its low-frequency limit. The dashed rectangle has the value one up to $\Omega=\pi / 2$ and zero for $\Omega>\pi /$ 2 , giving it the same area as that under the solid line. For that reason, $(\Delta f)_{E Q N B}=$ $\pi f_{-3 \mathrm{~dB}} / 2$

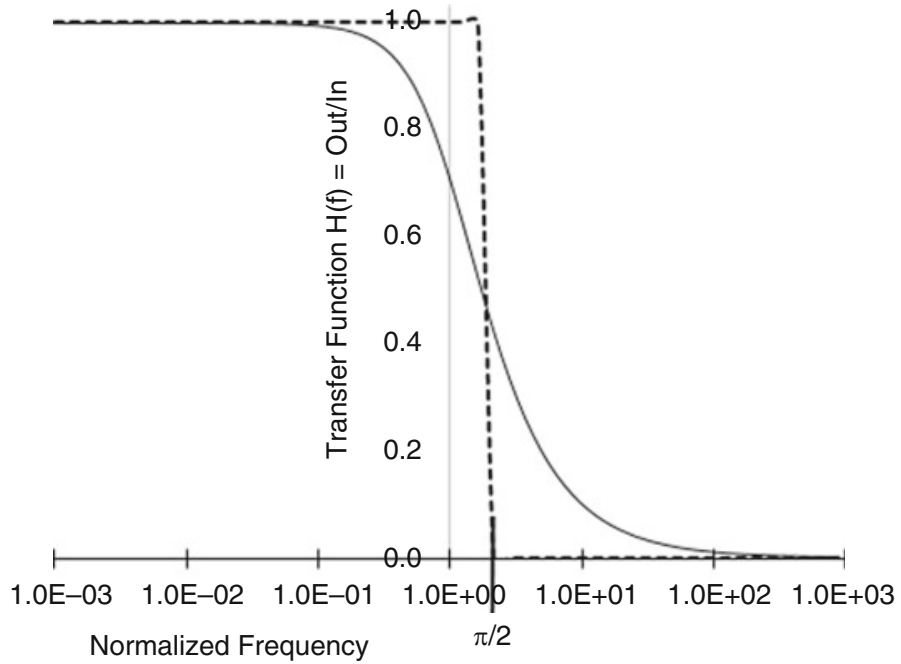

This random motion can be interpreted as the response to a fluctuating force, $F_{\text {noise }}$. Its root-meansquared value is related to the square root of the product of the absolute (kelvin) temperature, $T$; the mechanical resistance, $R_{m}$; and the equivalent noise bandwidth, $(\Delta f)_{E Q N B}$, of the system.

$$
F_{r m s}=\left\langle F_{n o i s e}^{2}\right\rangle^{1 / 2}=\sqrt{4 k_{B} T R_{m}(\Delta f)_{E Q N B}}
$$

The part of this expression which may be unfamiliar at this point is $(\Delta f)_{E Q N B}$. At the simplest level, that bandwidth specifies the range of frequencies over which the fluctuating force will preferentially excite the system's response. The "equivalence" is a way to hide the fact that one needs to integrate over the entire system's frequency response function then choose an equivalent "rectangular" filter response function that has a frequency width of $(\Delta f)_{E Q N B}$ and unity gain, as shown in Fig. 2.9. We will calculate this effective bandwidth for a damped driven harmonic oscillator in Sect. 2.5.

At this point, the attentive reader (hopefully, you!) might become a little anxious. (S)he might reflect thusly: "This section started off claiming that the addition of a mechanical resistance opened a 'two-way street' that let ordered vibrational energy leave the system but also created a path for the random thermal motion to enter the system. I can see in Eq. (2.51) that the fluctuating force is related to that mechanical resistance, but the results for $x_{r m s}=\left\langle x^{2}\right\rangle_{t}^{\frac{1}{2}}=\sqrt{k_{B} T / 2 \mathrm{~K}}$ and $v_{r m s}=\left\langle v^{2}\right\rangle_{t}^{\frac{1}{2}}=$ $\sqrt{k_{B} T / 2 m}$ do not involve the mechanical resistance at all. What gives?"

The lazy textbook author would provide the following response: "Do you remember our calculation of the frequency of the pendulum? The result, $\omega_{o}{ }^{2}=g / L$, does not involve the mass, yet it is the mass of the pendulum that provided the inertia and the mass of the pendulum that was proportional to the gravitational restoring force. In the final result for frequency, which depends upon the ratio of the restoring force to the inertia, the mass cancelled out. The same is true here, though at this point it is not at all obvious. For a simple harmonic oscillator, $(\Delta f)_{E Q N B} \propto R_{m} / m$, so $R_{m}$ comes out of the square-root in Eq. (2.51). At the frequency of peak response, $\omega_{o}$, the velocity of the mass is proportional to $F / R_{m}$, thus cancelling $R_{m}$ out of the final result for $v_{r m s}$. A similar argument holds for $x_{r m s} . "$

The thermal noise of any mechanical system that is in thermal equilibrium with its surroundings, no matter how complex, can be analyzed by including this fluctuating force combined with the resistance; 
just as in electrical circuits, a fluctuating voltage source, $V_{\text {noise }}$, can be included with the electrical resistance, $R_{e l}[11]$.

$$
V_{r m s}=\left\langle V_{\text {noise }}^{2}\right\rangle^{1 / 2}=\sqrt{4 k_{B} T \mathrm{R}_{\mathrm{el}}(\Delta f)_{E Q N B}}
$$

That expression is the "Johnson noise" [11] that is familiar to electrical engineers.

\subsubsection{Frictional (Coulomb) Damping*}

Most of us are first exposed to friction in high school physics classes where the frictional force, $F_{d}$, is the sliding friction between two dry surfaces. That frictional force is characterized by a coefficient of sliding friction, $\mu$, assumed to be velocity independent and proportional to the force (usually gravitational) that is squeezing the two surfaces together.

$$
F_{d}=-\mu m g
$$

To determine the amplitude decay for frictional damping, we can calculate the work done by the frictional force during one half-cycle and equate that to the change in the potential energy. Figure 2.10 shows the first few cycles that will be used to calculate the amplitude decrease for successive oscillations.

Starting with an initial displacement of $x_{1}=x(t=0)$, the mass will come to rest instantaneously at the other extreme of the motion, $x_{-1}=x(T / 2)$, where it changes direction. Since there will be frictional losses, $\left|x_{1}\right|>\left|x_{-1}\right|$.

$$
\frac{1}{2} \mathrm{~K}\left(x_{1}^{2}-x_{-1}^{2}\right)=\frac{1}{2} \mathrm{~K}\left(x_{1}-x_{-1}\right)\left(x_{1}+x_{-1}\right)=W_{d}=F_{d}\left(x_{1}+x_{-1}\right)
$$

This results in an expression for the amplitude decrease for the first half-cycle: $\left(x_{1}-x_{-1}\right)=2 F_{d} / \mathrm{K}$. Repeating the procedure for the next half-cycle, we again see that the amplitude is decreased by the same amount resulting in a loss of $\Delta x(T)=x_{1}-x_{2}=4 F_{d} / \mathrm{K}$. This will continue, as illustrated in

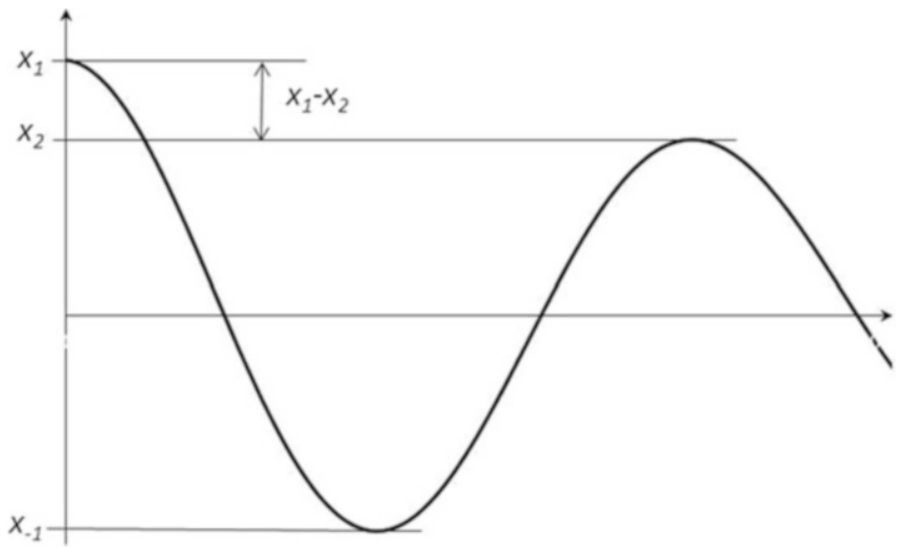

Fig. 2.10 Decay of the amplitude of a simple harmonic oscillator that is subject to sliding friction can be calculated by examination of the losses that occur during each half-cycle. The time history at the right starts with a positive displacement, $x_{1}$, at time, $t=0$, and at the end of the first half-cycle the mass has moved to $x_{-1}$. The energy dissipation guarantees $\left|x_{1}\right|>\left|x_{-1}\right|$. During the second half-cycle, the mass moves from $x_{-1}$ to $x_{2}$, with $\left|x_{-1}\right|>\left|x_{2}\right|$, etc. 
Fig. 2.11 (Left) A viscous damping force, $F_{v i s}=-R_{m} v$, produces an exponentially decaying amplitude. (Right) A frictional (Coulomb) damping force, $F_{d}=-\mu$ $(m g)$, produces an amplitude that decays linearly with time. (Figures from Pippard [19])
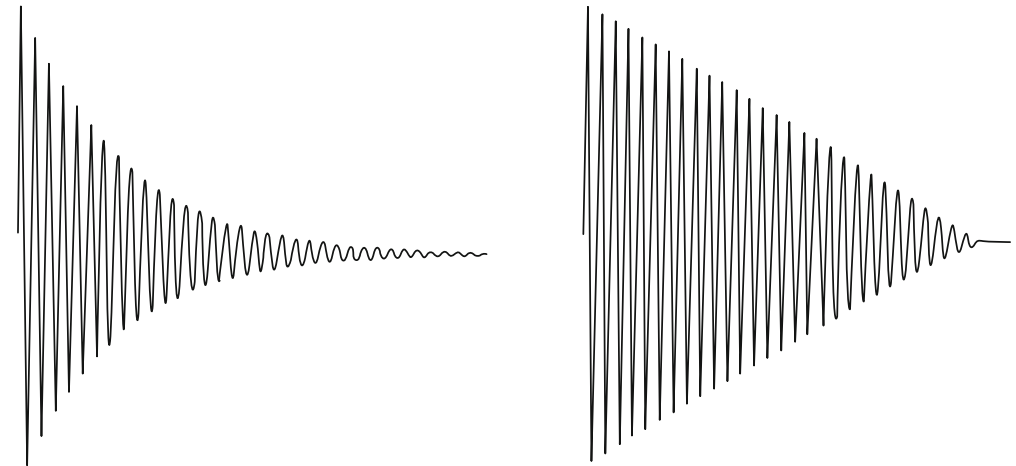

Fig. 2.11, until the mass comes to rest. The decay per cycle is a constant, so the amplitude decreases linearly with time, not exponentially with time [19].

\subsection{Driven Systems}

Thus far, we have only analyzed the response of a harmonic oscillator that is provided with an initial displacement, an initial velocity, or both. Those solutions, like that shown in Eq. (2.43), are allowed to evolve in time without any further energy input. We have also demonstrated that the imposition of a steady gravitational force will displace the equilibrium position but not influence the oscillator's dynamical response. In this section, we will examine the response of a viscously damped simple harmonic oscillator to a periodic force applied to the mass or to a periodic displacement applied to the end of the spring not attached to the mass. The steady-state solution that supplies the response to both of those driven systems will be provided through the calculation of a mechanical impedance. Also, important will be the concept of resonance. When the drive occurs at the natural frequency, $\omega_{o}$, of the undriven system, the steady-state (velocity) response at resonance is only limited by the damping. Resonance is one of the most valuable tools available to the acoustician, and this section will provide our first exposure to its wonders.

\subsubsection{Force-Driven SHO}

We start by assuming that a sinusoidally varying force of amplitude, $F_{1}$, is applied to the mass in a mass-spring-damper system. The force amplitude, $F_{1}$, can be chosen to be a real number without any loss of generality since that choice indicates that any phase shifts will be referenced to the phase of the force, which has now been chosen to define the origin of our phase reference (i.e., $\phi=0$ ), so $F$ $(t)=F_{1} \cos (\omega t)$. When that sinusoidally time-varying force is applied to the mass, then Eq. (2.39) is no longer homogeneous.

$$
m \frac{d^{2} x}{d t^{2}}+R_{m} \frac{d x}{d t}+\mathrm{K} x=m \frac{d v}{d t}+R_{m} v+\mathrm{K} \int v d t=F(t) \equiv F_{1} e^{j \omega t}
$$

Instead of writing our differential equation in terms of the displacement of the mass, it is entirely equivalent to write it in terms of the velocity of the mass.

Although the above equation assumes a force is applied to the mass-spring-damper system at a single driving frequency, $\omega$, our discussion of Fourier synthesis (see Sect. 1.4) indicated that, for such a 
linear system (see Sect. 1.3), we could produce the response to a more complicated periodic driving force by superposition of forces that are applied with other frequencies, amplitudes, and phases.

It is also important to remember that a driven linear system can only respond at the driving frequency, once any transient behavior dies out (see Sect. 2.5.4). Since Eq. (2.39) is homogeneous, solutions to the homogeneous equation, like that in Eq. (2.43), can be added to the solution of Eq. (2.55) in amounts determined by the initial conditions. Because $\tau<\infty$, those transient solutions will eventually die out leaving only the steady-state response.

Again, convenience (complex) numbers (see Sect. 1.5) make the solution to Eq. (2.55) straightforward. Since we know that the linear system's steady-state response occurs only at the driving frequency, $\omega$, we will postulate a solution for the velocity of the mass's response of the form, $v(t)=$ $\mathfrak{R e}\left[\widehat{\mathbf{C}} e^{j \omega t}\right]$, with the understanding that $\mathbf{C}$ is a phasor (complex number) that can introduce a phase shift between the applied force and the velocity, as well as determine the amplitude of the response. We again revert to phasor notation that allows us to combine both the amplitude and phase into a single complex symbol, $\widehat{\mathbf{v}} e^{j \omega t}=\widehat{\mathbf{C}} e^{j \omega t}$, and substitute that solution into Eq. (2.55) and factor out the common term.

$$
\left(j \omega m+R_{m}+\frac{\mathrm{K}}{j \omega}\right) \widehat{\mathbf{C}} e^{j \omega t}=F_{1} e^{j \omega t}
$$

The amplitude and the phase of the velocity response, as represented by the velocity phasor, $\mathbf{v}$, to the applied force of amplitude, $F_{1}$, at frequency, $\omega$, can be written directly.

$$
\widehat{\mathbf{v}} e^{j \omega t}=\frac{F_{1} e^{j \omega t}}{j\left(\omega m-\frac{\mathrm{K}}{\omega}\right)+R_{m}}
$$

The entire response of the driven system is characterized by the complex denominator. By analogy with electrical circuit theory, that denominator is identified as the complex mechanical impedance, $\boldsymbol{Z}_{\boldsymbol{m}}$.

$$
\mathbf{Z}_{\mathbf{m}} \equiv \frac{F}{\widehat{\mathbf{v}}}=j\left(\omega m-\frac{\mathrm{K}}{\omega}\right)+R_{m}
$$

The imaginary component of the mechanical impedance is $\mathfrak{J} m\left[\mathbf{Z}_{\mathbf{m}}\right] \equiv X_{m}=\omega m-(\mathrm{K} / \omega)$. It is called the mechanical reactance and represents the storage of energy as either elastic stiffness or inertia. It is important to recognize that the reactance vanishes, $X_{m}\left(\omega_{o}\right)=0$, when the forcing frequency equals the natural frequency, $\omega=\omega_{o}=\sqrt{\mathrm{K} / m}$.

We recognized $\mathfrak{R e}\left[\boldsymbol{Z}_{\boldsymbol{m}}\right]=R_{m}$ as the mechanical resistance that represents the dissipation of energy. At resonance, when $X_{m}\left(\omega_{o}\right)=0$, the response is controlled entirely by the resistance; hence it is referred to as the resistance-controlled response regime. The steady-state solution for the displacement, $x(t)$; the velocity, $v(t)$; or the acceleration, $a(t)$, in response to the driving force, $F(t)$, can be written compactly in terms of $\mathbf{Z}_{\mathbf{m}}$. The transient response is included in Eq. (2.80).

$$
x(t)=\mathfrak{R} \mathrm{e}\left[\frac{1}{j \omega} \frac{F_{1} e^{j \omega t}}{\mathbf{Z}_{\mathbf{m}}}\right], \quad v(t)=\mathfrak{R e}\left[\frac{F_{1} e^{j \omega t}}{\mathbf{Z}_{\mathbf{m}}}\right], \quad \text { and } a(t)=\mathfrak{R e}\left[j \omega \frac{F_{1} e^{j \omega t}}{\mathbf{Z}_{\mathbf{m}}}\right]
$$

As with any new result, it is prudent to interpret the consequences and compare them to our assumptions. The fact that the mechanical impedance has singled out a specific frequency, $\omega_{o}=$ $\sqrt{\mathrm{K} / m}$, makes it easy to define what is meant by the low- and high-frequency limits. In the low-frequency limit, $\omega \ll \omega_{o}$, the stiffness is dominant, and substitution of Eq. (2.58) into the above 


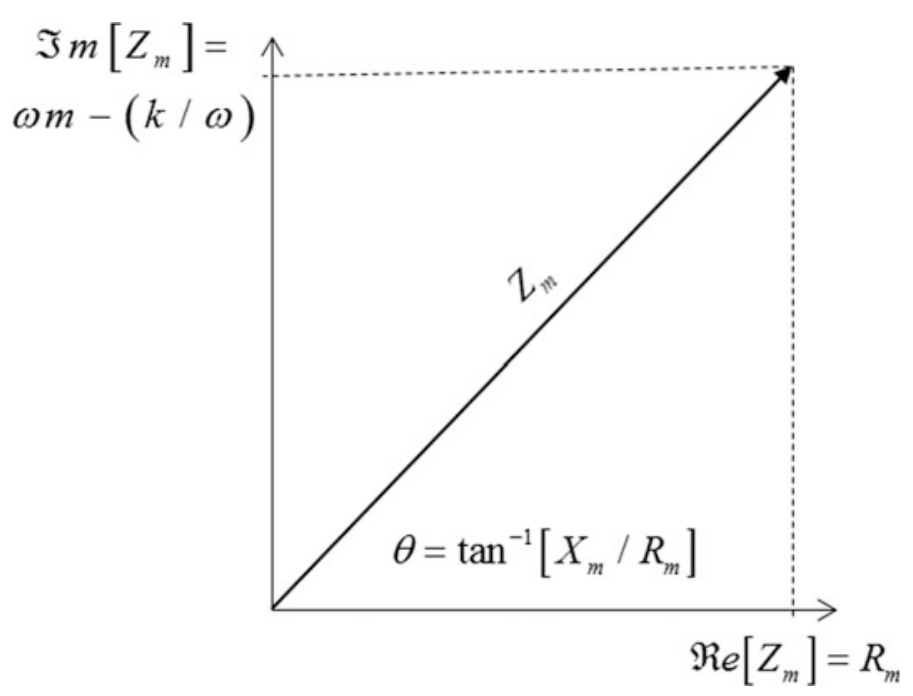

Fig. 2.12 The complex mechanical impedance can be represented in polar form with the frequency-independent real component, $\mathfrak{R e}\left[\boldsymbol{Z}_{\boldsymbol{m}}\right]=R_{m}$, and the imaginary component, $\mathfrak{I m}\left[\boldsymbol{Z}_{\boldsymbol{m}}\right]=X_{m}=\omega m-(\mathrm{K} / \omega)$. The magnitude of the mechanical impedance, $\left|Z_{m}\right|$, is given by the length of the hypotenuse, and the phase angle between the force and the velocity is $\Theta=\tan ^{-1}\left[X_{m} / R_{m}\right]$. That phase angle can vary between the "stiffness-controlled limit," $-90^{\circ}$, well below the resonance frequency (i.e., displacement $x(t)=v(t) /(j \omega)$ out-of-phase with force), and $+90^{\circ}$, the "mass-controlled limit," well above the resonance frequency (i.e., acceleration, $a(t)=(j \omega) v(t)$, is in-phase with the force)

expression, $x(t) \cong-F_{1} / \mathrm{K}$, results in the cancellation of the frequency and the recovery of Hooke's law. For that reason, the low-frequency limit is referred to as the stiffness-controlled response regime.

In this high-frequency limit, $\omega \gg \omega_{o}$, the $j \omega m$ term in the mechanical impedance is dominant, so $a(t) \cong F_{1} / m$ is frequency-independent, and Newton's Second Law is recovered. For that reason, the high-frequency limit is referred to as the mass-controlled response regime.

It is useful to have a polar representation of the mechanical impedance magnitude and phase: $\left|\mathbf{Z}_{\mathbf{m}}\right|=\left(\mathbf{Z}_{\mathbf{m}} \mathbf{Z}_{\mathbf{m}} *\right)^{1 / 2}$ and $\Theta=\arctan \left(X_{m} / R_{m}\right)$. The geometric interpretation is provided in Fig. 2.12.

$$
\begin{gathered}
\left|\mathbf{Z}_{\mathbf{m}}\right|=\sqrt{\mathbf{Z}_{\mathbf{m}} \mathbf{Z}_{\mathbf{m}}^{*}}=\left[R_{m}^{2}+\left(\omega m-\frac{\mathrm{K}}{\omega}\right)^{2}\right]^{1 / 2}=\omega_{o} m\left[\left(\frac{\omega}{\omega_{o}}-\frac{\omega_{o}}{\omega}\right)^{2}+\frac{1}{Q^{2}}\right]^{1 / 2} \\
\Theta=\tan ^{-1}\left(\frac{X_{m}}{R_{m}}\right)=\tan ^{-1}\left[\frac{\omega m-\frac{\mathrm{K}}{\omega}}{R_{m}}\right]=\tan ^{-1}\left[Q\left(\frac{\omega}{\omega_{o}}-\frac{\omega_{o}}{\omega}\right)\right]
\end{gathered}
$$

Figure 2.13 shows the magnitude and phase of the velocity response to the sinusoidal force, $F(t)=F_{1} e^{j \omega t}$. The complex velocity response can be written in a more symmetric form by using $Q=\omega_{o} m / R_{m}$ to substitute for $R_{m}$ in Eq. (2.57).

$$
\mathbf{v}(t)=\frac{F_{1} e^{j(\omega t-\Theta)}}{\left|\mathbf{Z}_{\mathbf{m}}\right|}=\frac{\frac{F}{R_{m} Q} e^{j(\omega t-\Theta)}}{\left[\left(\frac{\omega}{\omega_{o}}-\frac{\omega_{o}}{\omega}\right)^{2}+\frac{1}{Q^{2}}\right]^{1 / 2}}=v_{1}(\omega) e^{j(\omega t-\Theta)}
$$

Expressions with the form of Eq. (2.62) show up for all driven resonance behavior, not just the driven simple harmonic oscillator. As will become apparent throughout this book, this Rayleigh line 


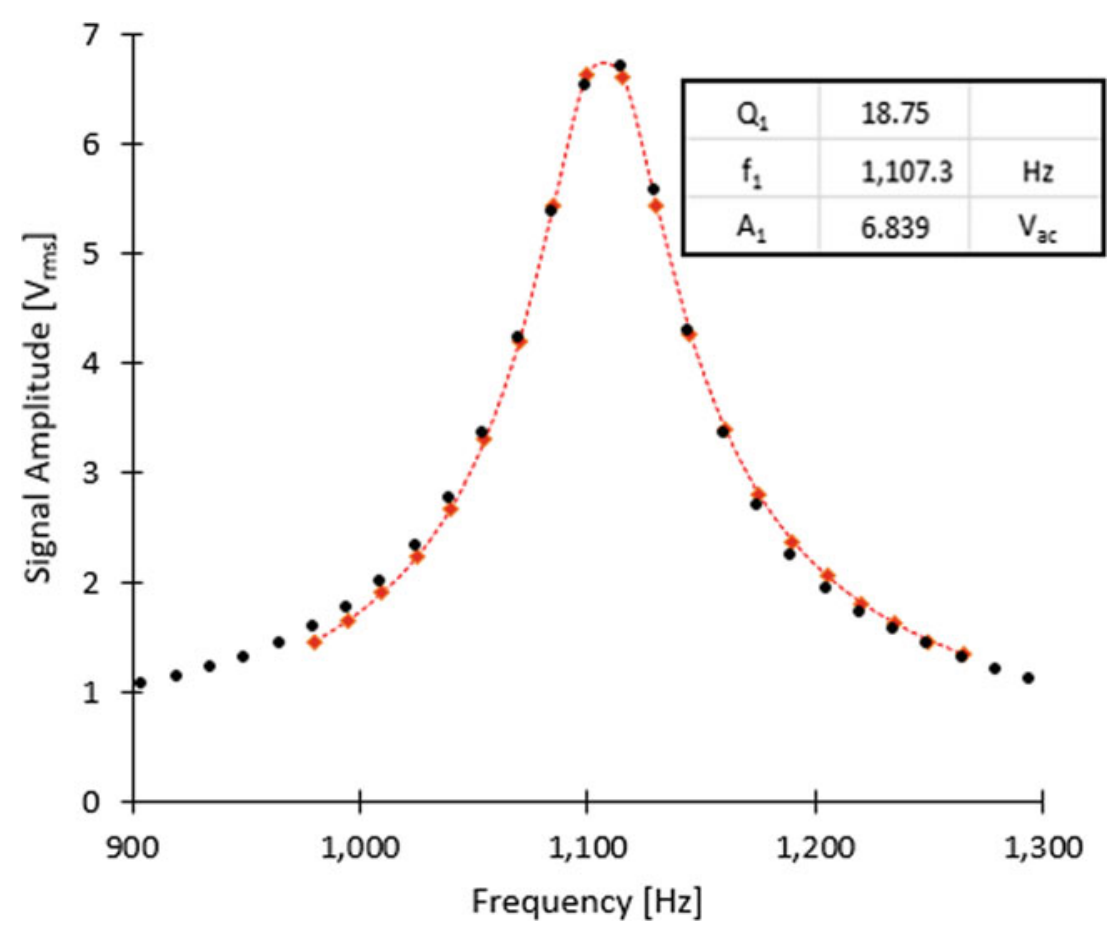

Fig. 2.13 Response of the $n=1$ torsional resonance of a bar similar to the fundamental mode shown in Fig. 5.16. The measured amplitudes, $A_{\text {exp }}(f)$, are shown as the 27 black dots plotted for $900 \leq f \leq 1300 \mathrm{~Hz}$. The red diamonds are values of $A_{1}\left(f / f_{1}\right)$ from Eq. (2.63) that have been joined by a smooth dotted line to illustrate the Rayleigh line shape. The parameter values in the box are those adjusted by a nonlinear curve-fitting algorithm (i.e., "solver") that minimized sum of the squared difference between $A_{\exp }(f)$ and $A_{1}\left(f / f_{1}\right)$

shape (aka Lorentz line shape) will describe systems with multiple resonances, like the modes of a long thin cylindrical bar in Fig. 5.16 or the modes of a gas-filled plane wave resonator driven by a loudspeaker in Figs. 10.12 and 10.14, as long as the individual modal frequencies are well-separated.

We can rewrite Eq. (2.62) in a more general form where the subscript, $n$, refers to the mode number. For the single-degree-of-freedom simple harmonic oscillator that has just been analyzed (or for the Helmholtz resonator discussed in Sect. 8.5), $n=0$. For the torsional resonance in Fig. 5.16, there are five resonances that would be designated $1 \leq n \leq 5$. At resonance, the amplitude of the response has its maximum value, $A_{n}(1)$.

$$
A_{n}\left(f / f_{n}\right)=\frac{A_{n}(1) / Q_{n}}{\sqrt{\left(\frac{f}{f_{n}}-\frac{f_{n}}{f}\right)^{2}+\frac{1}{Q_{n}^{2}}}}
$$

Here $Q_{n}$ is the quality factor of the $n$th mode, and $f_{n}$ is the resonance frequency of that $n$th mode. With $A_{n}(1)$, the frequency response function (i.e., line shape) created by Eq. (2.63) is characterized by three parameters: $A_{n}(1), f_{n}$, and $Q_{n}$.

We have already marveled at the ability of modern digital instrumentation and mathematical software to make it possible to acquire an array of data and fit those measurements to a chosen functional form. In Fig. 1.18, 2000 data points were acquired, and a nonlinear curve-fitting algorithm (i.e., "solver") was used to extract the five parameters necessary to characterize the free-decay of a 
loudspeaker that were specified in Eq. (1.119) by minimizing the sum of the squared difference between those measurements and the fitted function.

In Fig. 2.13, we executed a similar procedure to select the values of the three parameters in Eq. (2.63) that minimize the sum of the squared difference between the measured amplitude values, $A_{\text {exp }}(f)$ and $A_{n}\left(f / f_{n}\right)$, as was done for the least-squares fit to a straight line in Sect. 1.9. In this example, the response amplitudes were generated by the measurement of the fundamental $(n=1)$ resonant torsional modes of a spectrum like that in Fig. 5.15 but for a different bar. The best-fit values are shown in the boxed inset within Fig. 2.13. The slight asymmetry between the fit and the data is due to electromagnetic cross talk (see Sect. 5.4.3) that could be included within the model to improve the fit.

\subsubsection{Power Dissipation, the Decibel, and Resonance Bandwidth}

The time-averaged power dissipated in the mechanical resistance can be calculated using Eq. (1.73), and the mechanical impedance can be used to provide $v(t)$.

$$
\langle\Pi\rangle_{t}=\frac{1}{2} \mathfrak{R e}\left[\mathbf{F}^{*} \widehat{\mathbf{v}}\right]=\frac{F_{1}^{2}}{2} \mathfrak{R e}\left[\mathbf{Z}_{\mathbf{m}}^{-1}\right]=\frac{F_{1}^{2} R_{m}}{2\left|\mathbf{Z}_{\mathbf{m}}^{2}\right|}=\frac{1}{2} \frac{F_{1}^{2} R_{m}}{X_{m}^{2}+R_{m}^{2}}
$$

At resonance, $X_{m}\left(\omega_{o}\right)=0$, and the power dissipated in the mechanical resistance has its maximum time-averaged value, $\left\langle\Pi_{\max }\right\rangle_{t}$. The equation is analogous to the power dissipated in an electrical resistor with a potential difference amplitude, $V_{1}$, across an electrical resistance, $R_{e l}$.

$$
\left\langle\Pi\left(\omega_{o}\right)\right\rangle_{t}=\left\langle\Pi_{\max }\right\rangle_{t}=\frac{1}{2} \frac{F_{1}^{2}}{R_{m}}
$$

If instead we had used $\boldsymbol{Z}_{\boldsymbol{m}}$ to eliminate $\mathbf{F}=\mathbf{Z}_{\mathbf{m}} \widehat{\mathbf{v}}$ in Eq. (2.64), then we would have produced the analog to the electrical power dissipation which is proportional to the square of the current through the resistor.

$$
\left\langle\Pi\left(\omega_{o}\right)\right\rangle_{t}=\frac{1}{2} \mathfrak{R e}\left[\mathbf{F}^{*} \widehat{\mathbf{v}}\right]=\frac{v_{1}^{2}}{2} \mathfrak{R e}\left[\mathbf{Z}_{\mathbf{m}}\right]=\frac{1}{2} R_{m} v_{1}^{2}
$$

It is easy to see from Eq. (2.64) that the power is reduced to half of the maximum value when $R_{m}= \pm X_{m}$. There are two frequencies at which the power is half the maximum: $\omega_{+}$and $\omega_{-}$. Those two frequencies can be determined by solution of Eq. (2.58).

$$
m \omega_{+}-\left(\mathrm{K} / \omega_{+}\right)=R_{m} \quad \text { and } \quad m \omega_{-}-\left(\mathrm{K} / \omega_{-}\right)=-R_{m}
$$

Substituting $\mathrm{K}$ from the expression for $\omega_{-}$into the expression for $\omega_{+}$produces an equation that can be factored to determine the required frequency difference.

$$
\omega_{+}^{2}-\omega_{-}^{2}=\left(\omega_{+}-\omega_{-}\right)\left(\omega_{+}+\omega_{-}\right)=\frac{R_{m}}{m}\left(\omega_{+}+\omega_{-}\right)
$$

The expression for the quality factor from Eq. (2.44) generates another useful relation for $Q$ that emphasizes the fact that $Q$ is a dimensionless measure of the sharpness of a resonance.

$$
Q=\frac{\omega_{o} m}{R_{m}}=\frac{\omega_{o}}{\omega_{+}-\omega_{-}}=\frac{f_{o}}{f_{+}-f_{-}}=\frac{\sqrt{f_{+} f_{-}}}{f_{+}-f_{-}}=\frac{f_{o}}{(\Delta f)_{-3 \mathrm{~dB}}}
$$


Due to the symmetry of Eq. (2.62) with respect to $\omega / \omega_{\mathrm{o}}$ and $\omega_{\mathrm{o}} / \omega, f_{o}$ is the geometric mean of $f_{+}$and $f_{-}$, not their arithmetic average.

The right-hand expression introduces the "down $3 \mathrm{~dB}$ " bandwidth of the resonance, $(\Delta f)_{-3 \mathrm{~dB}}=f_{+}-f_{-}$. This is the full bandwidth of the resonance between the two half-power frequencies and is also known as the full width at half maximum, $(\Delta f)_{F W H M}$. The use of the decibel for expression of power ratios will be discussed further in Part II - Fluids, particularly for the specification of sound levels in air in Sect. 10.5. Briefly, the decibel (abbreviated "dB") was introduced in the early days of telephony as a means for using addition and subtraction to multiply transducer sensitivities, amplifier gains, and transmission losses in the calculation of the overall response of telephone systems in the century before digital computers became available to engineers and scientists.

$$
\mathrm{dB} \equiv 10 \log _{10}\left(\frac{\Pi_{1}}{\Pi_{2}}\right)
$$

In our case, $\Pi_{2}=\left\langle\Pi_{\max }\right\rangle_{t}$, the time-average power at resonance, and $\Pi_{1}=(1 / 2)\left\langle\Pi_{\max }\right\rangle_{t}$ is the half-power point: $10 \log _{10}(1 / 2)=-3.01$, hence, the "down $3 \mathrm{~dB}$ " designation.

Figure 2.14 plots the velocity amplitude response (above) and phase response (lower right) vs. frequency ratio, $f / f_{o}$, for a driven harmonic oscillator with three different values of quality factor, $Q$. Those amplitude response curves never cross. Driven with the same force, the system with the higher-quality factor will have larger displacement, velocity, and acceleration responses at all drive frequencies. The peak in the velocity response always occurs at $f_{o}$. If we were to plot the displacement response, the peak would occur slightly below $f_{o}$ since $\widehat{\mathbf{x}}=\widehat{\mathbf{v}} /(j \omega)$. Similarly, the peak in the acceleration response, $\widehat{\mathbf{a}}=(j \omega) \widehat{\mathbf{v}}$, would occur at a slightly higher frequency than $f_{o}$.

The relative power delivered to the mechanical resistance vs. the frequency ratio, $f / f_{o}$, is shown at the lower left of Fig. 2.14. The dashed lines, which intersect $(1 / 2)\left\langle\Pi_{\max }\right\rangle_{t}$, occur at $f_{-}$and $f_{+}$.

At this point, it is useful to derive still another expression for the quality factor, this time in terms of the energy stored in the spring and the mass at resonance, $E_{\text {Stored }}$, and the energy dissipated in the mechanical resistance during one cycle, $\left\langle\Pi_{\max }\right\rangle_{t} T$.

$$
\frac{E_{\text {Stored }}}{\left\langle\Pi_{\max }\right\rangle_{t} T}=\frac{\omega_{o} E_{\text {Stored }}}{2 \pi\left\langle\Pi_{\max }\right\rangle_{t}}=\frac{\frac{m}{2} v_{1}^{2} \omega_{o}}{2 \pi \frac{R_{m} v_{1}^{2}}{2}}=\frac{Q}{2 \pi}
$$

This form can be convenient for calculation of $Q$ since $E_{\text {Stored }}$ and $\left\langle\Pi_{\max }\right\rangle_{t}$ are both scalars.

Now that we have an expression for the power dissipated in the mechanical resistance of a damped driven simple harmonic oscillator, it is possible to calculate the equivalent noise bandwidth, $(\Delta f)_{E Q N B}$, as shown in Eq. (2.73), that was required for our reconciliation of the consequences of the Equipartition Theorem in Eqs. (2.49) and (2.50), and the fluctuating force in Eq. (2.51), by working backward to create an expression for the fluctuating force from the results of the Equipartition Theorem.

$$
\frac{1}{2} k_{B} T=\left\langle\frac{1}{2} m v^{2}\right\rangle=\frac{m}{2}\left\langle\frac{|F|^{2}}{\left|\mathbf{Z}_{\mathbf{m}}\right|^{2}}\right\rangle=\frac{m F_{\text {noise }}^{2}}{2} \int_{0}^{\infty} \frac{d f}{\left|\mathbf{Z}_{\mathbf{m}}\right|^{2}}
$$

Using the form of mechanical impedance from Eq. (2.60) and defining a dimensionless frequency ratio, $\Omega=f / f_{o}$, so $\mathrm{d} f=f_{o} \mathrm{~d} \Omega$, that definite integral can be evaluated using integral tables [20] or numerically [14]. 

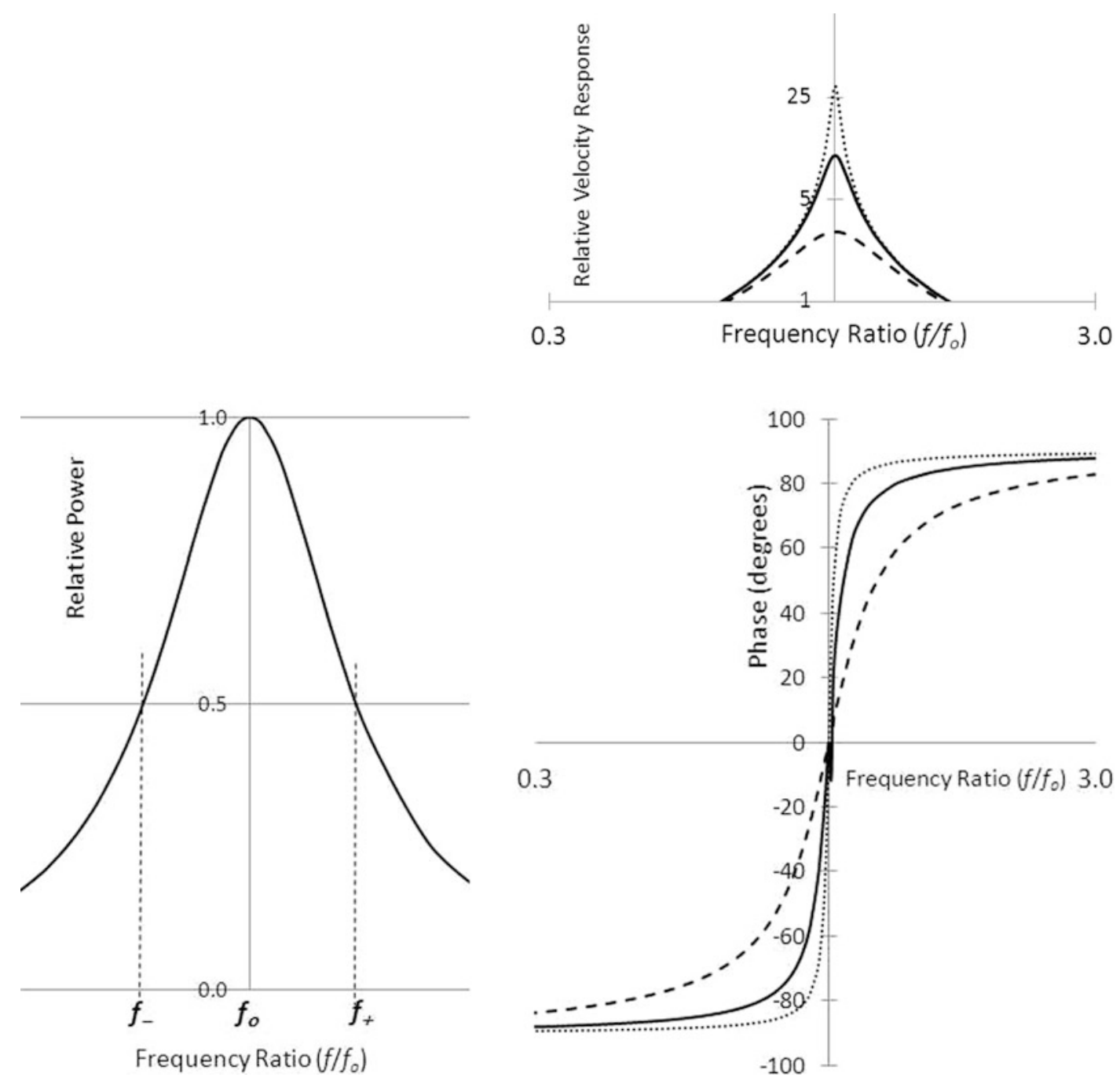

Fig 2.14 (Top right) Velocity amplitude response of a driven damped harmonic oscillator with $Q=3$ (dashed line), $Q=10$ (solid line), and $Q=30$ (dotted line) vs. the ratio of the drive frequency to the resonance frequency, $f l f_{o}$. Note that none of these lines cross. (Bottom right) Phase response vs. frequency ratio for the same three values of $Q$. (Bottom left) Relative power for $Q=3$ showing the resonance frequency, $f_{o}$; the lower $-3 \mathrm{~dB}$ frequency, $f_{-}$; and the upper $-3 \mathrm{~dB}$ frequency, $f_{+}: Q=f_{o} /\left(f_{+}-f_{-}\right)$

$$
\int_{0}^{\infty} \frac{d \Omega}{\frac{1}{Q^{2}}+\left(\Omega-\frac{1}{\Omega}\right)^{2}}=\frac{\pi Q}{2}
$$

Substitution of the integral back into Eq. (2.72) results in an expression for the average kinetic energy that is independent of $R_{m}$.

$$
\left\langle\frac{1}{2} m v_{1}^{2}\right\rangle=\frac{m}{2} \frac{4 k_{B} T R_{m}}{\omega_{o}^{2} m^{2}} f_{o} \frac{\pi Q}{2}=\frac{1}{2} k_{B} T
$$

The equivalent noise bandwidth, $(\Delta f)_{E Q N B}$, of the resonance can also be related to the integral in Eq. (2.73): $(\Delta f)_{E Q N B}=(\pi / 2)(\Delta f)_{-3 \mathrm{~dB}}$. Once again, as we saw in Sect. 2.3.3, we have a final result that 
is dependent upon a particular parameter (i.e., the noise introduced by the dissipative component, $R_{m}$ ) but which hides the explicit dependence on the parameter that governs that phenomenon.

\subsubsection{Resonance Tracking and the Phase-Locked Loop*}

Typically, we think of the peak in the amplitude response of a damped driven harmonic oscillator as being the primary characteristic of a resonator driven at its resonance frequency. There is certainly good reason for this prejudice, since resonance can be used to optimize the coupling of an oscillatory force to a useful load, for example, the coupling of an electrodynamic loudspeaker to a thermoacoustic resonator [21]. But we are well aware (see Sect. 1.2) that the slope of the amplitude vs. frequency at that response maximum (or any extremum) is zero. For that reason, if the drive is de-tuned from resonance (e.g., by a change in temperature or some other environmental effect that alters $\omega_{o}$ ), the amplitude of the response will decrease whether the de-tuning is either "flat" (i.e., drive frequency below resonance) or "sharp" (i.e., drive frequency above resonance). For that reason, a control system that monitors amplitude cannot determine whether to increase or to decrease the drive frequency to maintain the system on resonance based only on this change in amplitude.

A casual inspection of the resonance response curves in Fig. 2.14 shows that the rate of change of the phase difference with frequency, between the driving force and the velocity response, $\mathrm{d} \Theta / \mathrm{d} \omega$, is maximum at resonance, where $\Theta=0$. Because $\Theta \cong 0$ near the resonance frequency, $\omega_{o}$, it is easy to calculate that derivative, $(d \Theta / d \omega)_{\omega_{o}}$, using only the first term in the Taylor series for $\tan ^{-1} x \cong x .{ }^{15}$

$$
\left.\frac{d \Theta}{d \omega}\right|_{\omega_{o}}=\left.\frac{d\left(\tan ^{-1} X_{m} / R_{m}\right)}{d \omega}\right|_{\omega_{o}}=\left.\frac{1}{R_{m}} \frac{d}{d \omega}\left(\omega m-\frac{\mathrm{K}}{\omega}\right)\right|_{\omega_{o}}=\left[\frac{m+\frac{\mathrm{K}}{\omega^{2}}}{R_{m}}\right]_{\omega_{o}}
$$

Evaluation of that derivative at $\omega=\omega_{o}$, and substitution of $Q=\omega_{o} m / R_{m}$ and $\mathrm{K}=\omega_{o}^{2} m$, relates the rate of change of phase with respect to frequency to the quality factor.

$$
\left.\frac{d \Theta}{d \omega}\right|_{\omega_{o}}=\frac{2 m}{R_{m}}=\frac{2 Q}{\omega_{o}} \quad \text { or }\left.\quad \frac{d \phi}{d f}\right|_{f_{o}}=\frac{360^{\circ}}{\pi} \frac{Q}{f_{o}} \cong 114.6^{\circ} \frac{Q}{f_{o}}
$$

The right-hand version uses $\phi$ to indicate the phase difference in degrees, rather than $\Theta=\pi \phi / 180^{\circ}$, which was in radians, since the phase angle is more commonly reported in degrees by instrumentation or in computer models. This also produces another valuable expression for the quality factor in terms of the rate of change of phase difference with frequency at resonance.

$$
Q=\left.\left|\frac{\pi f_{o}}{360^{\circ}} \frac{d \phi}{d f}\right|_{f_{o}}|\cong| \frac{f_{o}}{114.6^{\circ}} \frac{d \phi}{d f}\right|_{f_{o}} \mid
$$

The absolute value is specified since the sign of the slope can be either positive or negative, depending upon the parity of the resonances, in systems with multiple resonances that occur for standing waves in continuous vibrators, like strings (see Sect. 3.3) or plane wave resonators (see Sect. 10.6).

In many circumstances, it is much easier to fit a straight line (see Sect. 1.9) to the phase difference vs. frequency, close to the zero-crossing (near resonance), to determine $Q$ than it is to use the $-3 \mathrm{~dB}$ bandwidth of Eq. (2.69). One such example is provided for the Helmholtz resonator in Sect.

\footnotetext{
${ }^{15}$ It is easy to see that $\tan ^{-1} x \cong x$ by inspection of the first term in the Taylor series expansion of $\tan x \cong x$ in Eq. (1.7) for small values of $x \ll 1$.
} 
8.5.2. The phase vs. frequency for that resonance is plotted near the resonance frequency in Figs. 8.32 and 8.33 .

Returning to the control question, we see that phase difference between the drive and the response generates an "error signal" proportional to the de-tuning that provides the information required by a control system to correct deviations between the drive frequency, $\omega$, and the natural frequency, $\omega_{o}$. It provides a parameter that has one sign if the system is driven sharp and the opposite sign if the system is driven flat. That error signal is nearly linear in its deviation for small frequency shifts away from the resonance frequency.

The extraction of such an error signal, proportional to the phase difference, can be accomplished by multiplication of the drive and response signals followed by a low-pass filtering of the product. ${ }^{16}$ The drive signal will be our "reference," having constant amplitude and a frequency, $\omega$. The reference waveform could include a phase shift, $\phi_{r}$, due to the response of electronics, cables, etc., but we will allow $\phi_{r}$ to have an adjustable component as well: $F(t)=F_{1} \sin \left(\omega t+\phi_{r}\right)$. The steady-state response (signal) will have an amplitude given by Eq. (2.62) and must also have the same frequency, $\omega$, since we assume a linear response, but it will have a different phase, $\phi_{s}$, that will combine $\Theta$ of Eq. (2.61) and any electronic phase shifts: $v(t)=v_{1} \cos \left(\omega t-\phi_{s}\right)$. The following trigonometric identity will simplify the resulting product.

$$
\begin{gathered}
(\sin x)(\cos y)=\frac{1}{2}[\sin (x+y)+\sin (x-y)] \\
F(t) v(t)=\frac{F_{1} v_{1}}{2}\left\{\sin \left[2 \omega t+\left(\phi_{r}+\phi_{s}\right)\right]+\sin \left(\phi_{r}-\phi_{s}\right)\right\}
\end{gathered}
$$

Both signals are assumed to be electrical waveforms generated by the diving force and some velocity transducer, and their product is also an electrical waveform. ${ }^{17}$ If the product is passed through a low-pass filter with a cut-off frequency, $2 \pi f_{-3 \mathrm{~dB}} \ll \omega$, then the component at twice the drive frequency will be strongly attenuated, and only a signal proportional to the sine of the phase difference will remain. If the electronically induced phase shifts are adjusted to make the phase difference $\left(\phi_{r}-\phi_{s}\right) \cong 0$ at resonance, then the output of the low-pass filter will be just the error signal that was sought for control of the drive frequency. ${ }^{18}$

The low-pass filtered signal can be fed back to a voltage-controlled oscillator ${ }^{19}$ to drive the system at its resonance frequency. This strategy, known as a phase-locked loop, is used in many applications outside of acoustics [22].

\footnotetext{
${ }^{16}$ The "multiplication" can be done either digitally or using analog electronics. Various analog schemes can be implemented ranging from a simple "optional inverter" [11] that synchronizes a square waveform with the reference to multiply the signal by \pm 1 to the use of a four-quadrant analog multiplication integrated circuit (e.g., Analog Devices AD633).

${ }^{17}$ Of course, it is possible that those signals were digitized prior to multiplication, and the operation of Eq. (2.79) was executed in a computer. For now, I will continue to treat the signals as analog electrical waveforms ('cause I'm an "old guy").

${ }^{18} \mathrm{An}$ integrator is often used instead of a low-pass filter since it can provide a non-zero output to the voltage-controlled oscillator that forces the phase difference to zero. If a low-pass filter is used, then a non-zero phase difference is required to provide the correction to the voltage-controlled oscillator, so the system will have to be driven slightly off-resonance to provide an adequate error signal. As the gain is increased to minimize the offset, the electronic system can become oscillatory (i.e., unstable).

${ }^{19}$ Analog integrated circuits that are voltage-controlled oscillators are readily available and very inexpensive for a wide variety of frequency ranges. For audio applications, I have used an Exar XR2206 that provides both a square-wave and low-distortion sine wave output and also includes a voltage-controlled amplifier to facilitate automatic gain control.
} 
Fig. 2.15 This block diagram illustrates the signal flow in a phaselocked loop frequency tracking circuit meant to maintain a driven harmonic oscillator at its resonance frequency by generation of an error signal proportional to the phase difference between the drive and the response. In this diagram, an integrator is used as the low-pass filter, since it can provide a non-zero output to control the VCO when the phase difference has been driven to zero. The mixer executes the multiplication in Eq. (2.79)

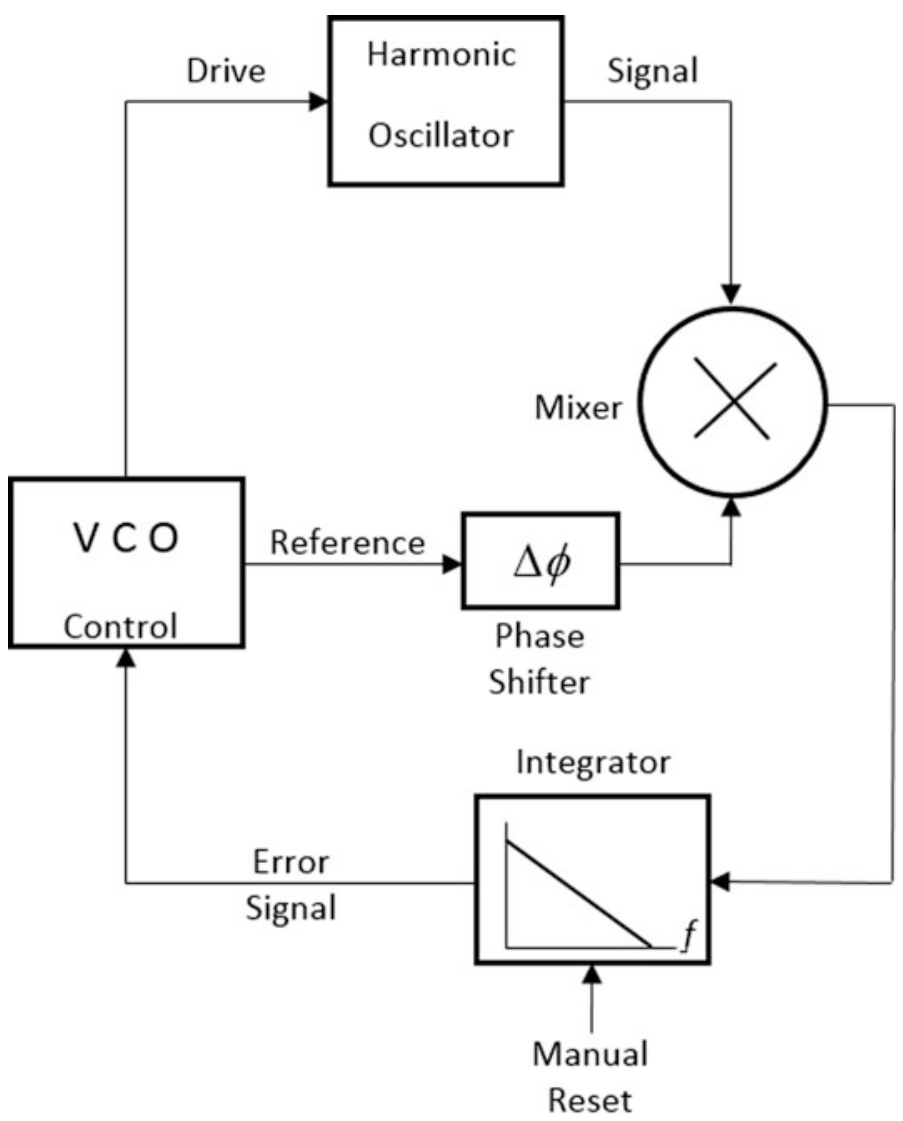

It is not the intention of this textbook to provide detailed electronic strategies for tracking resonances, but it is very important to appreciate the utility of the phase difference between the drive and response of a simple harmonic oscillator, as well as the frequency dependence of the amplitude. Such feedback control systems have to be designed carefully to avoid instabilities, but the combination of digital or analog electronics that can provide phase detection and stability at a level of $\pm 0.01^{\circ}$ at very modest cost, combined with the high rate of change of phase with frequency shown in Eq. (2.76) for resonators with even modest quality factors, is a very powerful tool in a variety of acoustical control applications. A block diagram of one possible implementation of such a frequency tracking phase-locked loop (PLL) is shown in Fig. 2.15.

\subsubsection{Transient Response}

Thus far, in our analysis of the damped driven simple harmonic oscillator, we have focused on the steady-state solution that is determined entirely by the oscillator's mechanical impedance, given in Eq. (2.58) or Eqs. (2.60) and (2.61). As mentioned earlier, the equation that describes the dynamics of the undriven system is identical to Eq. (2.55) but homogeneous. The solution to Eq. (2.39) can be added to the steady-state solution to incorporate the initial conditions at the time the harmonic forcing 
function, $F(t)=\mathfrak{R e}\left[\widehat{\mathbf{F}} e^{j \omega t}\right]$, is applied to the mass through the specification of the undetermined amplitude, $|\widehat{\mathbf{C}}|$, and phase, $\phi$, or at the time the excitation is removed.

$$
x(t)=|\widehat{\mathbf{C}}| e^{-t / \tau} \cos \left(\omega_{d} t+\phi\right)+\frac{|\widehat{\mathbf{F}}|}{\omega\left|\mathbf{Z}_{\mathbf{m}}\right|} \sin (\omega t-\Theta)
$$

The contribution of the transient term can initially interfere with the steady-state solution, but eventually the transient solution will diminish after a number of periods that spans any interval longer than $\tau$.

Possibly more important are the consequences of the transient term which follow the removal of the forcing function. The reason is that it is possible that the amplitude of the steady-state solution can become quite large, especially if the system is being driven at a frequency that is close to its natural (resonance) frequency, $\omega_{o}$. When the forcing ceases, the system will ring down, decaying at its damped frequency, $\omega_{d}$. The acoustic radiation from this ring down is a serious problem for sound reproduction systems at low frequencies producing undesirable "boominess in the bass."

An accomplished musician, playing an electric bass guitar, will frequently damp the motion of a string, abruptly cutting off the force that the instrument (though the pick-up and amplifier) applies to the loudspeaker. If the loudspeaker (in its enclosure) is significantly under-damped, the sound radiated after the electric bass's string has stopped vibrating will be dominated by the decay of the loudspeaker's motion. That sound will decay at $\omega_{d}$, independent of the note played by the bassist. In certain high-power automotive sound systems, this effect, caused by the ring down, can be particularly unpleasant.

The effects of the transient term in Eq. (2.80) provide insights into the growth and decay of the response of a damped, driven harmonic oscillator that are particularly useful when that system is driven at the resonance (natural) frequency, $\omega_{o}$. At $t=0$, the response is zero, so $C$ equals the steady-state amplitude and the transient term is $180^{\circ}$ out-of-phase with the steady-state solution. As the transient portion decays with an exponential time constant, $\tau$, the response amplitude grows. For early times, $t<\tau$, after the application of the force, the sum of the transient and the steady-state solution creates a maximum amplitude envelope, $|A(t)|$, that initially grows linearly with time.

$$
|A(t)|=A_{1}\left(1-e^{-t / \tau}\right)
$$

In this expression, $A_{1}$ is the steady-state amplitude response to the driving force. For times that are less that $\tau$, the exponential can be approximated by the first two terms in the Taylor series expansion of Eq. (1.4), so the initial growth of the amplitude envelope will be approximately a linear function of time after the application of the driving force, as shown in Fig. 2.16.

$$
|A(t)|=A_{1}\left(1-e^{-t / \tau}\right) \cong A_{1}\left[1-\left(1-\frac{t}{\tau}+\ldots\right)\right] \cong A_{1} \frac{t}{\tau} \text { for } t \leq \tau
$$

Using this linear approximation for the initial growth of the resonance, we can approximate the time, $t_{1 / 2}$, it takes for the amplitude of the resonance to grow to one-half of its steady-state value, since initially $\mathrm{d} A / \mathrm{d} t \cong(A / \tau)$.

$$
\frac{\left|A\left(t_{1 / 2}\right)\right|}{A_{1}}=\frac{1}{2} \cong \frac{t_{1 / 2}}{\tau}=\frac{Q}{2 \pi f_{o}}=\frac{Q}{2 \pi} T
$$

It takes approximately $(Q / 2 \pi)$ cycles for the response of a system driven at its resonance frequency to reach half its steady-state amplitude. 

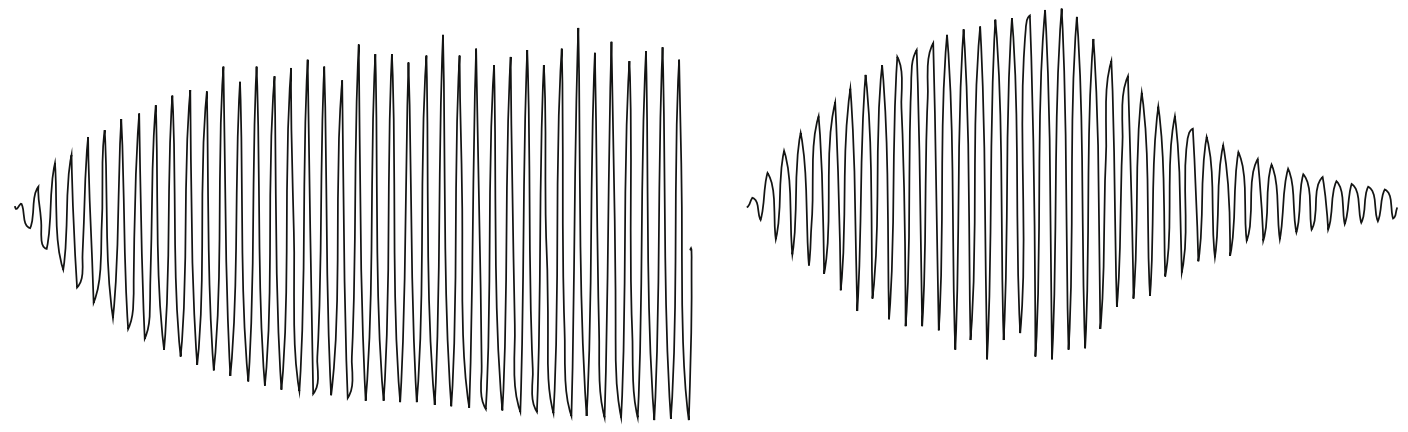

Fig. 2.16 The growth and decay of a damped harmonic oscillator driven at its natural frequency $\omega_{o}$. In this example, $Q \cong 25$. The response grows to about half of its steady-state value after about five cycles. Once the drive is cut off, the freely decaying sinusoid has a frequency, $\omega_{d}$, which has a relative difference from $\omega_{o}$ of only $0.02 \%$. (Figures courtesy of J. D. Maynard [23])

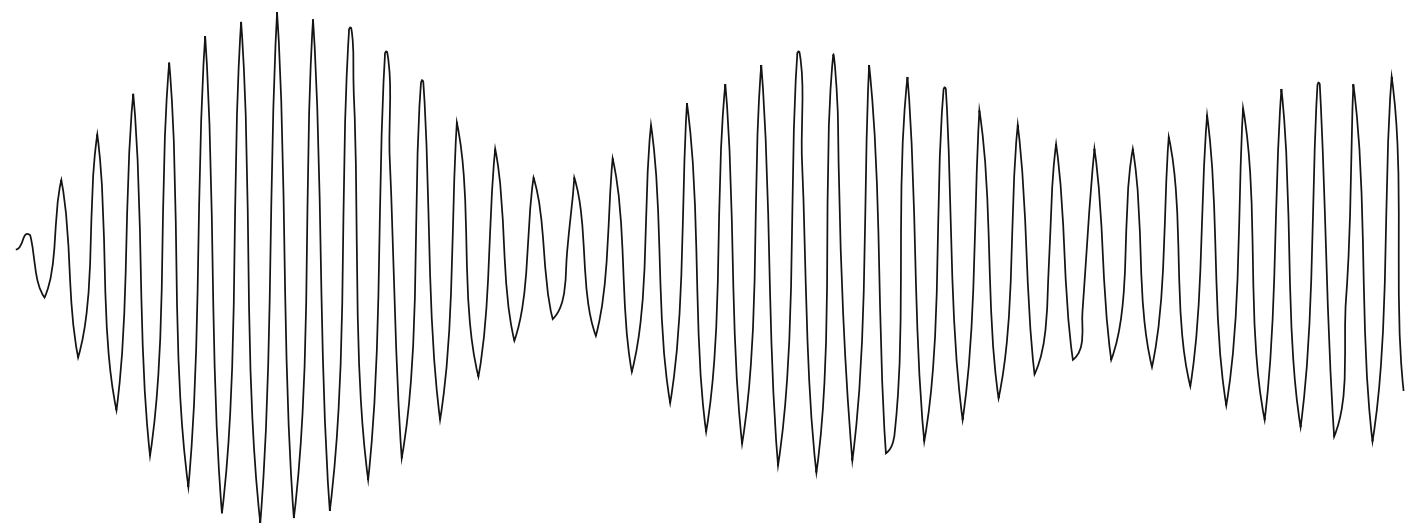

Fig. 2.17 This response is generated by an oscillator with $Q=80$ driven slightly off resonance. The transient solution beats against the steady-state solution. Forty cycles are shown, but after about 80 cycles, the transient contribution would be negligible. (Figures courtesy of J. D. Maynard [23])

Since the drive frequency is $\omega_{o}$ and the frequency of the transient contribution is $\omega_{d} \neq \omega_{o}$, one might ask whether those two solutions might not maintain the phase relation we assumed to fit the initial condition of zero amplitude as time increases. As calculated in Eq. (2.45), the relative differences in those two frequencies are small and are related to the square of the quality factor.

$$
\frac{\omega_{o}-\omega_{d}}{\omega_{o}} \cong \frac{1}{8 Q^{2}}
$$

By the time the resonance has grown to one-half its steady-state amplitude, the phase difference, $\varphi\left(t_{1 / 2}\right)$, between the transient and steady-state solutions, will equal $\varphi\left(t_{1 / 2}\right)=\delta \omega \bullet t_{1 / 2}=(8 Q)^{-1}=45^{\circ} / Q$, so de-phasing of those two terms of slightly different frequencies is insignificant for high- $Q$ systems.

If the driving frequency is slightly different from the natural frequency, the transient and the steadystate solutions can "beat" against each other until the transient solution has decayed. This behavior is illustrated in Fig. 2.17. When slightly off resonance, the transient and the steady-state solutions can destructively interfere to nearly zero; if the drive is turned off at that time, the ring-down is nearly eliminated. 


\subsubsection{The Electrodynamic Loudspeaker}

The moving-coil electrodynamic loudspeaker is a nearly perfect example of a force-driven, damped, simple harmonic oscillator. It is particularly useful because passing an alternating electrical current through the loudspeaker's voice coil provides an easy method to control the magnitude and frequency of the force applied to the moving mass, $m_{o}$ (i.e., cone, voice coil, and former). The parts of such a loudspeaker are shown schematically in Fig. 2.18 (right). The voice coil can also be used as a velocity transducer since it will produce an open-circuit voltage that is directly proportional to the velocity of the moving mass.

In this section, we will use data taken from a "typical" electrodynamic loudspeaker ${ }^{20}$ to determine the parameters that characterize the behavior of this simple harmonic oscillator: $m_{o}, \mathrm{~K}$, and $R_{m}$. The " $B \ell$ - product" is the transduction coefficient that connects the alternating electrical current, $I(t)=$ $\mathfrak{R e}\left[\widehat{\mathbf{I}} e^{j \omega t}\right]$, to the force on the voice coil: $F(t)=(B \ell) I(t)$. In this case, $(B \ell)$ is the product of the magnitude of the magnetic induction, $|\vec{B}|[\mathrm{T}],{ }^{21}$ times the length, $\ell[\mathrm{m}]$, of the wire wound around the "former" to create the voice coil. Of course, we want to determine all of these parameters as accurately as possible without causing any damage to the loudspeaker.

The accurate determination of a loudspeaker's parameters is crucial for the design of a speaker system or headphones that are intended to reproduce music and/or speech. It also can provide important insight into the consistency of the manufacturing processes. In this section, one measurement method is described that exploits the description that was developed in this chapter to understand the damped driven harmonic oscillator. Although this approach may not be appropriate for productionline testing, it can provide "ground truth" to certify the validity of a production-line testing procedure.
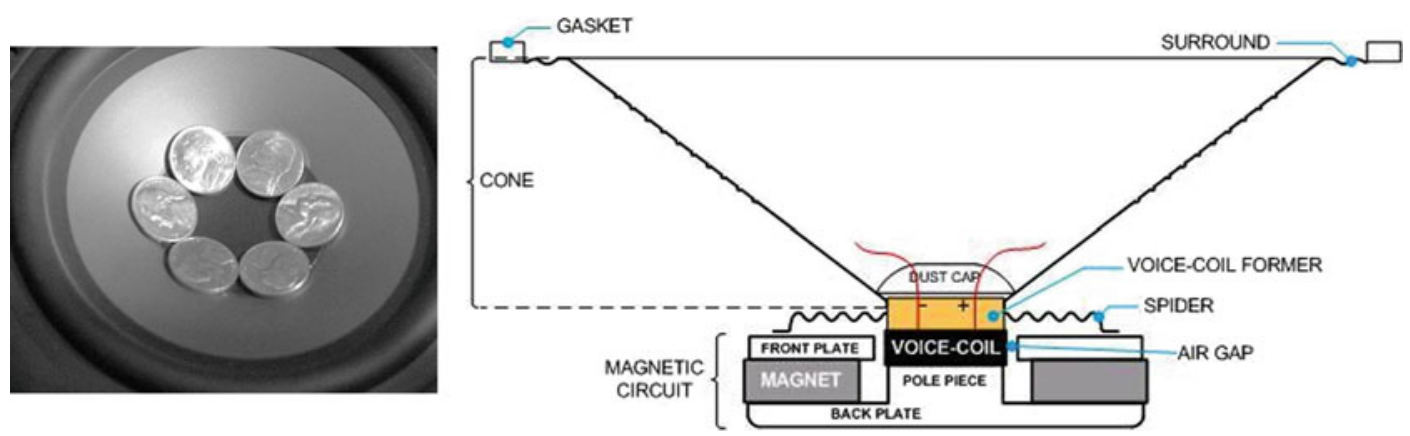

Fig. 2.18 (Left) Photograph looking down on a loudspeaker with six nickels resting on the cone surrounding the dust cap. (Right) Schematic representation of a moving-coil electrodynamic loudspeaker. The surround and spider center the voice coil in the air gap and provide the restoring stiffness, $\mathrm{K}$, to center the voice coil in the magnet gap. The mass of the voice coil, voice coil former, dust cap, and a portion of the masses of the surround and spider all contribute to the moving mass, $m_{o}$. The magnetic circuit, consisting of the permanent magnet material in a high magnetic permeability fixture (back plate, front plate, and pole piece), provides a radial magnetic flux, $|\vec{B}|$, in the air gap that is everywhere perpendicular to the wire of length, $\ell$, wound around the voice coil former

\footnotetext{
${ }^{20}$ Axon model 6S3 Mid-Bass Woofer with a cone diameter of about 6" $(16 \mathrm{~cm})$

${ }^{21}$ The tesla [T] is the SI unit of magnetic induction. The older c.g.s. unit is the gauss, which still appears in some of the loudspeaker literature: $1.0 \mathrm{~T}=10,000$ gauss.
} 
The stiffness, $\mathrm{K}$, and moving mass, $m_{o}$, can be determined by adding known masses, $m_{i}$, to the cone and measuring the loudspeaker's resonance frequencies, $f_{i}$, with each added mass. When the voice coil is driven by a constant current source (i.e., the current is independent of the electrical load presented by the voice coil), ${ }^{22}$ the voltage across the voice coil has its maximum value at resonance. This is a direct consequence of Lenz's Law, which is responsible for the minus sign in Faraday's law of induction: $V=-(d \Phi / d t)$. The magnetic flux, $\Phi$, is the product of the magnitude of the magnetic induction, $|\vec{B}|$, and an area with a normal that is perpendicular to the direction of $\vec{B}$. The "back electromotive force (emf)," represented by the induced voltage, $V$, will have its maximum value at resonance since the rate of change of the flux is proportional to the velocity of the voice coil.

Convenient masses to use for such an experiment are the US nickel, which weighs exactly 5.000 g. ${ }^{23}$ Care must be taken to keep the electrical current through the voice coil at a low enough level that the peak acceleration of the loudspeaker cone never exceeds the acceleration due to gravity. At greater accelerations, the coins will "bounce," since they cannot fall back fast enough to track the displacement of the cone. The data acquired for measurement of the resonance frequencies, $f_{i}$, corresponding to the addition of $m_{i}=(0.005 \mathrm{~kg}) i$, is tabulated on the left side of Fig. 2.19 for $i=0,1,2,3,4$, and 5 .

The natural frequency of the loudspeaker, with the added masses, is given simply by the square root of the ratio of the suspension stiffness, $\mathrm{K}$, and the total moving mass, $m_{o}+m_{i}$.

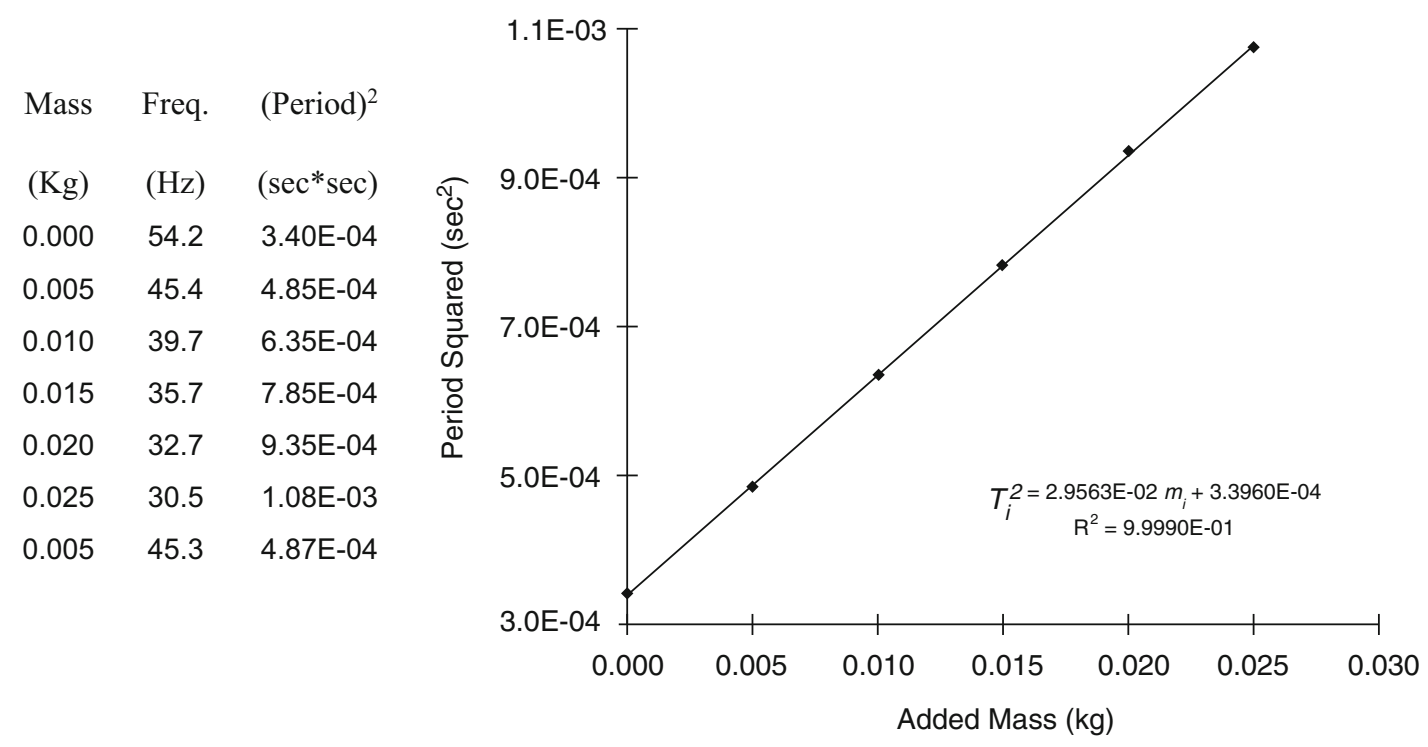

Fig. 2.19 (Left) Table of frequencies and squared periods for an electrodynamic loudspeaker's resonance with between zero and five US nickels placed on the cone (see Fig. 2.18). (Right) Plot of period squared, $T_{i}^{2}$, vs. added mass, $m_{i}$. $R^{2}$ is the square of the correlation coefficient (see Sect. 1.9.1)

\footnotetext{
${ }^{22}$ In practice, it is easy to approximate a constant current source by placing an electrical resistor, $R_{c}$ (commonly referred to as the "current limiting resistor"), in series with the output of a signal (voltage) generator that is large compared to the largest magnitude of the loudspeaker's input electrical impedance $\left|\boldsymbol{Z}_{\boldsymbol{e}}\right| \ll R_{c}$. For these measurements, $\left|\boldsymbol{Z}_{\boldsymbol{e} l}\right|<100 \Omega$, so $R_{c} \cong 1 \mathrm{k} \Omega$ was adequate to keep the current sufficiently constant.

${ }^{23}$ The US Mint website provides mass specifications for US coinage: www.usmint.gov. The US penny is 2.500 gm.
} 


$$
f_{i}=\frac{1}{2 \pi} \sqrt{\frac{\mathrm{K}}{m_{o}+m_{i}}}
$$

That equation has only two unknown parameters: $m_{o}$ and $\mathrm{K}$. The technique of linearized least-squares fitting, introduced in Sect. 1.9.3, can be used to transform Eq. (2.85) into a linear relationship between the square of the period, $T_{i}^{2}=\left(f_{i}^{2}\right)^{-1}$, and the added masses, $m_{i}$.

$$
\frac{1}{f_{i}^{2}}=T_{i}^{2}=\frac{4 \pi^{2}}{\mathrm{~K}}\left(m_{0}+m_{i}\right)=\frac{4 \pi^{2}}{\mathrm{~K}} m_{0}+\frac{4 \pi^{2}}{\mathrm{~K}} m_{i}
$$

In this form, the slope of the line plotted in Fig. 2.19 (Right) is $4 \pi^{2} / \mathrm{K}$. The value of the intercept divided by the value of the slope provides the moving mass, $m_{o}$.

Visual inspection of the graph in Fig. 2.19 suggests that the tabulated measurements are wellrepresented by Eq. (2.86). The techniques described in Sect. 1.9.2 relate the square of the correlation coefficient, $R^{2}$, to the relative uncertainty in the slope, providing the following value for the measured suspension stiffness and its uncertainty based on those six measured resonance frequencies: $\mathrm{K}=1335 \pm 7 \mathrm{~N} / \mathrm{m}( \pm 0.5 \%)$. Here, the reported uncertainty corresponds to one standard deviation $( \pm 1 \sigma)$.

The relative uncertainty in the intercept is $\pm 0.6 \%$. Since the moving mass involves the ratio of the intercept to the slope, the relative uncertainties in both the slope and the intercept need to be combined in a Pythagorean sum, as discussed in Sect. 1.8.4.

$$
\frac{\delta m_{o}}{\left|m_{o}\right|}=\left[\left(\frac{\delta(\text { slope })}{\mid \text { slope } \mid}\right)^{2}+\left(\frac{\delta(\text { intercept })}{\mid \text { intercept } \mid}\right)^{2}\right]^{1 / 2}=\sqrt{(0.5 \%)^{2}+(0.6 \%)^{2}}=0.8 \%
$$

This results in an experimental value for the loudspeaker's moving mass: $m_{o}=(11.5 \pm 0.1) \times 10^{-3}$ $\mathrm{kg}$. Again, the estimated error reflects an uncertainty of one standard deviation $( \pm 1 \sigma)$.

The mechanical resistance can be determined from a simple free-decay experiment. I find it convenient to build a simple switching circuit $^{24}$ that allows me to drive the speaker near its resonance frequency and then cut the drive while simultaneously triggering a digital oscilloscope and recording the voltage generated by the voice coil that is then connected to the input of that oscilloscope (see Fig. 1.18), which is directly proportional to the velocity of the voice coil.

The data from the free-decay measurement on this loudspeaker were used as the example for fitting an exponentially decaying signal to a straight line by taking the logarithm of the output voltage differences between successive peaks and troughs of the damped sinusoidal waveform. The natural log of these voltage differences is plotted in Fig. 1.15. The linearized least-squares fit produced an exponential decay time of $\tau=(22.18 \pm 0.17) \times 10^{-3} \mathrm{~s}$. A fit to the cycle number vs. the time for the voltage zero-crossings, using the same data, provided a measurement of the free-decay frequency and its uncertainty: $f_{d}=51.82 \pm 0.17 \mathrm{~Hz}$.

The mechanical resistance is related to both the decay time, $\tau$, and the moving mass, $m_{o}, R_{m}=2 m_{o}$ l $\tau$, so the relative uncertainties for both quantities have to be combined in a Pythagorean sum, as was done previously in Eq. (2.87). This results in a measured value of $R_{m}=1.04 \pm 0.01 \mathrm{~kg} / \mathrm{s}$.

\footnotetext{
${ }^{24}$ Such a switch can be as simple as a double-pole double-throw toggle switch with one pole controlling a battery that provides a triggering voltage to the oscilloscope and the other pole used to move the voice coil from the output of the signal generator to the input of the oscilloscope that acquires and stores the free-decay time history, like the one shown in Fig. 1.18 and analyzed in Fig. 1.15.
} 
Having determined $R_{m}$, it is possible to measure the $\mathrm{B} \ell$-product if the $\mathrm{DC}$ electrical resistance of the voice coil, $R_{d c}$, is known, and the magnitude of the electrical impedance at resonance, $\left|\mathbf{Z}_{\mathbf{e l}}\left(f_{o}\right)\right|=V_{a c}\left(f_{o}\right) / I_{a c}\left(f_{o}\right)$, is measured. At resonance, the voltage and current are in-phase (thus allowing use of the rms values of $V$ and $I$ ), if the voice coil's inductance, $L$, can be neglected: $\omega_{o} L \ll R_{d c}$. At resonance, $X_{m}=0$, and the expression for the velocity of the voice coil is very simple in the resistance-controlled regime.

$$
v\left(f_{o}\right)=\frac{F}{R_{m}}=\frac{(B \ell) I}{R_{m}}
$$

A voltage across the voice coil will be produced, in accordance with Faraday's law, by the back-emf, $V_{e m f}$, generated by the velocity, $v$, of the voice coil of length, $\ell$, moving in the magnetic field of magnetic induction, $|\vec{B}|$.

$$
V_{\text {emf }}\left(f_{o}\right)=(B \ell) v=\frac{(B \ell)^{2} I}{R_{m}}
$$

The ohmic voltage across the voice coil, $V_{\Omega}$, is due to the current through $R_{d c}: V_{\Omega}=R_{d c} I$. Since both voltages are in-phase with the current, the magnitude of the total electrical impedance measured at resonance, $\left|\mathbf{Z}_{\mathbf{e l}}\left(f_{o}\right)\right|$, is just the sum of those two voltages divided by the electrical current, $I$.

$$
\left|\mathbf{Z}_{\mathbf{e l}}\left(f_{o}\right)\right|=\left|\frac{V}{I}\right|_{f_{o}}=\frac{(B \ell)^{2}}{R_{m}}+R_{d c} \quad \Rightarrow \quad(B \ell)=\sqrt{R_{m}\left(\left|\mathbf{Z}_{\mathbf{e l}}\left(f_{o}\right)\right|-R_{d c}\right)}
$$

This method for measurement of the $B \ell$-product will not have the same accuracy as the mechanical measurements for $m_{o}, \mathrm{~K}$, and $R_{m}$, since $R_{m}$ was measured when the voice coil was an open circuit and there is a non-zero current passing through the voice coil when $\left|\mathbf{Z}_{\mathbf{e l}}\right|$ is being measured. This introduces other losses related to eddy currents induced in the metallic portions of the magnetic circuit and hysteresis in the orientation of the domains of the permanent magnetic materials [24], but with a reasonably large current-limiting resistor, these additional error sources can be minimized.

All of the moving-coil loudspeaker's mechanical parameters have been determined with a relative uncertainty of $\pm 1 \%$ or less using a few coins, an electrical resistor to hold current constant, a signal generator, an AC voltmeter, and a digital storage oscilloscope. This approach is so simple it can be used as a teaching laboratory exercise for college freshmen in a first-year seminar [25]. The fact that such simple, inexpensive, and highly accurate determinations of the parameters that characterize the linear behavior of electrodynamic loudspeakers are not widely appreciated, as evidenced by the following quotation [26]:

The added mass technique, discussed by Beranek [27] and others, requires a known, appreciable mass to be carefully attached to a diaphragm with appropriate distribution and driver orientation. The enclosed volume technique, ${ }^{25}$ discussed by Thiele [28] and other, requires the use of an airtight box with known enclosed volume after a driver has been mounted to it. Both approaches can be time consuming and problematic in implementation, with relative bias errors often reaching $10 \%$ or higher.

\footnotetext{
${ }^{25}$ The "enclosed volume technique" is similar to the added mass technique just described except instead of adding masses; the gas stiffness of the enclosure is used to measure the resonance frequency while changing the total stiffness. See Problem 4 at the end of this chapter.
} 


\subsubsection{Electrodynamic (Moving-Coil) Microphone}

The electrodynamic loudspeaker is a reversible transducer. Sound is generated by placing an alternating electrical current through the loudspeaker's voice coil. If the same speaker is placed into a sound field, the oscillating pressure difference (assuming the back of the speaker's cone is isolated from the oscillating pressure) of the sound will cause the speaker's diaphragm to vibrate.

If we start the analysis by assuming that the flow resistance shown in Fig. 2.20 (Left) is infinite, then the air inside the microphone remains at a constant pressure, $p_{m}$, and the excess pressure due to one frequency component of a sound wave that impinges on the microphone's diaphragm can be represented as $p(t)=\mathfrak{R e}\left[\widehat{\mathbf{p}} e^{j \omega t}\right]$. If the microphone has an area, $A_{\text {mic }}$, then the force on the diaphragm is $F_{1}(t)=A_{\text {mic }} \mathfrak{R e}\left[|\widehat{\mathbf{p}}| e^{j \omega t}\right]$. The microphone's moving mass (i.e., diaphragm and coil); suspension stiffness, $\mathrm{K}$; and mechanical resistance, $R_{m}$, determine the microphone's (complex) mechanical impedance, $\mathbf{Z}_{\mathbf{m}}$, so that the velocity of the coil is given by Eq. (2.62). Again, by Faraday's law, $V_{\text {emf }}(t)=(B \ell) v_{1}(t)$.

Operating as just described, such an electrodynamic microphone would have very uneven frequency response. During the second half of the twentieth century, small loudspeakers were used as both a sound source and a microphone in intercommunication systems for multistory dwellings. A resident could listen to someone at the front door and then press a switch that changed the mode of operation of the loudspeaker into a microphone and hear the voice of the visitor. In such an application, the fidelity was adequate for speech intelligibility.

The success of the electrodynamic microphone in both sound reinforcement and recording applications was due to Benjamin Baumzweiger (who later changed his name to Bauer), in 1937, who added the flow resistance shown in Fig. 2.20 (left) [29]. As just described, if the oscillating acoustic pressure is only applied to the outer surface of the microphone diaphragm, then the response of the microphone will be omnidirectional, if the wavelength of the sound is greater than the size of the diaphragm. If the acoustic pressure could reach both sides of the diaphragm, then the microphone would have a figure-eight (bi-directional) response pattern [30]. Bauer realized that a resistive element, in combination with the internal volume of the microphone, would provide access for the pressure at a small distance from the front of the microphone which could produce an intermediate (cardioid)
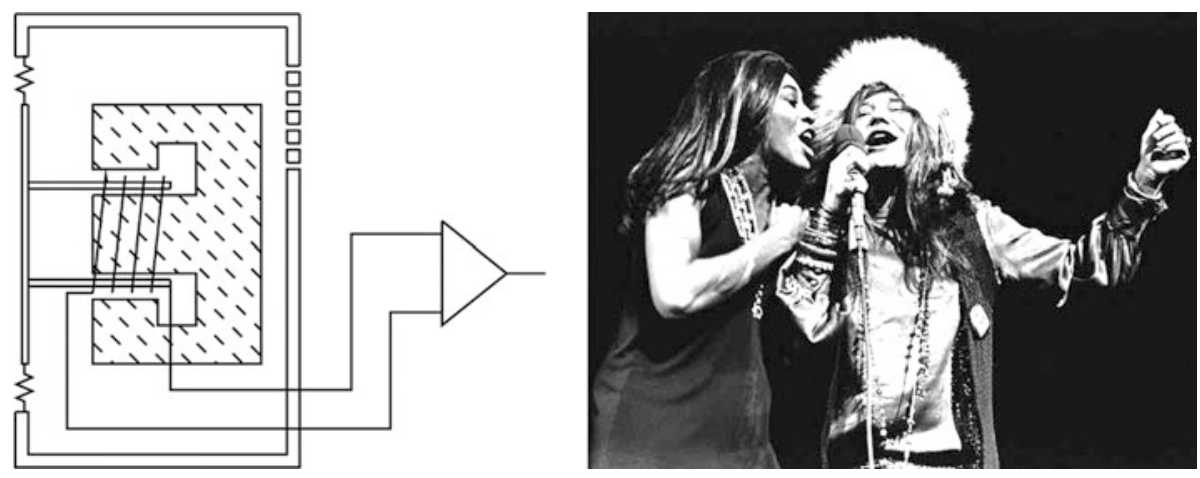

Fig. 2.20 (Left) Schematic diagram of an electrodynamic (moving-coil) microphone. An oscillatory pressure difference between the outside and the inside of the microphone's case exerts an oscillating force on the diaphragm causing the coil (shown connected to the input of an amplifier) to vibrate within the radial magnetic field produced by the hatched magnet structure. At the upper right of the microphone's enclosure is a porous medium that provides a flow resistance. Silk cloth is commonly used to provide the required flow resistance (see Sect. 12.5.3) (Diagram courtesy of J. D. Maynard). (Right) An electrodynamic microphone is shared by Tina Turner and Janis Joplin (Photo by Amalie R. Rothschild from the collection of Susan Levenstein and Alvin Curran) 
Fig. 2.21 The Shure Model 55 "Unidyne ${ }^{\circledR}$ ", (right) was the first product to incorporate the electrodynamic microphone strategy diagrammed schematically in Fig. 2.19 (left). (Left) The later model SM58 used a similar capsule. It is shown in use (with windscreen) in Fig. 2.19 (right). (Images used by permission, Shure, Inc.)

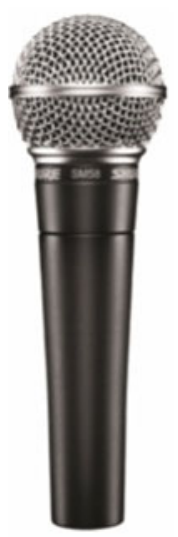

directivity pattern and that making the mechanical system resistance-controlled, the frequency response would be fairly uniform over a large range of frequencies. ${ }^{26}$

The first such single-element unidirectional electrodynamic microphone that used a single diaphragm, back-vented through a flow resistance, is the iconic Shure "Unidyne ${ }^{\circledR}$ " Model 55 shown in Fig. 2.21 (right) [31]. Later models, with the same type of microphone capsule, appeared in other packages like the Model SM58, shown in Fig. 2.21 (left), which has been the best-selling vocal microphone for the past 30 years.

\subsubsection{Displacement-Driven SHO and Transmissibility}

The decision to first analyze a damped simple harmonic oscillator, driven by a single-frequency sinusoidal force of constant amplitude, $\mathbf{F}$, that is applied to the mass, was an arbitrary choice. In this section, the response of the same system will be analyzed but driven by a constant amplitude displacement, $x_{o}(t)=\mathfrak{R e}\left[\widehat{\mathbf{x}} e^{j \omega t}\right]$, applied at the end of the spring that is not attached to the mass. Since the driving frequency, $\omega$, is determined, the effects of a constant displacement, constant velocity, or constant acceleration that is applied to the end of the spring will be similar, differing only by one or two factors of $j \omega$.

This situation is diagrammed schematically in Fig. 2.22. Such an arrangement is fairly typical of a vibration isolation system that is intended to support a sensitive instrument (phonograph, microphone, disk drive, atomic force microscope, etc.) and isolate it from the vibrations of the foundation on which it is supported.

The mass will be accelerated due to the force applied by the spring and by the dashpot.

$$
\mathrm{K}\left(x_{o}-x_{2}\right)-R_{m} \dot{x}_{2}=m \ddot{x}_{2} \quad \text { or } \quad \mathrm{K} x_{o}=m \ddot{x}_{2}+R_{m} \dot{x}_{2}+\mathrm{K} x_{2}
$$

Dividing this equation by $m$ and letting $x_{o}(t)=\mathfrak{R e}\left[\widehat{\mathbf{x}} e^{j \omega t}\right]$ transforms the above differential equation into an algebraic equation

\footnotetext{
${ }^{26}$ A systematic analysis of the directivity pattern of acoustic sensors will be provided in Sect. 12.5.
} 


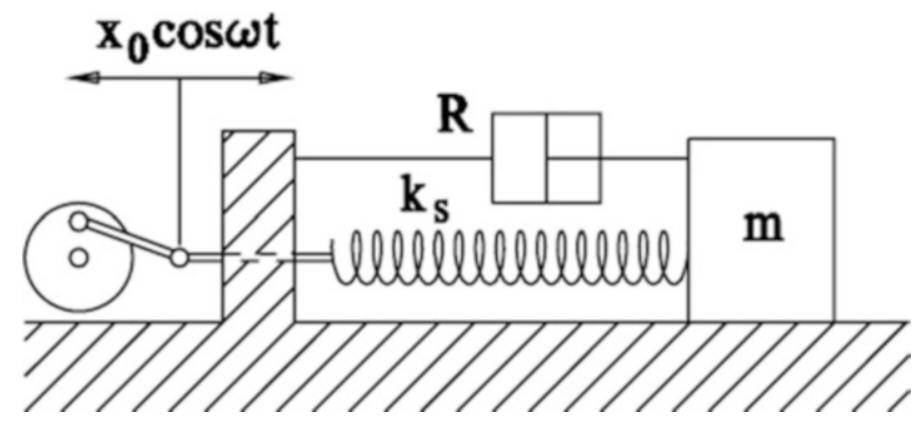

Fig. 2.22 In this configuration, a sinusoidal displacement is applied to the end of the spring located at $x_{o}$. A mass is attached to the other end of the spring with the junction between the mass and spring defining the position $x_{2}(t)$. One terminal of a dashpot, having a mechanical resistance, $R_{m}$, is attached to the mass, and the other terminal is rigidly fixed so the dashpot exerts a resistive force, $F=-R_{m}\left(d x_{2} / d t\right)$. (Diagram courtesy of J. D. Maynard)

$$
\omega_{o}^{2} x=-\omega^{2} x_{2}-j \omega \frac{R_{m}}{m} x_{2}+\omega_{o}^{2} x_{2}
$$

The steady-state solution for the mass's displacement, $x_{2}(t)$, is then rather reminiscent of our forcedriven solution in Eq. (2.57).

$$
x_{2}(t)=\mathfrak{R e}\left[\frac{\omega_{o}^{2} \widehat{\mathbf{x}} e^{j \omega t}}{\left(\omega_{o}^{2}-\omega^{2}\right)+j \omega \frac{R}{m}}\right]=\mathfrak{R e}\left[\frac{m \omega_{o}^{2} \widehat{\mathbf{x}} e^{j \omega t}}{j \omega\left[R_{m}+j\left(\omega m-\frac{k}{\omega}\right)\right]}\right]
$$

If the mass's response is expressed in terms of its complex velocity, $\mathbf{v}_{\mathbf{2}}(t)=\dot{\mathbf{x}}_{\mathbf{2}}(t)=j \omega \mathbf{x}_{\mathbf{2}}(t)$, then the steady-state solution that was expressed in terms of our complex mechanical impedance, $\mathbf{Z}_{\mathbf{m}}$, is recovered, as though $m \omega_{o}^{2} x_{o}$ had been substituted for $F_{1}$ in Eq. (2.57).

$$
\widehat{\mathbf{v}}_{\mathbf{2}}=\widehat{\hat{\mathbf{x}}}_{\mathbf{2}}=\frac{m \omega_{o}^{2} x_{o}}{\mathbf{Z}_{\mathbf{m}}}
$$

Many features of this solution should now be familiar. When driven at the resonance frequency, $\omega_{o}$, the displacement amplitude of the mass, $x_{2}$, is "amplified" by the quality factor of the oscillator.

$$
\left|\frac{\widehat{\mathbf{x}}_{\mathbf{2}}}{\widehat{\mathbf{x}}}\right|_{\omega_{o}}=\frac{\omega_{o}^{2} m}{\omega_{o} R_{m}}=\frac{\omega_{o} m}{R_{m}}=Q
$$

If the frequency of the drive, $\omega$, is much greater than $\omega_{o}$, the oscillator operates in its mass-controlled regime, so that the motion of the mass is reduced from that of the foundation.

$$
\lim _{\omega \gg \omega_{o}}\left|\frac{\widehat{\mathbf{x}}_{\mathbf{2}}}{\mathbf{x}_{\mathbf{o}}}\right|=\lim _{\omega \gg \omega_{o}}\left|\frac{\widehat{\mathbf{v}}_{\mathbf{2}}}{\dot{\mathbf{x}}_{\mathbf{o}}}\right|=\lim _{\omega \gg \omega_{o}}\left|\frac{\widehat{\mathbf{a}}_{\mathbf{2}}}{\ddot{\mathbf{x}}_{\mathbf{0}}}\right|=\frac{\omega_{o}^{2}}{\omega^{2}}
$$

In this mass-controlled limit, the displacement, velocity, or acceleration decreases by a factor of four for each reduction in $\omega_{o}$ by a factor of two. Of course, it is possible to stack multiple harmonic oscillator stages in series and produce even larger reductions in the transmissibility of a vibration isolator.

The similarity between the force-driven and displacement-driven cases means that the same strategy can be used to reduce the force generated by a vibrating piece of machinery. In the case of a vibration isolator, the foundation moves both the spring and the damper. This is not the same as Fig. 2.22, where 
one end of the resistance is fixed but is mechanically identical to the driven foundation of Fig. 2.23, where the mass is connected to the foundation through both the stiffness and the resistance elements.

As discussed in the following treatment of vibration sensors, our interest is in the relative motion of the mass and its foundation, $\mathbf{z} \equiv \mathbf{x}_{\mathbf{1}}-\mathbf{x}_{\mathbf{2}}$. Equation (2.91) can be rewritten by substituting $\mathbf{z}=\mathbf{x}_{\mathbf{1}}-\mathbf{x}_{\mathbf{2}}$.

$$
m \ddot{\mathbf{z}}+R_{m} \dot{\mathbf{z}}+\mathrm{Kz}=-m \ddot{\mathbf{x}}_{\mathbf{1}}=m \omega^{2} \widehat{\mathbf{x}}_{\mathbf{1}} e^{j \omega t}
$$

The complex transmissibility, $\boldsymbol{T}$, is defined as the ratio of the motion of the mass, relative to the foundation, $\mathbf{z}$, divided by the motion of the foundation, $\mathbf{x}_{\mathbf{1}}$, or the ratio of the force on the mass, $\mathbf{F}_{\mathbf{2}}$, to the force on the foundation, $\mathbf{F}_{\mathbf{1}}$ [32]. If the drive frequency, $\omega$, is written in terms of the natural frequency, $\omega_{o}$, so $\Omega=\omega / \omega_{o}$, and the mechanical resistance is written in terms of the quality factor, $R_{m}=\omega_{o} m / Q$, then the magnitude of the transmissibility, $|\boldsymbol{T}|$, and the phase angle between the displacements or forces, $\theta_{T}$, based on Eq. (2.93), can be put in a general form.

$$
\begin{gathered}
|\mathbf{T}|=\left|\frac{\mathbf{z}}{\mathbf{x}_{\mathbf{1}}}\right|=\left|\frac{\mathbf{F}_{\mathbf{2}}}{\mathbf{F}_{\mathbf{1}}}\right|=\frac{\sqrt{1+(\Omega / Q)^{2}}}{\sqrt{\left(1-\Omega^{2}\right)^{2}+(\Omega / Q)^{2}}} \\
\theta_{T}=\tan ^{-1}\left[\frac{-\Omega^{3} / Q}{1-\Omega^{2}+(\Omega / Q)^{2}}\right]
\end{gathered}
$$

A plot of $|\boldsymbol{T}|$ and $\theta_{T}$ will look identical to those for the driven harmonic oscillator in Fig. 2.14. Similarly, the frequency dependence of the transmissibility of a damped harmonic oscillator (DHO) is compared to the transmissibility for vibration isolators using rubber springs is presented in Sect. 4.5.3 in Figs. 4.30, 4.31, and 4.33.

\subsection{Vibration Sensors}

The displacement-driven one-dimensional simple harmonic oscillator provides the basis for most common vibration sensors. Typically, such a sensor is housed in a case that will be mounted on a vibrating surface. A schematic diagram of such a sensor is shown in Fig. 2.23. Since the transduction

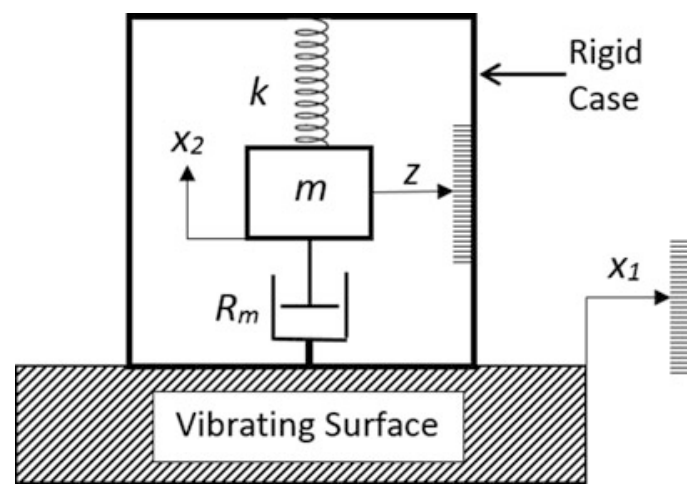

Fig. 2.23 Generic vibration sensor contained within a rigid case that is attached to a vibrating surface. The displacement of the vibrating surface is given by $\mathbf{x}_{\mathbf{1}}(t)$ and the motion of the mass, $m$, is given by $\mathbf{x}_{2}(t)$. The displacement of the mass, relative to the case, is given by $z$, where the scale inside the case represents the sensor's transduction mechanism. Scales are provided for $x_{1}$ and for $z$, where $x_{1}$ and $x_{2}$ are measured relative to a fixed coordinate system 

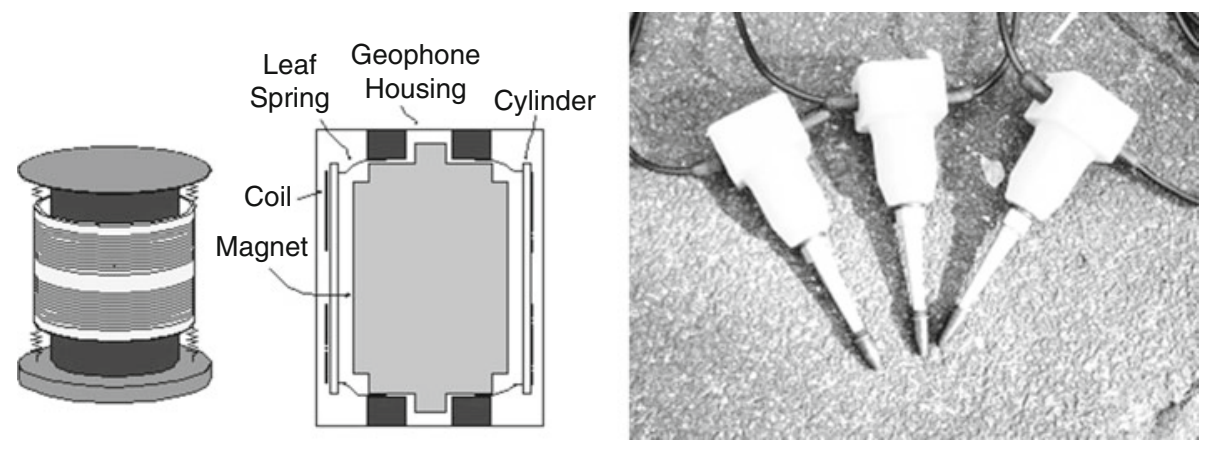

Fig. 2.24 (Left) Schematic representation of a geophone containing a permanent magnet surrounded by a coil of wire wound around a cylinder. The leaf spring has a very small stiffness making the natural frequency much lower than the frequencies of interest. The weak suspension means that the coil is effectively stationary and the magnet, attached rigidly to the case, moves with the ground. The relative motion of the coil and magnet generates the electrical signal in the same way as the electrodynamic microphone. (Right) Three commercial geophones with spikes that facilitate their insertion into the ground

mechanism (e.g., piezoelectric or electrodynamic) is contained within the sensor, it can only measure the motion of the mass relative to the motion of the case, just as was done for the vibration isolator. The solution is identical to Eqs. (2.98) and (2.99).

Such vibration sensors are called seismic sensors (seismometers) or geophones if the sensor is operated in its mass-controlled regime where $\Omega \gg 1$. For that circumstance, the mass remains nearly at rest, and the transduction mechanism senses the relative motion of the case. If the transduction mechanism measures the relative displacement, then the sensor is a seismometer. If the transduction mechanism is a coil and magnet (i.e., electrodynamic), then the sensor's output is proportional to the relative velocity of the (nearly stationary) mass and the case, and the sensor is called a geophone.

Seismometers are used to measure earthquake activity, and geophones are usually used for exploration geophysics (e.g., finding sub-surface fossil fuel deposits). Frequently, dozens of geophones will be placed in the ground, and an explosive or vibratory mechanical source will be used to create seismic waves. The outputs of the geophones' response will be processed to create images of sub-surface features. Figure 2.24 shows the typical construction of a geophone.

It is easy to control the damping of the geophone by adjusting the electrical resistance across the output of the coil. The coil's motion, relative to the magnet, will generate the voltage, $V_{\text {emf }}=(B \ell) v_{\text {coil }}$, in accordance with Faraday's law. As shown in Fig. 1.20, the coil will have some electrical resistance, $R_{\text {int }}$, but if the load resistance, $R_{\text {load }}$, is infinite, no current will flow, $I_{\text {out }}=0$, and the voltage across the coil's terminals will be $V_{\text {emf }}$.

If a load resistance is placed across the geophone's terminals, then the geophone's output voltage, $V_{\text {out }}$, will be reduced, and the current flowing through the coil and the load will be $I_{\text {out }}$, assuming the coil's inductance, $L_{\text {coil }}$, can be ignored (i.e., $\omega L_{\text {coil }} \ll\left[R_{\text {int }}+R_{\text {load }}\right]$ ).

$$
V_{\text {out }}=\frac{R_{\text {load }}}{R_{\text {int }}+R_{\text {load }}} V_{\text {emf }} \quad \text { and } \quad I_{\text {out }}=\frac{V_{\text {out }}}{R_{\text {load }}}
$$

The time-averaged power dissipated in the electrical circuit, $\left\langle\Pi_{e l}\right\rangle_{t}$, will produce an equivalent mechanical dissipation that can be represented as an equivalent mechanical resistance, $R_{e q}$. 


$$
\begin{aligned}
\left\langle\Pi_{\text {el }}\right\rangle_{t} & =\frac{V_{\text {emf }} I_{\text {out }}}{2}=\frac{1}{2} \frac{V_{\text {emf }}^{2}}{R_{\text {int }}+R_{\text {load }}}=\frac{1}{2} \frac{(B \ell)^{2} v_{\text {coil }}^{2}}{R_{\text {int }}+R_{\text {load }}}=\frac{1}{2} R_{\text {eq }} v_{\text {coil }}^{2} \\
R_{\text {eq }} & =\frac{(B \ell)^{2}}{R_{\text {int }}+R_{\text {load }}} \Rightarrow Q_{e q}=\frac{\omega_{o} m}{R_{\text {eq }}} \Rightarrow \frac{1}{Q_{\text {total }}}=\frac{R_{m}}{\omega_{o} m}+\frac{1}{Q_{\text {eq }}}
\end{aligned}
$$

A geophone that gets quite a bit of use in our laboratory is the Geo Space GS-14-L3. It is contained within a small cylindrical capsule about $17 \mathrm{~mm}$ in both height and diameter. It has a natural frequency of $f_{o}=\omega_{o} / 2 \pi \cong 28 \mathrm{~Hz}$. The coil has an internal resistance, $R_{\text {int }}=570 \Omega$, and a moving mass of $m=2.15 \mathrm{~g}$. The inductance of the coil, $L_{\text {coil }}=45 \mathrm{mH}$, so even at $1.0 \mathrm{kHz}, \omega L_{\text {coil }} / R_{\text {int }}<1 / 2$. Its output voltage as a function of frequency and electrical load resistance, $R_{\text {load }}$, is shown in Fig. 2.25.

If the vibration sensor is operated in its stiffness-controlled regime, $\Omega \ll 1$, then it is called an accelerometer. The motion of the case and the mass are nearly equal, and the transduction mechanism measures the force necessary to maintain their spacing. Two typical piezoelectric accelerometers are shown in Fig. 4.6 and are analyzed in Sect. 4.3.1.

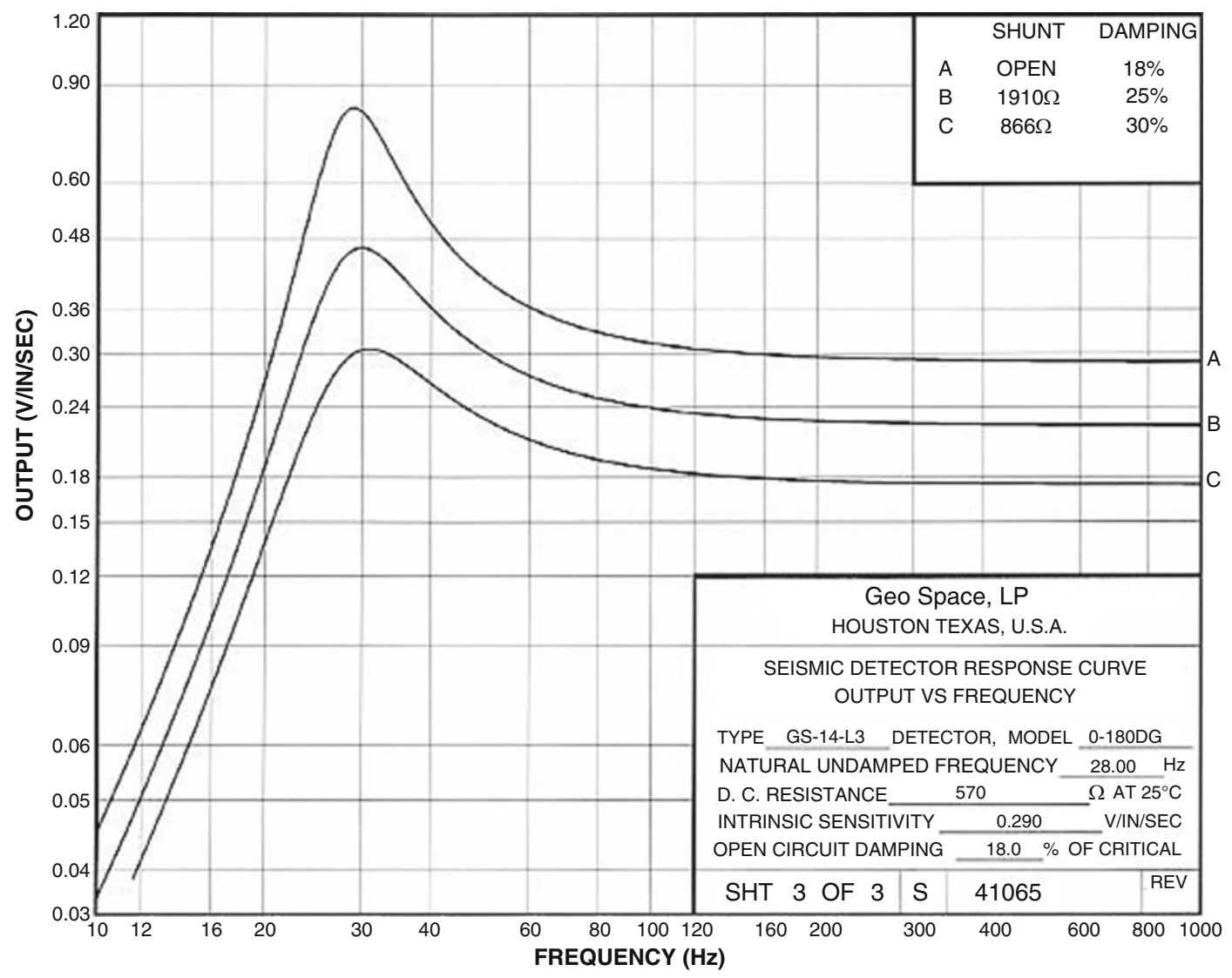

Fig. 2.25 Output sensitivity [V/(in/s)] for a Geo Space GS-14-L3 geophone capsule as a function of frequency for load resistances of $R_{\text {load }}=\infty$ (open circuit sensitivity), $R_{\text {load }}=1.91 \mathrm{k} \Omega$, and $R_{\text {load }}=866 \Omega$. The external load resistance adds damping, lowering $Q$, and reducing the sensitivity but making the sensitivity more uniform over a larger range of frequencies. (In the words of Prof. S. J. Putterman, "The flattest response is no response at all.") 


\subsection{Coupled Oscillators}

The techniques developed for our understanding and analysis of the one degree-of-freedom simple harmonic oscillators can be extended to multiple masses joined by multiple springs. This problem is important because we frequently couple one harmonic oscillator to another resonator to drive that system (which may have multiple resonances) as discussed in Sect. 10.7.5 and illustrated in Figs. 10.12 and 10.14. In fact, for closely coupled oscillators, the distinction between the driven system and the driver becomes blurred. Coupled harmonic oscillators are also an important subject because it provides a logical transition to continuous systems as the number of individual masses and springs becomes very large. Since all matter is composed of atoms, it is possible to treat solids as a three-dimensional array of masses joined to their nearest neighbors by springs.

The treatment in this section will again be restricted to motions that are constrained to occur in only one dimension, although we will let the number of degrees of freedom increase to two or more, with the number of coordinates specifying the position of the masses increasing accordingly. In general, for motion restricted to a single dimension, the number of natural frequencies for vibration will also be equal to the number of degrees of freedom, but simple harmonic motion of the coupled system will only be periodic at a single frequency if the system is excited in one of its normal modes. If those normal mode frequencies are related by the ratio of integers, then the motion of the coupled system, when disturbed from equilibrium, will be periodic; otherwise the motion will never repeat itself.

Some characteristics of the behavior of coupled systems will be unfamiliar to many readers. For that reason, we will begin our study of coupled oscillators with a very simple pair of coupled undamped oscillators that can be understood intuitively prior to developing the mathematical formalism necessary to write down solutions to the general case.

\subsubsection{Two Identical Masses with Three Identical Springs}

Thus far we have analyzed the dynamics of a single mass, joined to a spring and damper, that is either oscillating freely or driven by some externally imposed periodic force or displacement. At this point, that development has generated one-hundred numbered equations as well as many others that are contained within the text. Now that we are going to extend this analysis to several such oscillatory systems that are coupled in ways that permit them to exchange their energy of vibration, we might expect the solutions to be even more complex mathematically. That expectation will soon be fulfilled.

There is a simple case that can be understood intuitively. It will give us some basis to test the results of a more rigorous treatment of the general problem. It will also introduce simple behaviors that will be characteristic of the more general case illustrated schematically in Fig. 2.26.

We start by letting $m_{1}=m_{2}=m$ and $\mathrm{K}_{1}=\mathrm{K}_{2}=\mathrm{K}_{3}=\mathrm{K}$. We can then guess the two frequencies that will make the combined motion simple and harmonic and identify the periodic displacements of

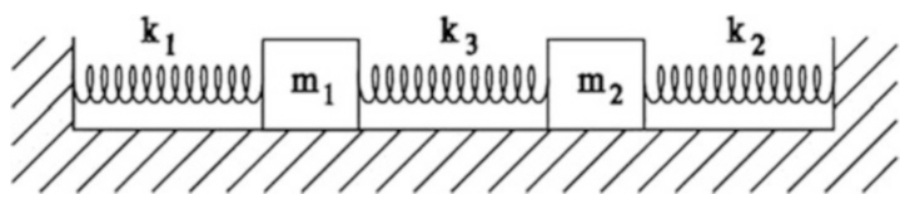

Fig. 2.26 One-dimensional, two degree-of-freedom, coupled harmonic oscillators. The $x_{1}$ coordinate measures the displacement of $m_{1}$ from its equilibrium position, and $x_{2}$ is the measure of $m_{2}$ 's displacement from equilibrium. (Figure courtesy of J. D. Maynard [23]) 
those two masses that correspond to those two frequencies of oscillation. If $x_{1}(t)=x_{2}(t)$, the motion is symmetric, and the middle spring that couples their motion, $\mathrm{K}_{3}$, is neither compressed nor extended. The frequency of oscillation is just that of either mass oscillating in isolation, $\omega_{s}=\sqrt{\mathrm{K} / m}$, where the subscript " $s$ " has been chosen because it corresponds to the symmetric mode of oscillation.

In the antisymmetric mode, the masses move $180^{\circ}$ out-of-phase with each other, $x_{1}(t)=-x_{2}(t)$. In that case, the center of the $\mathrm{K}_{3}$ spring is fixed. From the perspective of either mass, it appears as though the $\mathrm{K}_{3}$ spring has been cut in half and attached to a rigid boundary. The coupling spring, appearing half as long, is now twice as stiff (see Sect. 2.2.1), so the total effective stiffness experienced by either mass oscillating in the antisymmetric mode is $\mathrm{K}_{\mathrm{a}}=\mathrm{K}+2 \mathrm{~K}=3 \mathrm{~K}$. The frequency of that antisymmetric mode is therefore $\omega_{a}=\sqrt{3 \mathrm{~K} / m}$.

If the masses are initially displaced by either $x_{1}(t)=x_{2}(t)$ or $x_{1}(t)=-x_{2}(t)$ and then released, the pair will both oscillate at $\omega_{s}$ or $\omega_{a}$ with the same amplitudes forever. Any other combination of initial displacements will lead to motion that is entirely aperiodic; in fact, the motion, though deterministic, will appear chaotic. The reason for this lack of periodicity is that the ratio of the two normal mode frequencies is an irrational number: $\omega_{a} / \omega_{s}=\sqrt{3}$.

\subsubsection{Coupled Equations for Identical Masses and Springs}

It is not very difficult to write down the equations of motion for each mass using Newton's Second Law of Motion. It is also simple to convert those coupled differential equations to coupled algebraic equations by substituting a time-harmonic solution, just as we have been doing for our single degreeof-freedom oscillators. We will do this first for our simple case of $m_{1}=m_{2}=m$ and $\mathrm{K}_{1}=\mathrm{K}_{2}=\mathrm{K}_{3}=\mathrm{K}$.

$$
m \ddot{x}_{1}+\mathrm{K} x_{1}+\mathrm{K}\left(x_{1}-x_{2}\right)=m \ddot{x}_{1}+\mathrm{K}\left(2 x_{1}-x_{2}\right)=0
$$

By symmetry, the equation of motion for the second mass will interchange the subscripts on the coordinates.

$$
m \ddot{x}_{2}+\mathrm{K}\left(2 x_{2}-x_{1}\right)=0
$$

Since we seek normal modes, where all parts of the coupled system oscillate at the same frequency, substitution of $x_{1}=B_{1} \cos \omega t$ and $x_{2}=B_{2} \cos \omega t$ converts Eqs. (2.102) and (2.103) into two coupled algebraic equations for the two amplitude coefficients, after dividing through by $\cos \omega t$.

$$
\begin{aligned}
& -m \omega^{2} B_{1}+\mathrm{K}\left(2 B_{1}-B_{2}\right)=0 \\
& -m \omega^{2} B_{2}+\mathrm{K}\left(2 B_{2}-B_{1}\right)=0
\end{aligned}
$$

That equation can be rearranged to compute a solution for $B_{1}$ and $B_{2}$.

$$
\begin{aligned}
& \left(2 \mathrm{~K}-\omega^{2} m\right) B_{1}-\mathrm{K} B_{2}=0 \\
& -\mathrm{K} B_{1}+\left(2 \mathrm{~K}-\omega^{2} m\right) B_{2}=0
\end{aligned}
$$

For these coupled linear equations to have a nontrivial solution, the determinant of their coefficients must vanish.

$$
\left|\begin{array}{cc}
\left(2 \mathrm{~K}-\omega^{2} m\right) & -\mathrm{K} \\
-\mathrm{K} & \left(2 \mathrm{~K}-\omega^{2} m\right)
\end{array}\right|=0
$$


Evaluation of that determinant leads to a characteristic equation ${ }^{27}$ that can be solved to determine the values of $\omega$ that satisfy the original equations.

$$
\left(2 \mathrm{~K}-\omega^{2} m\right)^{2}-\mathrm{K}^{2}=0 \quad \Rightarrow \quad\left(2 \mathrm{~K}-\omega^{2} m\right)= \pm \mathrm{K}
$$

The two solutions correspond to the two modes we guessed in Sect. 2.7.1.

$$
\omega=\sqrt{\frac{2 \mathrm{~K} \pm \mathrm{K}}{m}} \quad \text { so } \quad \omega_{s}=\sqrt{\frac{\mathrm{K}}{m}} \text { and } \omega_{a}=\sqrt{\frac{3 \mathrm{~K}}{m}}
$$

Substitution of those frequencies back into either of the algebraic equations for the coefficients of $B_{1}$ and $B_{2}$ in Eq. (2.104) will provide expressions for $x_{1}(t)$ and $x_{2}(t)$ corresponding to those two normal mode frequencies, $\omega_{s}$ and $\omega_{a}$. Starting with the symmetric mode frequency, $\omega_{s}$,

$$
-m \omega_{s}^{2} B_{1}+\mathrm{K}\left(2 B_{1}-B_{2}\right)=-\mathrm{K} B_{1}+\mathrm{K}\left(2 B_{1}-B_{2}\right)=\mathrm{K}\left(B_{1}-B_{2}\right)=0 .
$$

Since $\mathrm{K} \neq 0$, the result for the symmetric mode has $B_{1}=B_{2}$. Making the similar substitution using the antisymmetric normal mode frequency $\omega_{a}$,

$$
-m \omega_{a}^{2} B_{1}+\mathrm{K}\left(2 B_{1}-B_{2}\right)=-3 \mathrm{~K} B_{1}+\mathrm{K}\left(2 B_{1}-B_{2}\right)=-\mathrm{K}\left(B_{1}+B_{2}\right)=0
$$

For the antisymmetric mode, $B_{1}=-B_{2}$.

These results will allow us to write the solution for motions of the individual masses if they are oscillating at one of those two normal mode frequencies.

$$
\begin{array}{ccc}
x_{1}(t)=B_{s} \cos \left(\omega_{s} t+\phi_{s}\right) & \text { and } & x_{2}(t)=B_{s} \cos \left(\omega_{s} t+\phi_{s}\right) \\
& \text { or } \\
x_{1}(t)=B_{a} \cos \left(\omega_{a} t+\phi_{a}\right) & \text { and } \quad x_{2}(t)=-B_{a} \cos \left(\omega_{a} t+\phi_{a}\right)
\end{array}
$$

An arbitrary phase has been introduced to accommodate any choice of the time corresponding to $t=0$.

\subsubsection{Normal Modes and Normal Coordinates}

Having identified the two frequencies for our simple case and the corresponding motions of both masses at those two frequencies, we will now see that a coordinate transformation yields further simplifications. We can introduce the new coordinates, $\eta_{s}(t)$ and $\eta_{a}(t)$, that combine the coordinates of the two masses, $x_{1}(t)$ and $x_{2}(t)$, in a way that makes $\eta_{a}(t)=0$ for the symmetric mode and $\eta_{s}(t)=0$ for the antisymmetric mode.

$$
\left.\begin{array}{l}
\eta_{s}=x_{1}+x_{2} \\
\eta_{a}=x_{1}-x_{2}
\end{array}\right\} \Rightarrow\left\{\begin{array}{l}
x_{1}=\frac{1}{2}\left(\eta_{s}+\eta_{a}\right) \\
x_{2}=\frac{1}{2}\left(\eta_{s}-\eta_{a}\right)
\end{array}\right.
$$

Those transforms allow substitution of $\eta_{a}(t)$ and $\eta_{s}(t)$ for $x_{1}(t)$ and $x_{2}(t)$ into Eq. (2.103).

\footnotetext{
${ }^{27}$ In older treatments, the characteristic equation is often called the secular equation.
} 


$$
\begin{aligned}
& \frac{1}{2}\left[m\left(\ddot{\eta}_{a}+\ddot{\eta}_{s}\right)+\mathrm{K}\left(\eta_{s}+3 \eta_{s}\right)\right]=0 \\
& \frac{1}{2}\left[m\left(\ddot{\eta}_{a}-\ddot{\eta}_{s}\right)+\mathrm{K}\left(-\eta_{s}+3 \eta_{s}\right)\right]=0
\end{aligned}
$$

Adding and subtracting the above equations from each other produces two uncoupled equations for the normal coordinates, $\eta_{s}$ and $\eta_{a}$.

$$
m \ddot{\eta}_{s}+\mathrm{K} \eta_{s}=0 \text { and } m \ddot{\eta}_{a}+3 \mathrm{~K} \eta_{a}=0
$$

The solutions for the time dependence of these normal coordinates are now trivial, since the above equations are isomorphic to our undamped simple harmonic oscillator equations.

$$
\eta_{s}(t)=C_{s} \cos \left(\omega_{s} t+\phi_{s}\right) \quad \text { and } \quad \eta_{a}(t)=C_{a} \cos \left(\omega_{a} t+\phi_{a}\right)
$$

These normal coordinates provide a complete basis that can be used to express the time dependence of the motion of both masses. The normal coordinates can express initial conditions, for example, those in Eq. (2.116), that do not correspond to initial conditions that produce the simple harmonic motion when the system is displaced from equilibrium into a configuration corresponding to one of those normal modes. Before looking at the time evolution of the positions of the masses for other initial conditions, it will be worthwhile to summarize the results of our simple example that will be present in the solutions to the more general case:

- There will be a number of normal modes that is equal to the number of degrees of freedom.

- A normal mode will have a frequency at which all of the masses will exhibit simple harmonic motion at that frequency.

- Each normal mode will provide a transformation of coordinates that can decouple the equations of motion.

- The normal modes provide a complete set of basic functions that can be combined to describe the subsequent motion of the masses for initial conditions that are not those which produce the simple harmonic oscillations characteristic of the normal modes.

\subsubsection{Other Initial Conditions}

To develop an appreciation for the behavior of our simple system of two identical masses connected by three identical springs, we can construct a solution for a very simple initial condition that does not correspond to one of the normal modes by fixing $m_{2}$ at its equilibrium position, $x_{2}(0)=0$, and displacing $m_{1}$ by an amount $x_{1}(0)=\alpha$, with both masses initially at rest, ${ }^{\prime} x_{1}(0)={ }^{\prime} x_{2}(0)=0$. Those initial conditions can be expressed in terms of the normal modes and their frequencies.

$$
x_{1}(t)=\frac{\alpha}{2} \cos \omega_{s} t+\frac{\alpha}{2} \cos \omega_{a} t \quad \text { and } \quad x_{2}(t)=\frac{\alpha}{2} \cos \omega_{s} t-\frac{\alpha}{2} \cos \omega_{a} t
$$

Upon release of both masses at $t=0$, the evolution of their positions can be interpreted by use of the following trigonometric identity: 


$$
\begin{aligned}
\cos x \pm \cos y & =\cos \left[\frac{1}{2}(x+y)+\frac{1}{2}(x-y)\right] \pm \cos \left[\frac{1}{2}(x+y)-\frac{1}{2}(x-y)\right] \\
& =\left\{\begin{array}{l}
2 \cos \frac{1}{2}(x+y) \cos \frac{1}{2}(x-y) \\
-2 \sin \frac{1}{2}(x+y) \sin \frac{1}{2}(x-y)
\end{array}\right.
\end{aligned}
$$

The ratio of the frequencies of our normal modes is an irrational number: $\omega_{a} / \omega_{s}=\sqrt{3}$. Their sum and difference can be expressed in terms of the symmetric mode frequency: $\omega_{a}+\omega_{b}=$ $(\sqrt{3}+1) \omega_{s} \cong 2.732 \omega_{s}$ and $\omega_{a}-\omega_{b}=(\sqrt{3}-1) \omega_{s} \cong 0.732 \omega_{s}$.

$$
\begin{aligned}
& x_{1}(t)=\alpha \cos \left(1.366 \omega_{s} t\right) \cos \left(0.366 \omega_{s} t\right) \\
& x_{2}(t)=-\alpha \sin \left(1.366 \omega_{s} t\right) \sin \left(0.366 \omega_{s} t\right)
\end{aligned}
$$

Although this result is deterministic, it is not periodic. As will be demonstrated for the case of weak coupling, the identity in Eq. (2.117) only leads to periodic solutions if the ratio of the frequencies is the ratio of two integers.

\subsubsection{General Solutions for Two Masses and Three Springs}

Now that the behavior of two coupled harmonic oscillators has been demonstrated for the simplest case, we can progress to the solution of the more general case where the same techniques will allow us to write equations for $m_{1} \neq m_{2}$ and $\mathrm{K}_{1} \neq \mathrm{K}_{2} \neq \mathrm{K}_{3}$. For this more general case, Eqs. (2.102) and (2.103) have to be re-written to include the features of Fig. 2.26.

$$
m_{1} \ddot{x}_{1}=-\left(\mathrm{K}_{1}+\mathrm{K}_{3}\right) x_{1}+\mathrm{K}_{3} x_{2} \quad \text { and } \quad m_{2} \ddot{x}_{2}=-\left(\mathrm{K}_{2}+\mathrm{K}_{3}\right) x_{2}+\mathrm{K}_{3} x_{1}
$$

We expect that the lighter mass will experience greater displacements than the heavier mass. The above equations of motion can be simplified if we re-scale the coordinates by introducing the new primed coordinates.

$$
x_{1}(t)=\frac{x_{1}(t)^{\prime}}{\sqrt{m_{1}}} \quad \text { and } \quad x_{2}(t)=\frac{x_{2}(t)^{\prime}}{\sqrt{m_{2}}}
$$

Substitution into Eq. (2.119) provides a pair of equations that resemble those of a driven harmonic oscillator where the coupling spring's stiffness, $K_{3}$, is proportional to the driving force due to the motion of the other mass.

$$
\frac{d^{2} x_{1}^{\prime}}{d t^{2}}+\omega_{1}^{2} x_{1}^{\prime}=\kappa^{2} x_{2}^{\prime} \quad \text { and } \quad \frac{d^{2} x_{2}^{\prime}}{d t^{2}}+\omega_{2}^{2} x_{2}^{\prime}=\kappa^{2} x_{1}^{\prime}
$$

The new frequencies which appear above are related to the individual masses and springs or to the spring which couples their motion.

$$
\omega_{1}^{2}=\frac{\left(\mathrm{K}_{1}+\mathrm{K}_{3}\right)}{m_{1}} ; \quad \omega_{2}^{2}=\frac{\left(\mathrm{K}_{2}+\mathrm{K}_{3}\right)}{m_{2}} ; \quad \kappa^{2}=\frac{\mathrm{K}_{3}}{\sqrt{m_{1} m_{2}}}
$$

If we seek the normal modes for this more general system, as before, those modes must correspond to simple harmonic motion of both masses oscillating harmonically at the same frequency. For that reason, we can substitute harmonic solutions of the same frequency, $\omega$, for $x_{1}^{\prime}(t)=\mathfrak{R e}\left[\widehat{\mathbf{x}}_{\mathbf{1}}^{\prime} e^{j \omega t}\right]$ and 
$x_{2}^{\prime}(t)=\mathfrak{R e}\left[\widehat{\mathbf{x}}_{2}^{\prime} e^{j \omega t}\right]$. This substitution again converts the coupled differential expressions in Eq. (2.121) to coupled algebraic equations.

$$
\begin{gathered}
\left(\omega_{1}^{2}-\omega^{2}\right) x_{1}^{\prime}-\kappa^{2} x_{2}^{\prime}=0 \\
-\kappa^{2} x_{1}^{\prime}+\left(\omega_{2}^{2}-\omega^{2}\right) x_{2}^{\prime}=0
\end{gathered}
$$

As before, we impose the requirement that the determinant of the coefficients for the coupled equations vanish so that nontrivial solutions can be identified.

$$
\left|\begin{array}{cc}
\left(\omega_{1}^{2}-\omega^{2}\right) & -\kappa^{2} \\
-\kappa^{2} & \left(\omega_{2}^{2}-\omega^{2}\right)
\end{array}\right|=0
$$

Evaluation of the determinant results in a secular (characteristic) equation for the frequencies which is biquadratic.

$$
\omega^{4}-\left(\omega_{1}^{2}+\omega_{2}^{2}\right) \omega^{2}+\omega_{1}^{2} \omega_{2}^{2}-\kappa^{4}=0
$$

The solution can be obtained using the quadratic formula to determine $\omega^{2}$ in terms of the oscillators' parameters as defined in Eq. (2.122).

$$
\begin{aligned}
\omega & =\left[\frac{1}{2}\left(\omega_{1}^{2}+\omega_{2}^{2}\right) \pm \frac{1}{2} \sqrt{\left(\omega_{1}^{2}-\omega_{2}^{2}\right)^{2}+4 \kappa^{4}}\right]^{1 / 2} \\
& =\frac{1}{\sqrt{m_{1} m_{2}}}\left[\frac{1}{2}\left[\left(\mathrm{~K}_{1}+\mathrm{K}_{3}\right) m_{2}+\left(\mathrm{K}_{2}+\mathrm{K}_{3}\right) m_{1}\right] \pm \frac{1}{2} \sqrt{\left[\left(\mathrm{K}_{1}+\mathrm{K}_{3}\right) m_{2}-\left(\mathrm{K}_{2}+\mathrm{K}_{3}\right) m_{1}\right]^{2}+4 \mathrm{~K}_{3}^{2} m_{1} m_{2}}\right]^{1 / 2}
\end{aligned}
$$

As before, we have identified the two unique frequencies that allow both masses to oscillate harmonically at the same frequency. If those frequencies are substituted back into the algebraic expressions of Eq. (2.123), the ratios of $x_{1}^{\prime}$ to $x_{2}^{\prime}$ can be determined.

Since the masses and springs are now different, the normal mode displacements will be more complex than the simple solutions of Sect. 2.7.2. The normal mode descriptions for $x_{1}(t)$ and $x_{2}(t)$ will still be classified as symmetric and antisymmetric, but the amplitudes will no longer be identical. The reader is referred to the treatment by Morse for those solutions in their full algebraic ugliness [33].

\subsubsection{Driven Oscillators, Level Repulsion, and Beating}

In the limit that $\mathrm{K}_{3}=0$, so that there is no coupling between the two masses, it is comforting that Eq. (2.126) regenerates the frequencies of the two isolated oscillators. It is instructive to consider the case of two oscillators of the same frequency, $\omega_{1}=\omega_{2}$, that are weakly coupled so that $\kappa \ll \omega_{1}$. Under those circumstances, we can use a Taylor series to expand Eq. (2.126) and provide the symmetric and antisymmetric modal frequencies.

$$
\begin{aligned}
& \omega_{s}=\sqrt{\omega_{1}^{2}-\kappa^{2}}=\omega_{1} \sqrt{1-\frac{\kappa^{2}}{\omega_{1}^{2}}} \cong \omega_{1}-\frac{\kappa^{2}}{2 \omega_{1}} \\
& \omega_{a}=\sqrt{\omega_{1}^{2}+\kappa^{2}}=\omega_{1} \sqrt{1+\frac{\kappa^{2}}{\omega_{1}^{2}}} \cong \omega_{1}+\frac{\kappa^{2}}{2 \omega_{1}}
\end{aligned}
$$


The fact that even weak coupling results in a shift of the natural frequencies of the two uncoupled oscillators is known as level repulsion. That term is motived by analogy to splitting of energy levels in atomic systems when such levels become coupled, possibly by electromagnetic interactions. ${ }^{28}$ At first glance, the symmetric frequency, $\omega_{s}$, should not be shifted, based on the normal modes of the simpler system of equal masses and springs analyzed in Sects. 2.7.1 and 2.7.2. The fact that $\omega_{s}$ is downshifted is a consequence of the assumption that the motion of our simpler system was initiated by making two identical displacements. If one of the masses is driven, then the coupling spring must be displaced initially to permit the transfer of energy from the driven mass to the other mass.

It is easy to visualize this periodic energy transfer in the weak-coupling limit, with $\omega_{s} \cong \omega_{a}$, and $n$ $\left(\omega_{s}-\omega_{a}\right)=\left(\omega_{s}+\omega_{a}\right)$, where $n$ is chosen to be an integer. Using the identities of Eq. (2.117), and assuming the oscillations start with $x_{1}(0)=\alpha$ and $x_{2}(0)=0$, the subsequent evolution of $x_{1}(t)$ and $x_{2}(t)$ can be written as the product of two trigonometric functions.

$$
\begin{aligned}
& x_{1}(t)=\alpha\left[\cos \frac{\left(\omega_{a}-\omega_{s}\right)}{2} t\right]\left[\cos \frac{\left(\omega_{a}+\omega_{s}\right)}{2} t\right] \\
& x_{2}(t)=\alpha\left[\sin \frac{\left(\omega_{a}-\omega_{s}\right)}{2} t\right]\left[\sin \frac{\left(\omega_{a}+\omega_{s}\right)}{2} t\right]
\end{aligned}
$$

The second term in both products represents a simple harmonic oscillation at a frequency which is the average of $\omega_{a}$ and $\omega_{s}$. The first terms define an amplitude modulation envelope that closes (i.e., goes to zero) with a period that is integer multiples of $\pi /\left(\omega_{a}-\omega_{s}\right)$. Since the peaks in the envelopes for $x_{1}$ and $x_{2}$ are $90^{\circ}$ out-of-phase, it is apparent that all of the energy starts in $m_{1}$, but at a time $\pi / 2\left(\omega_{a}-\omega_{s}\right), m_{1}$ has come to rest, and all of the energy has been concentrated in $m_{2}$, with the situation reversing itself with a period $\pi /\left(\omega_{a}-\omega_{s}\right)$. This periodic modulation is known as beating and is illustrated in Fig. 2.27, where $n=12$.

Fig. 2.27 Time evolution of two weakly coupled oscillators, described by Eq. (2.128), is plotted to illustrate the periodic exchange of energy. The upper plot is $x_{1}(t)$ and the lower is $x_{2}(t)$. In this figure, the oscillation frequency $\left(\omega_{a}+\omega_{s}\right) / 2$ is 12 times the frequency difference $\left(\omega_{a}-\omega_{s}\right)$. Initially, all of the motion is in $m_{1}$
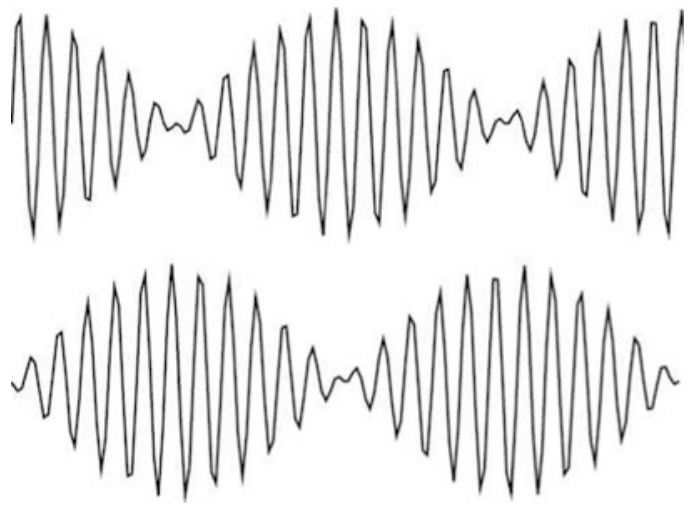

${ }^{28}$ As mentioned in Sect. 2.3.3, the atomic energy levels are related to the frequencies of light radiated during a level transition through Planck's equation: $E / \omega=\hbar$. 


\subsubsection{String of Pearls}

We can now extend this analysis of coupled oscillators to more than two masses. To make it easier to visualize the motion of several coupled masses, we will first solve the problem of an undamped, single degree-of-freedom oscillator consisting of a single mass suspended at the center of a taught string. The location of the mass on the string will be specified by the $x$ axis, and the displacement of the mass from its position of static equilibrium will be given by its $y$ coordinate. A point mass, $m$, is shown in Fig. 2.28, attached to the center of a limp string ${ }^{29}$ of length $L=2 a$, and is subject to a tension, T. Both ends of the string are immobilized: $y(0)=y(2 a)=0$. If the mass is displaced from equilibrium by a distance, $y_{1}$, at the center of the string, there will be a net force on that mass that will be opposite to $y$; hence, the mass will obey Hooke's law. We assume that the string is massless, $m_{s}=0$, and that the tension in the string has a value, $\mathrm{T}$.

It is easy to demonstrate that tension, $\mathrm{T}$, in the string exerts a linear restoring force on the mass if the vertical displacement from equilibrium, $y_{1}$, is small enough that $y_{1} / a \ll 1$. As shown in Fig. 2.28, a displacement, $y_{1}$, creates an angle, $\theta=\tan ^{-1}\left(y_{1} / a\right)$, between the displaced mass and the former equilibrium position of the string. If the displacement, $y_{1}$, is small, the change in the length of the string $\delta L$ will be second-order in the displacement, $y_{1}$, so the increase in tension due to the stretching of the string can be ignored in a first-order (Hooke's law) analysis.

$$
\delta L=2 \sqrt{\left(a^{2}+y_{1}^{2}\right)^{1 / 2}-a^{2}}=2 a \sqrt{\left(1+\frac{y_{1}^{2}}{a^{2}}\right)^{1 / 2}-1} \cong a\left(\frac{y_{1}}{a}\right)^{2}
$$

The restoring force will be linear in the displacement for small enough displacements that $\theta=\tan ^{-1}\left(y_{1} / a\right) \cong y_{1} / a$. From Fig. 2.28, Garrett's First Law of Geometry can be used to calculate the vertical component of the force, $F_{v}$, due to the tension, T, of the string.

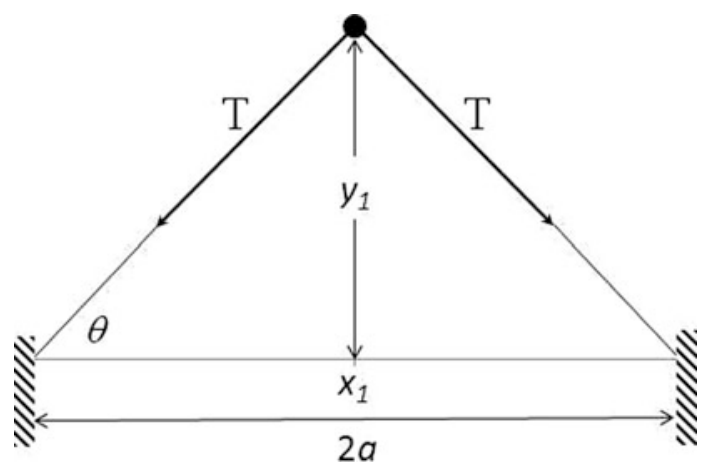

Fig. 2.28 A limp, massless string of length, $L=2 a$, is under tension, $\mathrm{T}$, and has a point mass, $m$, attached at its center and is fixed at both ends. The mass has been displaced from its equilibrium position by a distance $y_{1}$, so the string makes an angle, $\theta=\tan ^{-1}\left(y_{1} / a\right)$, with the horizontal. The two vertical components of the tension provide a linear restoring force: $F_{y}=-2 \mathrm{~T} \sin \theta \cong-(2 \mathrm{~T} / a) y_{1}$

\footnotetext{
${ }^{29}$ For this case, we will assume that the string has no flexural rigidity and that the restoring force is entirely due to the tension applied to the string. In Sect. 5.5, we will examine the stiff string, which is under tension but also exhibits flexural rigidity.
} 


$$
F_{v}=-2 \mathrm{~T} \sin \theta \cong-\left(\frac{2 \mathrm{~T}}{a}\right) y_{1}
$$

This "mass on a string" is another example of a simple harmonic oscillator that moves in one dimension, along the $y$ axis, and has an effective stiffness constant, $\mathrm{K}_{\text {eff }}=(2 \mathrm{~T} / a)$. To demonstrate the utility of this configuration for the representation of coupled oscillators, Fig. 2.29 duplicates the normal mode displacements for masses and springs from Eq. (2.111).

In Fig. 2.30, we extend this mass and string model to multiple masses. At equilibrium, each mass is separated from its adjacent masses by a horizontal distance, $a$. The position of the $i$ th mass is $x_{i}=a i$, where $1 \leq i \leq N$. For $N$ masses, the total length of the string is $L=(N+1) a$. Since the string is rigidly fixed at both ends, $y(0)=y\left(x_{N+1}\right)=0$.

The vertical component of the force, $F_{i}$, on any mass, $m_{i}$, depends only upon the position of that mass with respect to its nearest neighbors.

$$
F_{i}=\mathrm{T}(\sin \phi-\sin \theta) \cong \mathrm{T}\left(\frac{y_{i+1}-y_{i}}{a}-\frac{y_{i}-y_{i-1}}{a}\right)=-\frac{2 \mathrm{~T}}{a} y_{i}+\frac{\mathrm{T}}{a}\left(y_{i+1}+y_{i-1}\right)
$$
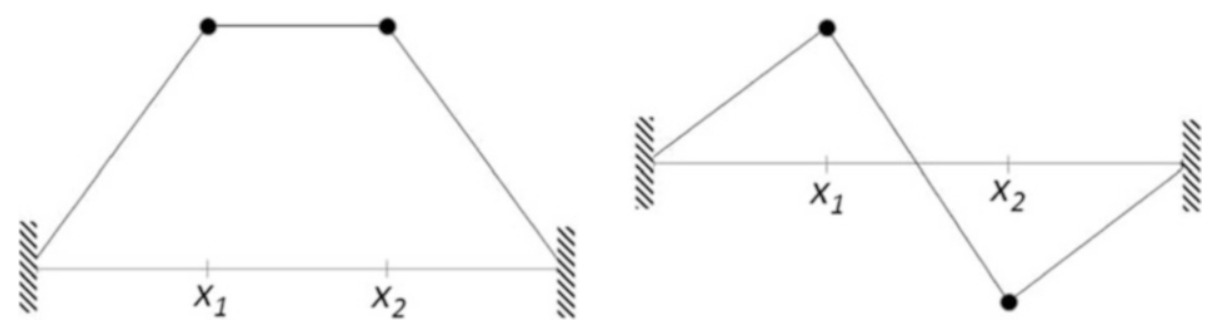

Fig. 2.29 The same two normal modes for the simple coupled oscillators with two identical masses and three identical springs that were calculated in Eq. (2.111) are reproduced here as two masses on a tensioned string. The symmetric mode is shown on the left, and the antisymmetric mode, with frequency that is $\sqrt{3}$ greater than the symmetric mode, is shown at the right
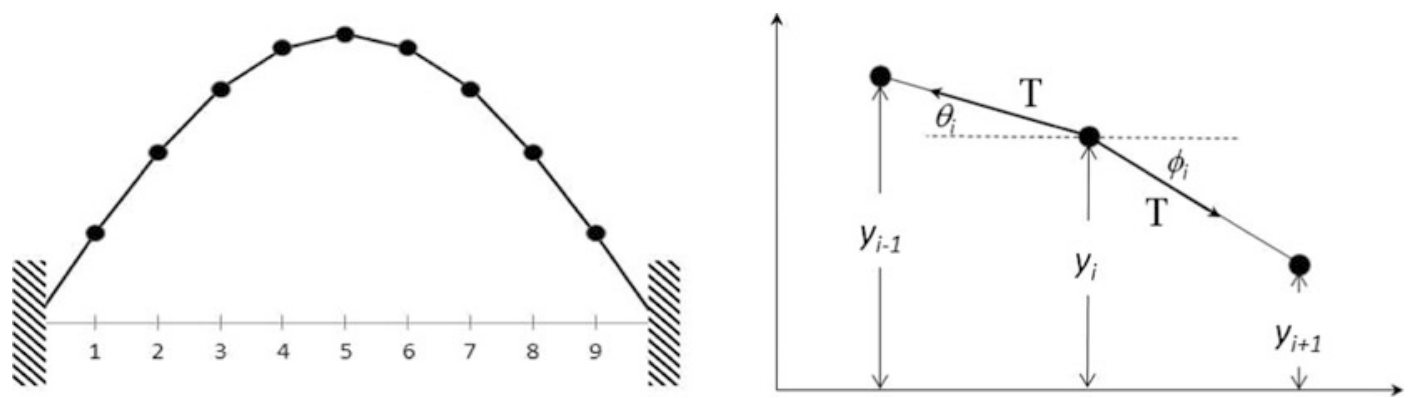

Fig. 2.30 (Left) $N=9$ masses are spaced uniformly on a string of length, $L=(N+1) a$. The coordinate of each mass is specified by its position along the $x$ axis when the string is at its equilibrium position. Each mass is constrained to move only in the vertical direction, and each mass is displaced from its equilibrium position by an amount $y_{i}$. (Right) The net vertical force on each mass, $m_{i}$, is determined by the vertical displacements of its nearest neighbors, $y_{i-1}$ and $y_{i+1}$, relative to the displacement, $y_{i}$. The vertical component of the force on the $i$ th mass is determined by the tension in the string, $\mathrm{T}$, and by the angles $\theta_{i}=\tan ^{-1}\left[\left(y_{i}-y_{i-1}\right) / a\right]$ and $\phi_{i}=\tan ^{-1}\left[\left(y_{i+1}-y_{i}\right) / a\right]$ 
If we assume all masses are equal, $m_{i}=m$, and we apply Newton's Second Law of Motion to the $i$ th mass, abbreviating (T/a) as $\mathrm{K}$, the dynamical equations describing the vertical motion of each of the $N$ masses can be written in a compact form.

$$
m \ddot{y}_{i}+2 \mathrm{~K} y_{i}-\mathrm{K}\left(y_{i+1}+y_{i-1}\right)=0 \quad \text { for } \quad i=1,2, \ldots, N
$$

This set of coupled differential equations could be solved by the techniques used previously in Sects. 2.7.3 and 2.7.5. If we define $\lambda \equiv 2-\left(m \omega^{2} a / \mathrm{T}\right)$, then the $N \times N$ determinant of the coefficients, $D_{N}$, for the algebraic equations, which resulted from the assumption that all masses vibrating in any normal mode must oscillate at the same frequency, $\omega$, has the following form that is characteristic of nearest-neighbor interactions:

$$
D_{N}=\left|\begin{array}{ccccccc}
\lambda & -1 & 0 & 0 & 0 & \cdots & 0 \\
-1 & \lambda & -1 & 0 & 0 & \cdots & 0 \\
0 & -1 & \lambda & -1 & 0 & \cdots & 0 \\
\vdots & \vdots & \vdots & \vdots & \vdots & \vdots & \vdots \\
0 & 0 & 0 & 0 & 0 & \lambda & -1 \\
0 & 0 & 0 & 0 & 0 & -1 & \lambda
\end{array}\right|
$$

Fetter and Walecka solve this system of linear equations by development of a recursion relation for the minors [34], but we will take a different approach that anticipates our transition to continuous systems in the next chapter.

Since we have chosen our masses to be equal and to be equally spaced from each other along the string, it is possible to treat the vertical displacements of the masses as a function of the $x$ coordinate: $y_{i}=y\left(x_{i}\right)$, where $x_{i}=a i$. Again, we seek normal mode solutions that require that every mass oscillates with the same frequency, $\omega$, but now we will keep track of the amplitudes of vibration of the individual masses by introducing a constant, $\beta$, which has the units of inverse length $\left[\mathrm{m}^{-1}\right]$.

$$
y\left(x_{i}, t\right)=A e^{j\left(\beta x_{i}-\omega t\right)}
$$

Substitution of that expression for displacements into Eq. (2.132) produces the algebraic Eq. (2.135) after cancellation of common exponential time factors.

$$
-m \omega^{2}+2 \mathrm{~K}-\mathrm{K}\left(e^{j \beta a}+e^{-j \beta a}\right)=0
$$

The resulting equation can be solved for the normal mode frequencies and simplified by use of the trigonometric identity, $2 \sin ^{2}(x / 2)=(1-\cos x)$, where $2 \cos x=\left(e^{j x}+e^{-j x}\right)$.

$$
\omega^{2}=\frac{2 \mathrm{~K}}{m}(1-\cos \beta a)=\frac{4 \mathrm{~K}}{m} \sin ^{2}\left(\frac{\beta a}{2}\right)
$$

Although the frequencies, $\omega$, are a continuous function of $\beta$, the normal mode frequencies will become discrete when we impose the end conditions that $y(0)=y\left(x_{N+1}\right)=0$. To satisfy the condition at $x=0$, we will subtract a solution with the form of Eq. (2.134), but with a negative $\beta$, since both the $\beta$ and $-\beta$ parts will be zero when multiplied by $x=0$.

$$
y\left(x_{i}, t\right)=\mathfrak{R e}\left[A\left(e^{j \beta x_{i}-j \omega t}-e^{-j \beta x_{i}-j \omega t}\right)\right]
$$

That form of Eq. (2.134) is clearly zero for $x_{o}=0$. For $x_{N+1}$, making $y_{N+1}=y[(N+1) a]=0$ results in a condition that discretizes the values of $\beta$, simultaneously satisfying both boundary conditions. 


$$
e^{j \beta(N+1) a}-e^{-j \beta(N+1) a}=2 j[\sin \beta(N+1) a]=0
$$

Only discrete values of $\beta_{n}$ are now acceptable.

$$
\beta_{n}(N+1) a=n \pi \quad \Rightarrow \quad \beta_{n}=\frac{n \pi}{a(N+1)} ; \quad n=1,2,3, \ldots, N
$$

Substitution of the quantized values of $\beta_{n}$ into Eq. (2.136) provides the normal mode frequencies.

$$
\omega_{n}=2 \sqrt{\frac{k}{m} \sin ^{2}\left(\frac{\beta_{n} a}{2}\right)}
$$

Plugging the $\beta_{n}$ back into Eq. (2.134) provides the time-dependent displacements for each mass.

$$
y\left(x_{i}, t\right)=y_{i}(t)=A_{n} \sin \left(\frac{i n \pi}{N+1}\right) \sin \omega_{n} t
$$

This equation provides $N$ solutions for each mass corresponding to the $n$th normal mode with normal mode frequency, $\omega_{n}$. Each normal mode has an amplitude, $A_{n}$, that would depend upon the strength of the excitation of the $n$th mode. The vertical displacements of each mass for each mode are exaggerated for all nine modes in Fig. 2.31, with $N=9$ and $A_{n}=1$ for all $n$. The ratio of the frequency of each normal mode to the frequency of the $n=1$ mode, $\omega_{n} / \omega_{1}$, is tabulated and plotted as a function of mode number, $n$, in Fig. 2.32.

The lowest-frequency modes correspond to in-phase motion of adjacent masses. The highestfrequency mode, $n=N$, corresponds to out-of-phase motion of the adjacent masses. By increasing the number of masses and decreasing the spacing to hold the overall length constant, $L=(N+1) a$, the number of modes becomes infinite, and the string with masses attached has a uniform linear mass density, $\rho_{L}=(m / a)$. The modal frequencies are those given by Eq. (2.142) in this limit.

$$
\lim _{N \rightarrow \infty} \omega_{n}^{2}=\frac{4 \mathrm{~T}}{m a}\left[\frac{n \pi}{2(N+1)}\right]^{2}=\frac{\mathrm{T}}{\rho_{L}}\left(\frac{n \pi}{L}\right)^{2}
$$

The quantity $\left(\mathrm{T} / \rho_{L}\right)^{1 / 2} \equiv c$ (for celerity) has the dimensions of speed $[\mathrm{m} / \mathrm{s}]$. As we will see when we transition to continuous systems in the next chapter, $c$ is just the speed of transverse wave propagating on a uniform string. The low-frequency normal mode frequencies, $f_{n}=\omega_{n} / 2 \pi$, correspond to integer numbers, $n$, of half-wavelengths fitting between the fixed ends.

For the discrete case with $N=9$, it is easy to imagine that the lowest-frequency mode at the top left in Fig. 2.31 approximates a half-sine waveform fit between the two fixed ends, i.e., $\lambda_{1}=2 L$, and the second mode corresponds to two half-wavelengths (i.e., one entire wavelength) between the fixed ends, $\lambda_{2}=L$.

\subsection{The Not-So-Simple (?) Harmonic Oscillator}

After 60 pages of discussion, 32 figures, and over 140 numbered display equations, the reader would be justified in requesting an explanation for why "simple" is used as a descriptor for the harmonic oscillator. The mass and the spring that are shown to function in its quasi-static limit are simple components. The description of the time history of the motion of a mass connected to a spring involves only simple trigonometric functions with constants that could be fit to specific initial conditions. The expressions for the kinetic energy stored by the mass, $K E=(1 / 2) m v^{2}$, and the potential energy stored by 

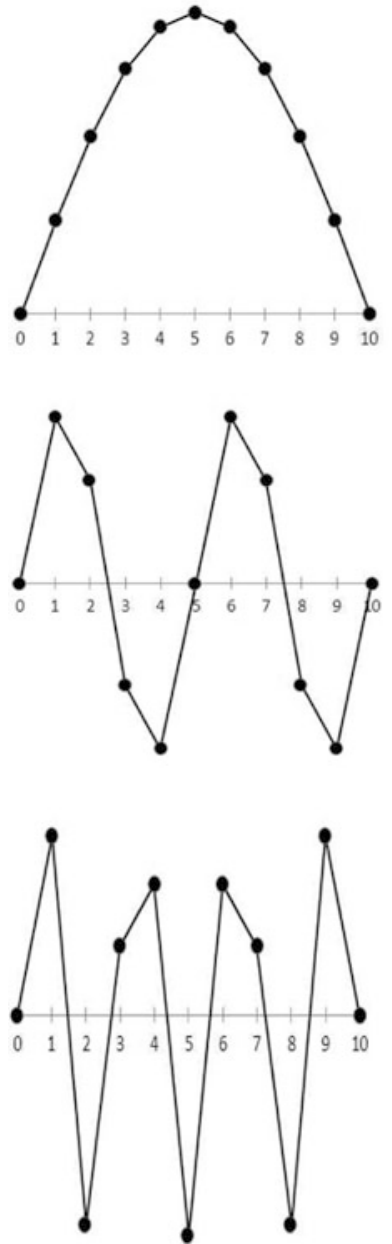
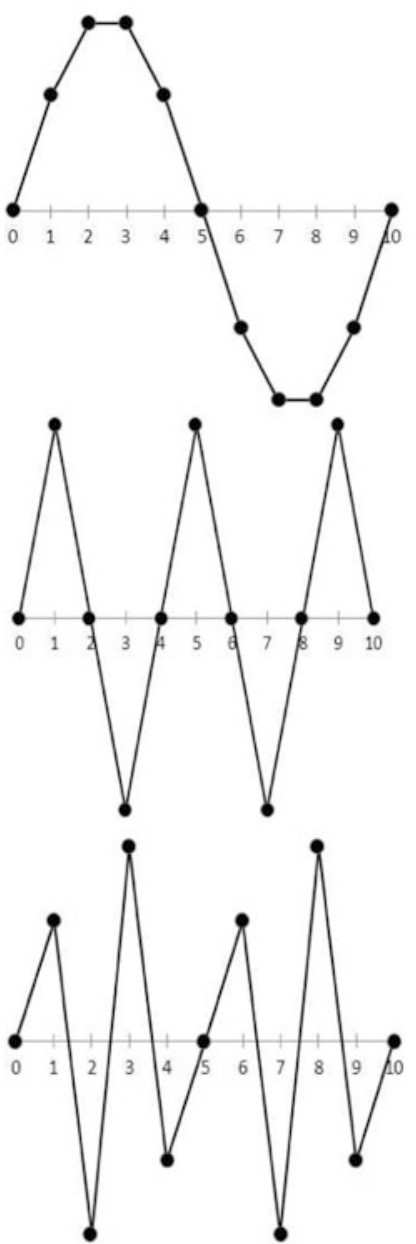
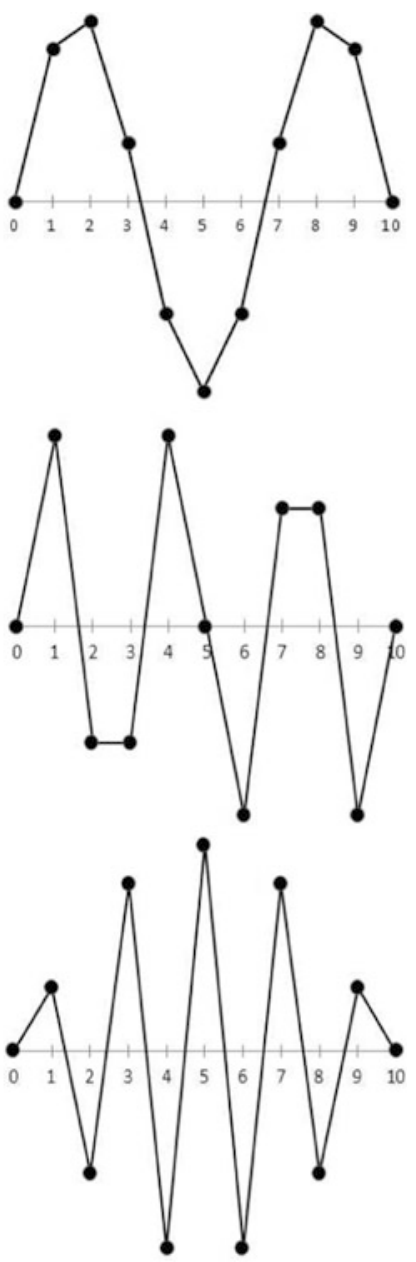

Fig. 2.31 Mode shapes for the normal modes for the nine equal masses (solid circles) on a taught string (straight lines). The solid circles at $N=0$ and $N=10$ represent the fixed ends of the string. The amplitudes are greatly exaggerated for clarity. Please remember these results are only valid for $y \ll a$. The number of zero-crossings increases with increasing mode number. The fundamental $(n=1)$ mode has no zero-crossings, and each subsequent mode has a number of zerocrossings that is one less than the mode number, $n$. The frequencies of these normal modes are tabulated and plotted in Fig. 2.32

Fig. 2.32 The normalized modal frequencies, $\omega_{n}$, based on Eq. (2.140), for nine equal masses, are tabulated at the left and plotted vs. mode number, $n$, at the right

\begin{tabular}{cc} 
Mode & Freq. \\
\hline \hline 1 & 1.000 \\
2 & 1.975 \\
3 & 2.902 \\
4 & 3.757 \\
5 & 4.520 \\
6 & 5.172 \\
7 & 5.696 \\
8 & 6.080 \\
9 & 6.238
\end{tabular}

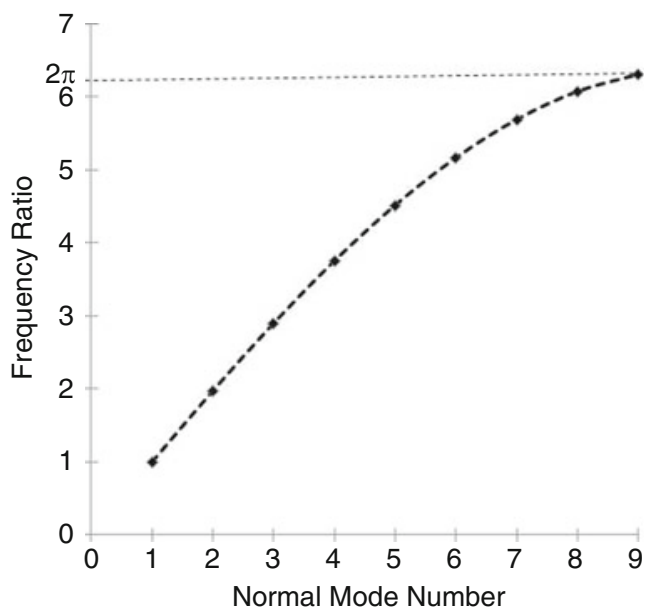


the spring, $P E=(1 / 2) \mathrm{K}^{2}$, are equally simple, and a gravitational offset had no influence on the dynamical behavior. Even Rayleigh's exploitation of the virial theorem for calculation of oscillatory frequencies would have to be judged "simple," at least in hindsight.

Once the mechanical resistance was added as a means to dissipate energy, the analysis of the oscillator's behavior left the purely mechanical perspective and entered the realm of thermodynamics the dissipated energy had to leave "the system" and make contact with "the environment." Although such calculations may be unfamiliar, their result incorporated the Equipartition Theorem, which is also quite simple - each quadratic degree of freedom, on average, gets its $(1 / 2) k_{B} T$ share of energy at thermal equilibrium. The steady-state response to systematic injection of energy into the oscillator, by either forcing the mass or jiggling the attachment point of the spring, turned out to be determined entirely by the oscillator's mechanical impedance. The approach to steady state involved addition of solutions to the freely decaying oscillator.

The experimental determination of the stiffness and moving mass for the electrodynamic loudspeaker example was achieved by simply adding some pocket change (i.e., nickels) and recording the frequency shift due to the mass loading. The mechanical resistance was determined by the free-decay time. All three parameters were determined simply to an accuracy of better than $\pm 1 \%$ by least-squares fitting, while the force factor (i.e., the $B \ell$-product that determined the force produced by the electrical current flowing in the voice coil) required only a DC electrical resistance measurement and an electrical impedance measurement at the resonance frequency.

When two or more (undamped) oscillators were coupled together, the mathematical description became more complicated, but the simplest case could be analyzed intuitively, based on the previous understanding of individual harmonic oscillators. The identification of the normal modes and their frequencies could be algebraically messy when different masses are joined by springs with different stiffnesses. The time histories of the motion might appear chaotic if the normal mode frequencies were not commensurate (i.e., related by the ratio of integers). One must admit those systems constitute a fairly complicated combinations of otherwise simple harmonic oscillators. Although the coupled solutions were the most complicated results derived in this chapter, the next chapter will simplify the situation once again by treating large numbers of coupled oscillators as a continuum, so an overall "shape" is described instead of the individual displacements of point masses.

\section{Talk Like an Acoustician}

$\begin{array}{ll}\text { Point-mass } & \text { Mechanical reactance } \\ \text { Dynamical equation } & \text { Resistance-controlled regime } \\ \text { Equation of state } & \text { Stiffness-controlled regime } \\ \text { Frequency } & \text { Mass-controlled regime } \\ \text { Period } & \text { Rayleigh line shape } \\ \text { Angular frequency } & \text { Lenz's law } \\ \text { Radian frequency } & \text { Faraday's law of induction } \\ \text { Quasi-static approximation } & \text { Decibel } \\ \text { Virial theorem } & \text { Phase-locked loop } \\ \text { Rayleigh's method } & \text { Ring down } \\ \text { Adiabatic invariance } & \text { Reversible transducer } \\ \text { Dashpot } & \text { Vibration isolator } \\ \text { Mechanical resistance } & \text { Transmissibility } \\ \text { Quality factor } & \text { Seismometer } \\ \text { Logarithmic decrement } & \text { Geophone } \\ \text { Natural frequency } & \text { Normal modes }\end{array}$


Free-decay frequency

Underdamped

Overdamped

Critical damping

Equipartition Theorem

Half-power bandwidth

Full width at half maximum

Equivalent noise bandwidth

Effective bandwidth

Mechanical impedance

Resonance
Normal mode frequencies

Symmetric mode

Antisymmetric mode

Characteristic equation

Secular equation

Normal coordinates

Basis functions

Level repulsion

Beating

Nearest-neighbor interactions

Celerity

\section{Exercises}

Each problem that I solved became a rule which served afterwards to solve other problems. (Rene Descartes)

1. U-tube oscillations. The tube shown in Fig. 2.33 is filled with a liquid that has a uniform mass density, $\rho$. The total length of the fluid column is $L$, and the cross-sectional area of the tube, $S$, is constant. When the fluid is displaced from its equilibrium position by a distance, $x$ (as shown), the column of liquid executes simple harmonic motion under the influence of a constant gravitational acceleration, $g$.

(a) Period. Calculate the period, $T$, of the oscillations, neglecting damping.

(b) Quality factor. If the oscillations are observed to decrease by $20 \%$ during each cycle, calculate $Q$.

2. Coefficient of friction oscillator. Two cylindrical rollers are located a distance $2 a$ apart. Their bearings are anchored. The rollers rotate at an angular speed $\omega_{r}$ in opposite directions, as shown in Fig. 2.34. On the top of the rollers rests a bar of length, $l$, and weight, $W=m g$.

Fig. 2.33 Liquid-filled

U-tube

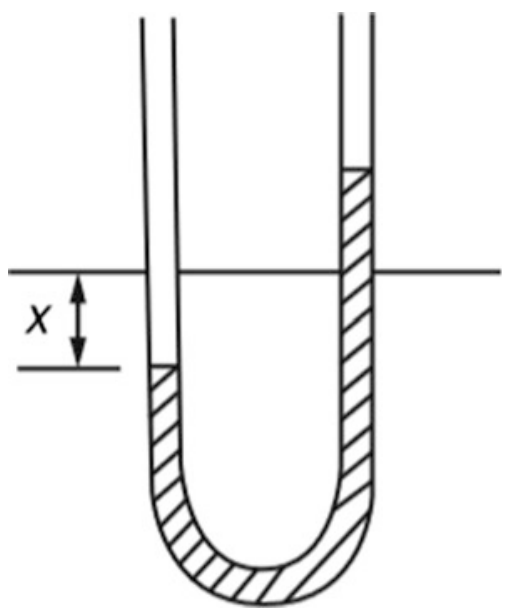


Fig. 2.34 Bar supported on two counter-rotating wheels with centers separated by $2 a$

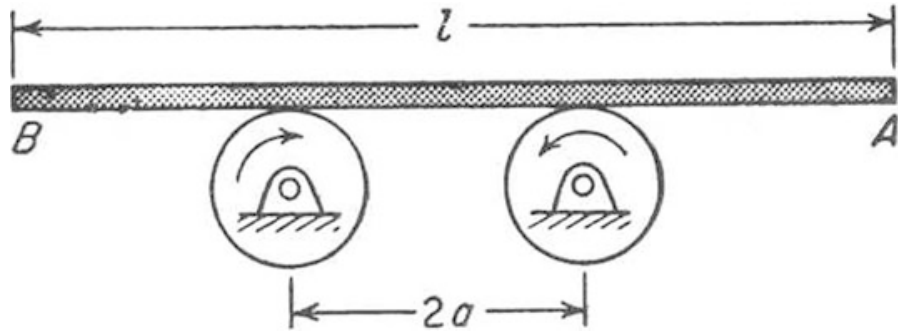

Assuming a dry coefficient-of-friction $\mu$ between the rollers and the bar, the bar will oscillate back and forth longitudinally executing simple harmonic motion. ${ }^{30}$ The restoring force will be the difference between the frictional forces in the $x$ direction, $F_{y}$ (along the bar), due to the weight (normal force) of the bar in the $y$ direction, $F_{y}=\mu m g$, of the bar on each roller. (Note that if the end of the bar is closer to one roller, the weight of the bar on the other roller will be larger and will push the bar back toward the center.) Calculate the frequency of the bar's oscillations when the wheels rotate as shown in Fig. 2.34.

3. Mass loading frequency shift. A mass-spring system is observed to have a natural frequency of $10.0 \mathrm{~Hz}$. When a mass of 10.0 grams is added to the original mass, $m_{o}$, the frequency decreases to $8.0 \mathrm{~Hz}$. Determine the original mass and the spring's stiffness constant, K.

4. Loudspeaker in an enclosure. A loudspeaker has a free cone resonance frequency of $55.5 \mathrm{~Hz}$. If a US nickel (mass $=5.00 \mathrm{~g}$ ) is placed on the speaker's dust cap, the resonance frequency is reduced to 46.3 Hz. After the nickel is removed, the speaker is installed in a sealed enclosure, and the frequency increases to $80.0 \mathrm{~Hz}$. What is the equivalent stiffness (in Newtons/meter) provided by the elasticity of the air contained within the loudspeaker enclosure?

5. Spar buoy oscillations. An oceanographic instrument package is placed at the bottom of a spar buoy shown in Fig. 2.35. The mass (in air) of the entire buoy (instruments, batteries, tube, and antenna) is $150 \mathrm{~kg}$. The tubular portion ( $5 \mathrm{~m}$ long and $25 \mathrm{~cm}$ diameter) is partially submerged. You may assume the density of the water, $\rho=1000 \mathrm{~kg} / \mathrm{m}^{3}$, and the acceleration due to gravity, $g=9.8 \mathrm{~m} / \mathrm{s}^{2}$.

(a) Equilibrium depth. How far from the surface of the water is the top of the $5.0 \mathrm{~m}$ long tubular section when it is in equilibrium (at rest)?

(b) Frequency. What is the natural frequency of vibration of the buoy if it is displaced (vertically) from its equilibrium position and released?

(c) Damping. When the buoy is displaced from equilibrium, the oscillations decay to $1 / e$ of their initial value in $30 \mathrm{~s}$. Determine the viscous (mechanical) resistance, $R_{m}$, which the water provides to damp the oscillations.

(d) Hydrodynamic mass correction. The water increases the buoy's inertia by adding a mass that is about half that of a sphere, with density $\rho$ (see Sect. 12.5.1) and with the same diameter as the buoy. Recalculate your result for the frequency from part (b) including the effective mass of the water that is driven by the motion of the cylinder.

(e) Relative motion. Assume that there is a "ring" drawn at the static (calm) water level on the tubular section at the normal equilibrium position which you calculated in part (a) of this problem. If a sinusoidal swell with peak-to-trough amplitude of $1.0 \mathrm{~m}$ passes the buoy position every 20 s (i.e., a wave with a 20 s period), what is the greatest (peak) distance which the ring

\footnotetext{
${ }^{30}$ The operation of such an oscillator is provided by Dan Russell in a YouTube video: http://www.youtube.com/watch? $\mathrm{v}=46 \mathrm{k} 2 \mathrm{FXzwT} 8$
} 
Fig. 2.35 Spar buoy

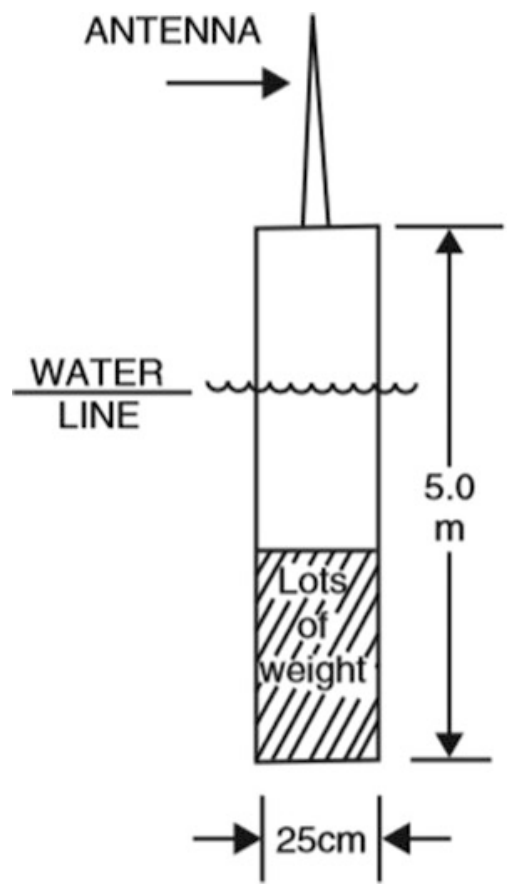

Fig. 2.36 The Earth with a hole passing through its diameter

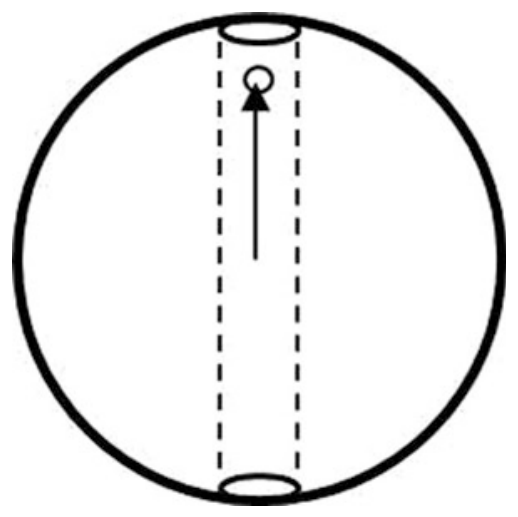

will be above or below the instantaneous (moving) air-sea interface? Report the amplitude of the motion of the buoy relative to the moving water surface, not with respect to the static (calm) water level.

6. Balls to Bunbury. Assume the Earth is the large diameter solid sphere of uniform mass density, $\rho_{\oplus}$, shown in Fig. 2.36. A small-diameter cylindrical hole has been bored through the sphere along a diameter. A ball of mass, $m$, with a diameter smaller than the hole, is dropped from the surface of the sphere down the hole.

At a distance, $r$, from the center of the Earth, the magnitude of the gravitational potential energy of the ball, $|U(r)|$, is determined by the mass of the Earth, $M(r)=4 \pi \rho r^{3} / 3$, which is included within a radius less than $r$. (Note the net force on the small mass due to the mass of the Earth that is at a radius greater than $r$ is completely cancelled. Why?) 
(a) Effective stiffness. Use Newton's Law of Universal Gravitation, as expressed in either Eq. (2.23) or Eq. (2.25), to determine an effective stiffness constant. Newton's Universal Gravitation Constant, $G=6.6726 \pm 0.0005 \times 10^{-11} \mathrm{~m}^{3} / \mathrm{s}^{2}-\mathrm{kg}$. For the planet Earth, $R_{\oplus}=6371 \mathrm{~km}$, the average mass density is $\rho_{\oplus}=5520 \mathrm{~kg} / \mathrm{m}^{3}$.

(b) Oscillation period. Find the period of oscillation. Express your answer in minutes.

(c) Orbital period. Using Eq. (2.23), show that the period you calculated in part (b) is identical to the period of the same mass (or any mass!) in a circular orbit around Earth at a height above the surface $h \ll R_{\oplus}$.

(d) Superposition. How is the linear motion of the mass through the Earth related to the orbital motion around the Earth?

7. An asymmetrically placed mass on a taut string. A mass, $m$, is attached on a string of tension, $\mathrm{T}$, and length, $L$. The mass is located a distance, $b$, from the left-side rigid support (hence $L-b$ from the right rigid support).

(a) Natural frequency. What is natural frequency if we assume that the string is massless, $m_{s}=0$ ?

(b) String mass correction. Using Rayleigh's method, calculate the natural frequency if $m_{s}=m / 10$, and report the result as a ratio with respect to the natural frequency you calculated in part (a).

8. Vibration isolator. A large air conditioning unit is loaded on to a vibration isolation platform. The platform that supports the chiller, which is attached to the isolators, sinks by $2.0 \mathrm{~cm}$ when the air conditioner is placed on it.

(a) Natural frequency. What is the natural frequency (in $\mathrm{Hz}$ ) of the chiller-isolator system? (You may assume that the acceleration due to gravity is $9.8 \mathrm{~m} / \mathrm{s}^{2}$.)

(b) Transmissibility. If the unbalance in the chiller's motor produces an oscillating force in the vertical direction of $F_{1}=1000 N_{\text {peak }}$ at $60 \mathrm{~Hz}$, what is the reduction in the force that the platform applies to the rigid foundation on which it mounted? Report the result as both a decimal ratio and as a $\mathrm{dB}$ reduction.

9. Cantilever spring. The beam in Fig. 2.37 was drawn by Galileo. When the $50 \mathrm{~kg}$ mass "E" was placed on the hook "C," the hook was displaced downward by $\Delta=4 \mathrm{~cm}$.

Fig. 2.37 Beam supported by a wall with mass "E"

attached at the end

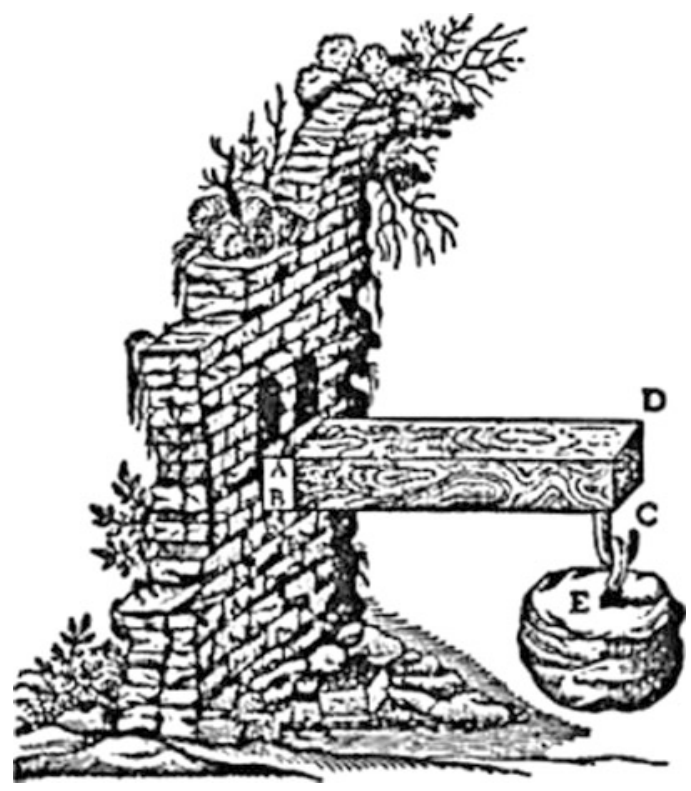


(a) Frequency. Neglecting the mass of the beam, what is the frequency of vibration?

(b) Beam mass correction. The deflection of the beam, $y(x)$, as measured from the rigid support at $x=0$ is given by the following curve (from Eq. (4.36):

$$
y(x)=\frac{3 \Delta}{2 L^{3}}\left(L x^{2}-\frac{x^{3}}{3}\right)
$$

If the mass of the beam is $10 \mathrm{~kg}$, use Rayleigh's method to recalculate the vibration frequency.

10. Atomic force microscopy. The beam in Fig. 2.38 is the sensing element of an atomic force microscope. ${ }^{31}$ The cantilever is fabricated from silicon nitride $\left(\mathrm{Si}_{3} \mathrm{~N}_{4}\right)$ and is $w=30 \mu \mathrm{m}$ wide, $L=100 \mu \mathrm{m}$ long, and $t=3 \mu \mathrm{m}$ thick. For this problem, the mass of the tip can be neglected.

The stiffness of this cantilever can be related to the Young's modulus of the $\mathrm{Si}_{3} \mathrm{~N}_{4}, E=310 \mathrm{GPa}$, by Eq. (2.143).

$$
\mathrm{K}=\frac{F}{\Delta}=\frac{E w}{4}\left(\frac{t}{L}\right)^{3}
$$

(a) Units. Show that the units on the right-hand side of Eq. (2.143) correspond to the units of stiffness.

(b) Stiffness. What is the value of the stiffness of the $\mathrm{Si}_{3} \mathrm{~N}_{4}$ micromachined cantilever?

(c) Thermal noise. What is the root-mean-square displacement, $x_{r m s}=\left\langle x^{2}\right\rangle^{1 / 2}$, of the tip if the cantilever is at a temperature of $20^{\circ} \mathrm{C}=293 \mathrm{~K}$ ?

(d) Minimum detectible force. What force on the tip would be required to produce a static deflection, $\Delta$, that is equal to the root-mean-square thermal fluctuation in the position of the tip, $x_{r m s}$, that you calculated in part (c).

Fig. 2.38 Silicon cantilever with spike

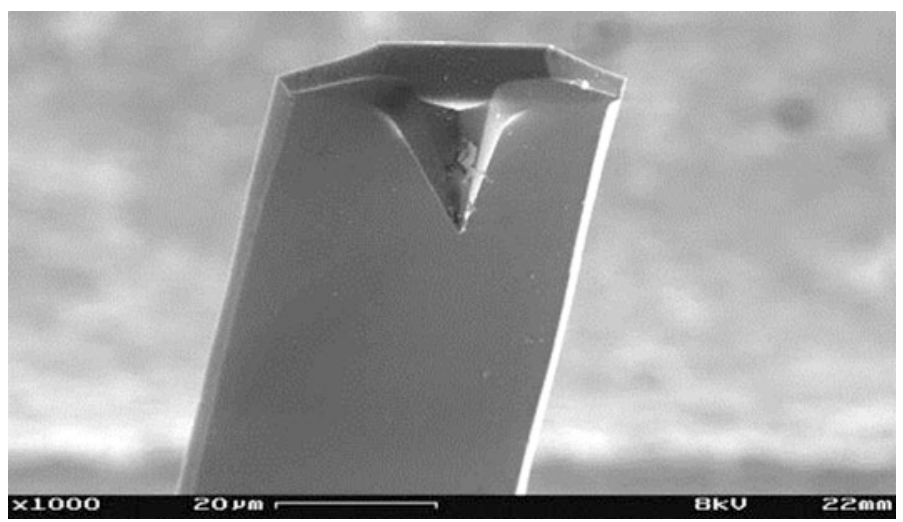

\footnotetext{
${ }^{31}$ Image courtesy of SecretDisk under their Creative Commons Attribution-Share Alike 3.0 license.
} 
Fig. 2.39 Two electrically charged spheres are constrained to move only in the vertical direction. The potential energy, $U(x)$, of the upper sphere is due to the combination of the force of gravity and the force of electrostatic repulsion.

(Figure courtesy of J. D. Maynard [23])
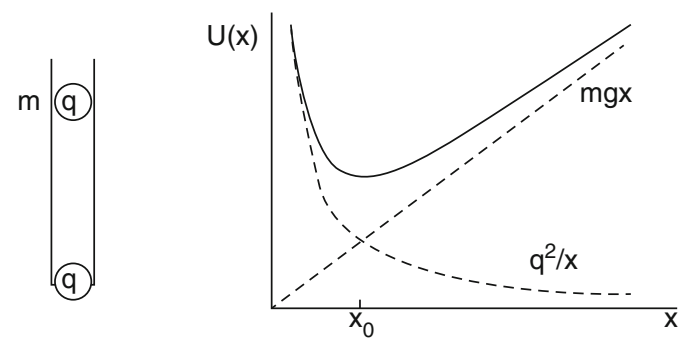

11. Electrostatic levitation. Two spheres, made of an electrically insulating material, are contained within a frictionless insulating tube as shown in Fig. 2.39. (You can imagine this as two tiny Styrofoam balls in a drinking straw.) The lower sphere is rigidly attached to the bottom of the tube, and the upper sphere is free to move up or down, without significant damping, only in the vertical direction.

The mass of each sphere is $0.10 \mathrm{~g}$. The electrostatic force, $F$, between the spheres is determined by the charges on the spheres, $Q_{\text {upper }}$ and $Q_{\text {lower }}$; the separation between their centers, $x$; and a constant, $\varepsilon_{o}=8.85 \times 10^{-12}$ Farads/meter, known as the permittivity of free space. In this case, the force between the spheres is repulsive, since the charge on both spheres is positive.

$$
F=\frac{1}{4 \pi \varepsilon_{o}} \frac{Q_{\text {upper }} Q_{\text {lower }}}{x^{2}}
$$

At equilibrium, the centers of the spheres are separated by $1.0 \mathrm{~cm}$. What is the frequency of oscillation of the upper sphere if it is displaced from its equilibrium position by a very small amount? (Let the acceleration due to gravity, $g=9.8 \mathrm{~m} / \mathrm{s}^{2}$.)

12. Lennard-Jones potential. This potential function, sometimes known as the " $6-12$ potential" of Eq. (2.145), is plotted in Fig. 2.40. It describes the interaction of two inert gas atoms as a function of their separation and is useful in calculations of scattering or determination of the temperature of condensation from a gas to a liquid. It was first proposed by John Lennard-Jones in 1924 [35].

$$
V(r)=4 \varepsilon\left[\left(\frac{\sigma}{r}\right)^{12}-\left(\frac{\sigma}{r}\right)^{6}\right]
$$

The term proportional to $r^{-12}$ represents an empirical fit to the hard-core repulsion produced by Pauli exclusion at short ranges when the atoms are close enough that the electron orbitals overlap. The $r^{-6}$ term represents the van der Waals attraction due to the mutual interactions of the fluctuating dipole moments.

(a) Equilibrium separation. Determine the equilibrium separation of two atoms, $r_{m}$, in terms of $\sigma$ and $\varepsilon$.

(b) Argon separation. For argon, $\sigma=3.42 \AA\left(1 \AA=10^{-10} \mathrm{~m}\right)$ and $\varepsilon=124 \mathrm{~K}$ $\left(1 \mathrm{~K}=1.38 \times 10^{-23} \mathrm{~J}\right)$. Determine the equilibrium separation of two argon atoms. 
Fig. 2.40 Lennard-Jones interatomic potential as a function of scaled atomic spacing, $r / \sigma$

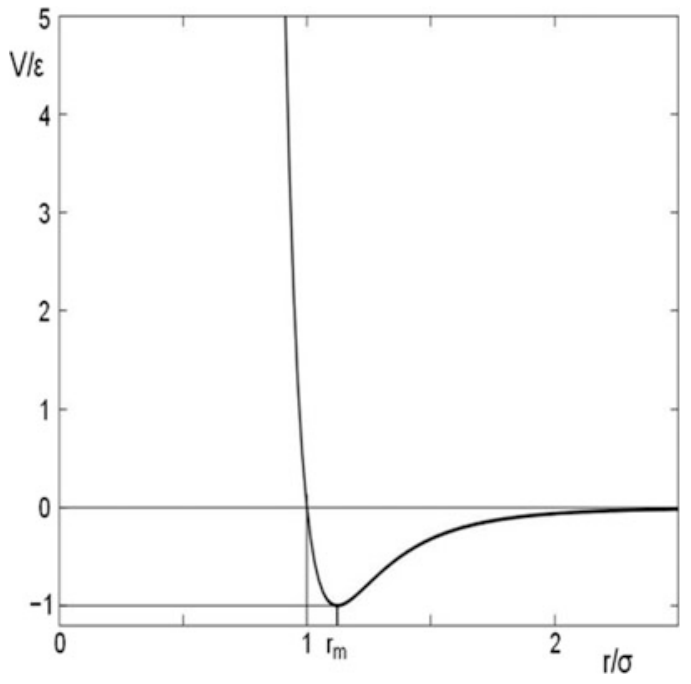

(c) Antisymmetric vibrational frequency. The atomic mass of an argon atom is $39.948 \mathrm{amu}$ $\left(1 \mathrm{amu}=1.66 \times 10^{-27} \mathrm{~kg}\right)$. Determine the stiffness constant, $\mathrm{K}=\mathrm{d}^{2} V / \mathrm{d} r^{2}$, and use that result to calculate the vibrational frequency of two isolated argon atoms about their equilibrium separation.

13. Ionic bonding. Hydrogen chloride $(\mathrm{HCl})$ is a diatomic molecule that forms an ionic bond. The force that bonds the $\mathrm{H}^{+}$ion to the $\mathrm{Cl}^{-}$is the electrostatic attraction between the oppositely charged ions. Of course, Pauli repulsion will separate the two ions if they are drawn too close together. The potential, $V(r)$, for this interaction is the sum of the electrostatic attraction and the Pauli repulsion.

$$
V(r)=-\frac{e^{2}}{4 \pi \varepsilon_{o} r}+\frac{B}{r^{9}}
$$

The permittivity of free space is $\varepsilon_{o}=8.85 \times 10^{-12}$ Farads/meter $[\mathrm{F} / \mathrm{m}]$, and the charge on a single electron is $e=1.602 \times 10^{-19} \mathrm{C}$.

(a) Repulsion constant. If the equilibrium separation of centers of the two ions is $r_{m}=1.30 \AA$ ( $\left.1 \AA=10^{-10} \mathrm{~m}\right)$, determine the value of the constant, $B$, in Eq. (2.146).

(b) Effective stiffness. What is the effective stiffness, $\mathrm{K}$, of the bond about the equilibrium separation, $r_{m}$ ?

(c) Vibrational frequency. The mass of the chlorine ion is about 35 times greater than the hydrogen ion. Assume that the position of chlorine atom is fixed, and determine the frequency of oscillation of the hydrogen ion if it is displaced by a small distance, $x_{1} \ll r_{m}$.

(d) Reduced mass. Since the mass of the chlorine ion is not infinite, the oscillation actually takes place about the center of mass, which remains at rest. This can be accounted for by using the reduced mass, $\mu$, given in Eq. (2.147), rather than using the mass of the hydrogen ion, so $f_{o}=(\mathrm{K} / \mu)^{1 / 2} / 2 \pi$.

$$
\mu=\frac{M_{C l^{-}} M_{H^{+}}}{M_{C l^{-}}+M_{H^{+}}}
$$


Fig. 2.41 Damped, driven harmonic oscillator

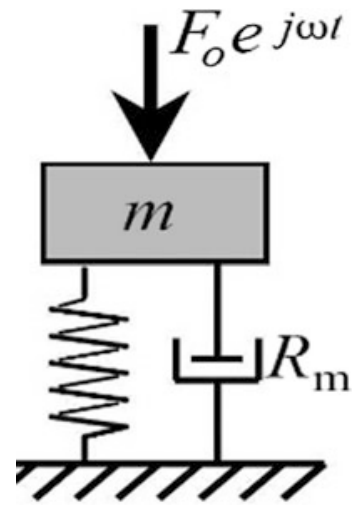

By what percentage is this frequency shifted from the value that assumed the chlorine atom had infinite mass?

14. Damped driven SHO. The system in Fig. 2.41 has a mass, $m=0.100 \mathrm{~kg}$, that is attached to a rigid foundation with a spring of stiffness, $K=1000 \mathrm{~N} / \mathrm{m}$, and a dashpot with mechanical resistance, $R_{m}$, that is to be determined in part (b) of this problem.

(a) Natural frequency. What is the natural frequency of the mass-spring system, in hertz, if the damper is not present $\left(R_{m}=0\right)$ ?

(b) Mechanical resistance. After an impulsive excitation, it is found that amplitude of free vibration of the mass decays to $e^{-1}$ of its value in $2.0 \mathrm{~s}$. Determine the value of $R_{m}$ and report your results in $\mathrm{kg} / \mathrm{s}$.

(c) Driven response. If the mass is driven with a time-harmonic force of amplitude $10 \mathrm{~N}$, at a frequency of $20 \mathrm{~Hz}, F(t)=10 e^{j 40 \pi t}$, what is:

(i) The magnitude of the time-harmonic displacement of the mass?

(ii) The phase of the mass's velocity with respect to that of the driving force?

(iii) The time-averaged power dissipated in $R_{m}$ ?

15. Damped harmonic oscillator. A $1.00 \mathrm{~kg}$ mass is suspended from a spring. A $0.40 \mathrm{~kg}$ mass is hung by a string below the $1.00 \mathrm{~kg}$ mass. The additional mass causes the spring to be extended by an additional $4.0 \mathrm{~cm}$. The string is cut at $t=0$ and the additional mass drops to the floor. The resulting harmonic oscillations of the $1.00 \mathrm{~kg}$ mass are observed to decay by $e^{-1}$ in $1 \mathrm{~s}$.

(a) Spring stiffness. What is the spring's stiffness?

(b) Mechanical resistance. What is the oscillator's mechanical resistance, $R_{m}$ ?

(c) Displacement. Equation (2.43) provides an expression for the motion of the mass, $x(t)$. Determine the values of the constants $C, \tau, \omega_{d}$, and $\phi$ that describe the motion for $t \geq 0$.

16. Five identical masses. Determine the five modal frequencies, and sketch the corresponding five mode shapes (like Fig. 2.31) for five masses, each with mass, $m$, that is equally spaced along a massless string of total length, $L=6 a$, that is fixed at both ends (i.e., $y(0)=y(6 a)=0$ ) and is stretched to a tension, T. You may assume that the vertical displacements of all of the masses are small compared to their separation (i.e., $y \ll a$ ). The normal mode frequencies can be reported in terms of T, $a$, and $m$.

17. Coupled oscillators. Nine objects of equal mass are joined by ten identical springs to two rigid boundaries as shown in Fig. 2.42. The masses are constrained to move along the line joining them. In Fig. 2.42, the equilibrium positions of the numbered masses are indicated by the equally spaced tick marks. In that diagram, the nine masses are shown at a particular phase of their collective 


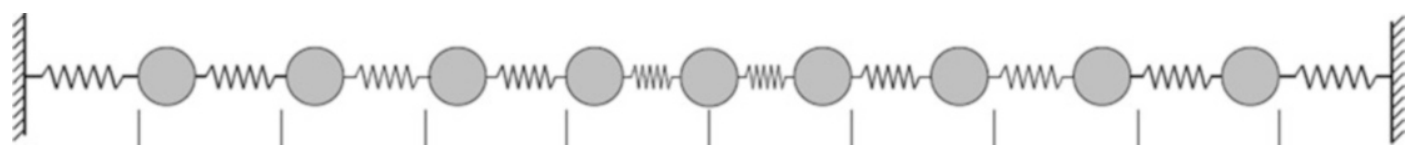

Fig. 2.42 Nine identical masses connected to rigid boundaries by nine identical springs

Fig. 2.43 Two pendula coupled by a spring

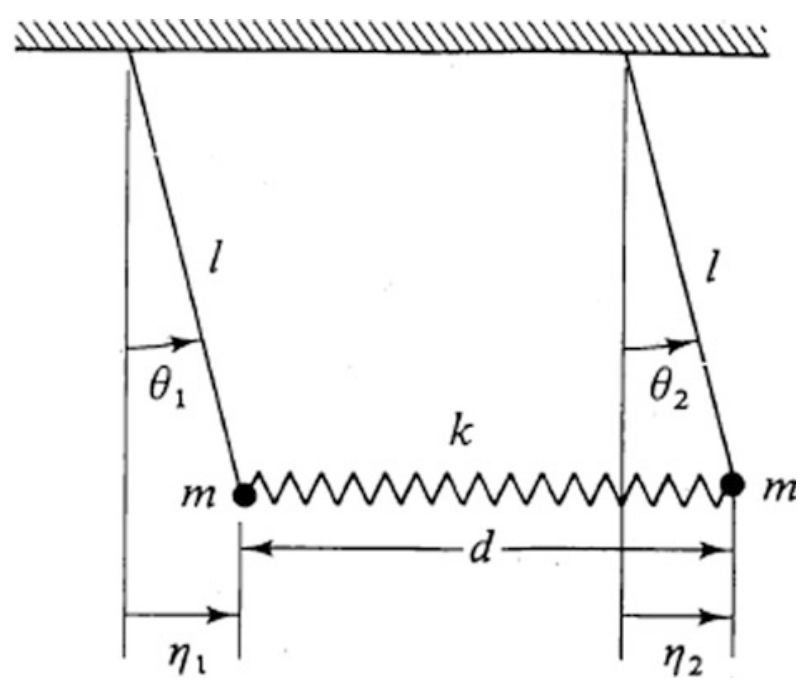

oscillation corresponding to a normal mode frequency of $20 \mathrm{~Hz}$. What is the frequency of the fundamental (lowest-frequency) mode of oscillation?

18. Two coupled pendula. Consider the two identical pendula with masses, $m$, and lengths, $\ell$, which are joined by a spring of stiffness, K, diagrammed in Fig. 2.43.

(a) Coupled equations. Assume that the angular displacements, $\theta_{1}$ and $\theta_{2}$, are both small enough that $\sin \theta \cong \theta$. Write the coupled differential equations that relate $\mathrm{d}^{2} \theta / \mathrm{d} t^{2}$ to the angular displacements.

(b) Modal frequencies. After making the harmonic substitution, calculate the determinate-ofcoefficients of the resulting coupled algebraic equations to determine the symmetric and antisymmetric normal mode frequencies in terms of $\ell, m, \mathrm{~K}$, and gravitational acceleration, $g$.

19. Electrodynamic loudspeaker. In this exercise, you will characterize a $5^{\prime \prime}$ electrodynamic loudspeaker (Morel MW 142, S/N 0496). You may neglect all acoustic loading and assume that the speaker measurements in Table 2.1 are made in a vacuum. Figure 2.44 contains the catalog description of the speaker (Right) and a diagram of its parts (Left) taken from Hunt's Electroacoustics [36].

You are to determine the values of the loudspeaker's parameters and their uncertainty using leastsquares curve fitting and error propagation using the measurements summarized in Table 2.1.

(a) Stiffness and moving mass. Determine the original moving mass, $m_{o}$, and dynamic stiffness, $\mathrm{K}$, of the loudspeaker and the uncertainties in those values based on the data in the first two columns of data at the left end of Table 2.1.

(b) Mechanical resistance. If the driver is driven at its resonance frequency and then the power is cut and the voice coil $\left(R_{d c}=5.1 \Omega\right)$ is connected to a high impedance load, the exponential free-decay time, $\tau$, can be related to the mechanical resistance, $R_{m}=2 m_{o} / \tau$, of the loudspeaker. The columns labeled "Time" and "V(peak)" in Table 2.1 are the results of 
Table 2.1 (Left to right) Measured frequency vs. added mass (shaded), free-decay amplitude vs. time, LVDT output vs. position (shaded), and LVDT output vs. DC electrical current through the voicecoil

\begin{tabular}{|c|c|c|c|c|c|c|c|}
\hline$\frac{\text { Mass }}{(\mathrm{gm})}$ & $\frac{\text { Freq. }}{(\mathrm{Hz})}$ & $\frac{\text { Time }}{(\mathrm{ms})}$ & $\frac{V(\text { peak })}{\left(\mathrm{mV}_{\mathrm{dc}}\right)}$ & $\frac{\text { Position }}{\text { (in.) }}$ & $\frac{\underline{V}_{\text {out }}}{\left(\mathrm{mV}_{\mathrm{dc}}\right)}$ & $\frac{\underline{I}_{d c}}{\left(m A_{d c}\right)}$ & $\frac{\underline{V}_{o u t}}{\left(\mathrm{mV}_{\mathrm{dc}}\right)}$ \\
\hline 0 & 55.5 & 29.25 & -823 & 0.700 & 947.7 & -253.3 & 388 \\
\hline 0.877 & 53.6 & 35.50 & 0 & 0.650 & 728.3 & -202.1 & 343 \\
\hline 2.877 & 49.9 & 39.55 & +318 & 0.600 & 512.4 & -153.6 & 301 \\
\hline 5.877 & 45.6 & 45.32 & 0 & 0.550 & 296.3 & -101.4 & 256 \\
\hline 10.877 & 40.2 & 49.40 & -144 & 0.500 & 81.4 & -54.6 & 216 \\
\hline \multirow[t]{5}{*}{20.877} & 33.4 & 54.97 & 0 & 0.450 & -133.5 & 0 & 169.1 \\
\hline & & 59.00 & +69 & 0.400 & -247.3 & +49.8 & 136.0 \\
\hline & & 64.47 & 0 & & & +102.6 & 91.3 \\
\hline & & 68.75 & -33 & & & +151.4 & 46.1 \\
\hline & & & & & & +202.5 & 1.6 \\
\hline
\end{tabular}

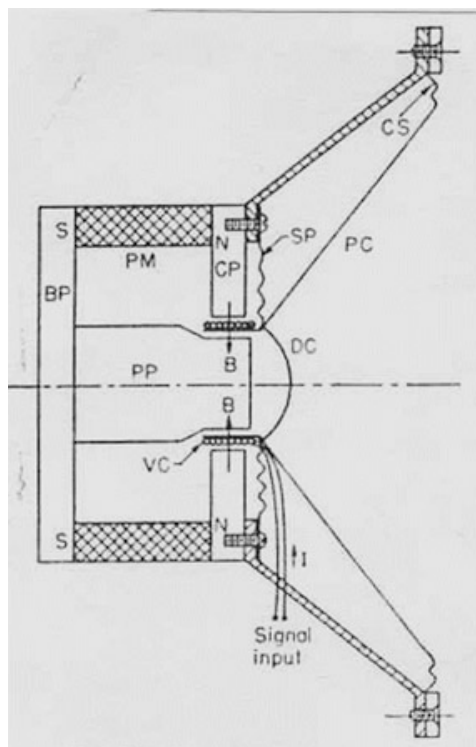

\section{MW-142 5" DPC Woofer}

A unique $5^{*}$ unit that incorporates a damped polymer composite cone, huge $3^{*}$ Hexatech voice coil, vented double magnet system, and smooth response up to $5 \mathrm{KHz}$. All this results in a very impressive bass/midrange driver.

$\diamond$ Power handling: 150 watts RMS/210 watts max $\bullet$ Voice

coil diameter: $3^{*}$ - Impedance: 8 ohms $\triangle D$ D resistance: 5.2

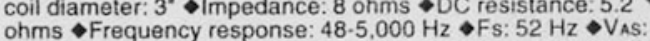

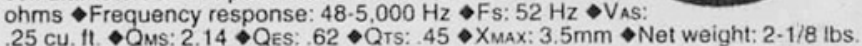
-Dimensions: A: $5 \cdot 5 / 8^{*}, \mathrm{~B}: 4-5 / 8^{*}, \mathrm{C}: 2-1 / 16^{\circ}, \mathrm{D}: 3-3 / 8^{\prime \prime}, \mathrm{E}: 1-1 / 4^{*}$.

\#297-020 $\$ 71.50_{(1-3)} \$ 68.90_{(4-11)} \$ 65.50_{(12-U P)}$

1. 5.1. Typiral structura! features of a loudsyraker of the moving-coil direct-radiato: type.

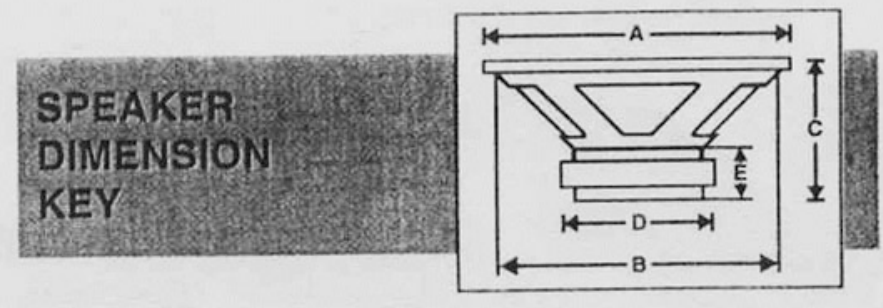

Fig. 2.44 Electrodynamic loudspeaker used to produce the results summarized in Table 2.1

such a free-decay measurement. $V$ (peak) is the value of the voltage at the peak (or trough) of each cycle. The peaks occur at integer multiples of the period $T, n=0,1,2, \ldots$ The troughs occur at half-odd integer values of $n=1 / 2,3 / 2,5 / 2, \ldots$

$$
V(\text { peak })=V_{1} e^{-n T / \tau}
$$

Take the natural $\log$ of that expression to convert to a linear relation, and find $R_{m}$ and its uncertainty by creating a plot like Fig. 1.15.

(c) LVDT calibration. To determine the transduction coefficient, $B \ell$, the displacement of the loudspeaker cone has been measured as a function of DC current, $I_{d c}$, through the voice coil: 



Fig. 2.45 (Above) Cross-sectional diagrams of an LVDT at the left and electrical schematic diagram of the astatic transformer showing the (driven) primary and (output) secondary coils. (Below) Position of the LVDT core displaced to the left, centered, and displaced to the right. (Courtesy of Lucas-Schaevitz)

$F=-\mathrm{K} x=-(B \ell) I_{d c}$. The displacement is measured by a linear variable differential transformer (LVDT) shown in Fig. 2.45.

The LVDT output (secondary) consists of two coils that are connected (electrically) in series but are wound in opposite directions. The movable core has a high magnetic permittivity. When the core is exactly at the center (null position), it couples an equal amount of the oscillating magnetic flux (produced by an AC current applied to the primary coil) to both secondary coils resulting in zero output voltage. When the core is in position $A$, more flux is coupled into the left-hand core which produces a waveform that has an amplitude that is proportional to the distance from the null position and is in-phase with the signal driving the primary. When the core is in position $B$, more flux is coupled into the right-hand core, producing a waveform amplitude that is also proportional to the distance from the null position but is out-of-phase with the signal driving the primary. The voltage output of one such LVDT as a function of position is tabulated in columns labeled "Position" and " $V_{\text {out }}$ " Plot $V_{\text {out }}$ vs. Position to determine the sensitivity of the LVDT, $\mathrm{d} V_{\text {out }} / \mathrm{d} x$, and its uncertainty.

(d) $B \ell$-product. After calibration of the LVDT, the $B \ell$-product can be determined from the fit to " $I_{d c}$ " and " $V_{\text {out }}$ " the LVDT calibration, and the measured stiffness constant, K, from part (a). Find the value of $B \ell$ and its uncertainty. ${ }^{32}$

(e) Constant current response. Assume the loudspeaker is driven by a constant sinusoidal current of $100 \mathrm{~mA}_{\mathrm{rms}}$ at all the frequencies of interest in this exercise. ${ }^{33}$ Plot the magnitude and phase of the voice coil's velocity, $\boldsymbol{v}$, as a function of frequency between $10 \mathrm{~Hz}$ and $1 \mathrm{kHz}$. Use a $\log _{10}$ axis for frequency.

(f) Power dissipation. Determine the power dissipated in the speaker's mechanical resistance, $R_{m}$, at the resonance frequency, $\omega_{o}$.

\footnotetext{
${ }^{32}$ This calculation assumes that the dynamic stiffness constant measured in the added mass experiment is the same as the static stiffness constant that is used in this part of the exercise to convert the displacement of the cone to the force produced by the current in the voice coil. Due to the viscoelastic behavior of the cone's surround, those two stiffnesses can differ due to relaxation of the elastomeric surround (see Fig. 1.16). For this exercise, that difference can be neglected.

${ }^{33}$ In normal operation, speakers are usually driven at constant voltage, but the variation in the speaker's electrical impedance with frequency makes that calculation more difficult.
} 
20. Jug hustlers. One of the most common tools in the oil exploration business is the geophone. It is used for seismic surveys, and the surveyors who plant and recover this ground vibration velocity sensors are known as "jug hustlers," since the geophones look like little jugs (see Fig. 2.24). In this problem, you will calculate the response of a GeoSpace ${ }^{\circledR}$ Corp. Model GS-30 CT geophone. The moving mass (mostly the coil and bobbin), $m_{o}=11.2 \mathrm{gm}( \pm 3.5 \%)$, and the natural frequency, $f_{o}=\omega_{o} / 2 \pi=10 \mathrm{~Hz}$.

(a) Damping. The open-circuit damping factor, $\zeta_{o}=0.316=(2 Q)^{-1}$. Find the mechanical resistance, $R_{m}$, of the moving coil and its relative uncertainty.

(b) Sensitivity. The electrodynamically generated, open-circuit voltage, $V_{\text {emf }}=(B \ell) v$, where $v$ is the relative velocity of the coil with respect to the case. If the transduction coefficient, $(B \ell)=70 \mathrm{~N} / \mathrm{A}$, calculate the root-mean-squared (rms) open-circuit voltage, $\mathrm{V}_{\text {rms }}$, generated by the geophone if the peak-to-peak ground motion is $10.0 \mu \mathrm{m}$ at $20,50,100$, and $200 \mathrm{~Hz}$.

(c) Thermal noise. Use the Equipartition Theorem to determine the value of $V_{r m s}$ due to the thermal motion of the coil if the temperature, $T=20^{\circ} \mathrm{C}$.

21. Rayleigh line-shape fit. The data in Table 2.2 provides the measured amplitude at 22 frequencies around resonance for the third (i.e., $n=3$ ) torsional mode of a cylindrical rod made from Eastman $\operatorname{Tritan}^{\circledR}$ Copolyester TX1001. Using the Rayleigh line-shape of Eq. (2.63), determine the best values for $f_{3}, A_{3}(1)$, and $Q_{3}$ by minimizing the squared difference between the measurements and the values produced by Eq. (2.63) by using your choice of "solver" software.

(a) Parameter estimates. The convergence of any solver's results will depend upon the quality of the initial guess for the parameters that the solver is allowed to vary. Estimate $f_{3}$ and $A_{3}(1)$ by choosing the largest amplitude value in Table 2.2 and its corresponding frequency. Estimate $Q_{3} \cong f_{3} /\left(f_{+}-f_{-}\right)$where $f_{+}$and $f_{-}$are the "down $3 \mathrm{~dB}$ " frequencies, as in Eq. (B.3).

Table 2.2 Resonance amplitude vs. frequency

\begin{tabular}{l|l}
\hline Frequency $[\mathrm{Hz}]$ & Amplitude $\left[\mathrm{mV}_{\mathrm{ac}}\right]$ \\
\hline 3245 & 682.5 \\
\hline 3260 & 714.9 \\
\hline 3275 & 749.7 \\
\hline 3290 & 787.1 \\
\hline 3305 & 827.4 \\
\hline 3320 & 870.1 \\
\hline 3335 & 915.3 \\
\hline 3350 & 957.1 \\
\hline 3380 & 997.2 \\
\hline 3395 & 1034.1 \\
\hline 3410 & 1059.0 \\
\hline 3425 & 1073.0 \\
\hline 3440 & 1074.7 \\
\hline 3455 & 1063.6 \\
\hline 3470 & 1036.4 \\
\hline 3485 & 998.0 \\
\hline 3515 & 953.3 \\
\hline 3530 & 905.1 \\
\hline 3545 & 855.9 \\
\hline 3560 & 806.1 \\
\hline & 758.7 \\
\hline
\end{tabular}


(b) Plot. On a single graph with properly labeled axes (including units), plot the data in Table 2.2 as "points" and the Rayleigh line shape as a smooth line using the parameter estimates of part (a).

(c) Optimize fit. Have your solver software optimized the values of $f_{3}, A_{3}(1)$, and $Q_{3}$ by minimizing the squared difference between the measurements in Table 2.2 and the values produced by Eq. (2.63). Report the optimized values, and update your plot in part (b) using the best-fit parameter values.

(d) Error estimate. How could you try to estimate the uncertainty in the fit parameters? Use your scheme to report your estimate in the relative uncertainty $( \pm 1 \sigma)$ for your optimized values of $f_{3}, A_{3}(1)$, and $Q_{3}$.

\section{References}

1. R.C. Cross, M.S. Wheatland, Modeling a falling slinky. Am. J. Phys. 80(12), 1051-1060 (2012)

2. H. Goldstein, Classical Mechanics (Addison-Wesley, Reading, 1950), pp. 69-71

3. J.W. Strutt (Lord Rayleigh), The Theory of Sound, vol. I, 2nd edn. (Macmillan, London, 1894), pp. 112; reprinted (Dover, 1945): ISBN: 486-60292-3

4. J.W. Strutt (Lord Rayleigh), The Theory of Sound, vol. I, 2nd edn. (Macmillan, London, 1894), pp. 109-110; reprinted (Dover, 1945): ISBN: 486-60292-3

5. G.W. Swift, Thermoacoustics: A Unifying Perspective for Some Engines and Refrigerators, 2nd edn. (ASA Press/ Springer, Cham, 2017); ISBN: 978-3-319-66932-8

6. M. Greenspan, Simple derivation of the Boltzmann-Ehrenfest adiabatic principle. J. Acoust. Soc. Am. 27(1), 34-35 (1955)

7. T.G. Giallorenzi, J.A. Bucaro, A. Dandridge, G.H. Sigel Jr., J.H. Cole, S.C. Rashleigh, P.G. Priest, Optical fiber sensor technology. IEEE J. Quantum Electron. QE-18(4), 626-665 (1982)

8. A. Einstein, Über die von der molekularkinetischen Theorie der Wärme geforderte Bewegung von in ruhenden Flüssigkeiten suspendierten Teilchen (On the motion of small particles suspended in a stationary liquid, as required by the molecular kinetic theory of heat). Ann. Phys. 17(8), 549-560 (1905)

9. W. Gerlach, E. Lehrer, Uber die Messung der rotatorishen Brownschen Bewegung mit Hilfe einer Drehwage. Naturwiss 15(1), 15 (1927)

10. G.E. Uhlenbeck, S. Goudsmit, A problem in Brownian motion. Phys. Rev. 34, 145-151 (1929)

11. P. Horowitz, W. Hill, The Art of Electronics, 2nd edn. (Cambridge University Press, Cambridge, 1989). §7.11; ISBN: 0-521-37095-7

12. H. Nyquist, Thermal agitation of electric charge in conductors. Phys. Rev. 32, 110-113 (1928)

13. C.W. McCombie, Fluctuation theory in physical measurements. Rep. Prog. Phys. 16, 266-319 (1953)

14. T.J. Hofler, S.L. Garrett, Thermal noise in fiber optic sensors. J. Acoust. Soc. Am. 84(2), 471-475 (1988)

15. D.L. Gardner, T. Hofler, S.R. Baker, R.K. Yarber, S.L. Garrett, A fiber optic interferometric seismometer. J. Lightwave Technol. LT-5(7), 953-960 (1987); S.L. Garrett, D.L. Gardner, Multiple axis fiber optic interferometric seismic sensor. U.S. Patent No. 4,893,930 (January 16, 1990)

16. F. Reif, Fundamentals of Statistical Mechanics and Thermal Physics (McGraw-Hill, New York, 1965), §7.5

17. W.P. Allis, M.A. Herlin, Thermodynamics and Statistical Mechanics (McGraw-Hill, New York, 1952)

18. T.B. Gabrielson, Mechanical-thermal noise in micromachined acoustic and vibration sensors. IEEE Trans. Electron Devices 40(3), 903-909 (1993)

19. A.B. Pippard, The Physics of Vibration (Cambridge University Press, Cambridge, 1989); ISBN: 0-521-37200-3

20. I.S. Gradshteyn, I.M. Ryzhik, Tables of Integrals, Series, and Products (Academic, London, 1980). isbn:0-12294760-6. See equation 3.264(2)

21. R.S. Wakeland, Use of electrodynamic drivers in thermoacoustic refrigerators. J. Acoust. Soc. Am. 107(2), 827-832 (2000)

22. R.E. Best, Phase-Locked Loop: Design, Simulation, and Applications, 6th edn. (McGraw-Hill, New York, 2007): ISBN: 9780079375-8

23. J.D. Maynard, Topics in physics, Part IV-Acoustics, Penn State Lecture Notes, 2012

24. P. Campbell, Permanent Magnet Materials and Their Application (Cambridge University Press, Cambridge, 1994); ISBN: 0521566886

25. S.L. Garrett, J.F. Heake, Hey kid! Wanna build a loudspeaker? The first one's free. Audio Engineering Society Convention Paper 5882 (2003 October 10-13, New York, NY); S.L. Garrett, Two-way loudspeaker enclosure 
assembly and testing as a freshman seminar. Paper \#526,17th International Congress on Sound and Vibration, 18-22 July 2010, Cairo, Egypt

26. T.W. Leishman, B.E. Anderson, Evaluation of moving-coil loudspeaker and passive radiator parameters using normal-incidence sound transmission measurements: Theoretical developments. J. Acoust. Soc. Am. 134(1), 223-236 (2013)

27. L.L. Beranek, Acoustics (Acoustical Society of America, Woodbury, 1996). pp. 185-188, 211-230: ISBN: 0-88318494-X

28. A.N. Thiele, Loudspeakers in vented boxes: Parts I and II. Proc. Inst. Radio Eng. (Australia) 22, 487-502 (1961); reprinted in J. Audio Eng. Soc. 19, 382-392, 471-483 (1971)

29. B. Baumzweiger (a.k.a., Bauer), Microphone apparatus, U.S. Patent No. 2,184,247 (December 19, 1939)

30. W.L. Dooley, Ribbon microphones. J. Acoust. Soc. Am. 136, 2130 (2014)

31. M.S. Pettersen, 75th anniversary of the Shure Unidyne ${ }^{\circledR}$ microphone. J. Acoust. Soc. Am. 136, 2129 (2014)

32. J.C. Snowdon, Vibration and Shock in Damped Mechanical Systems (Wiley, London, 1968)

33. P.M. Morse, Vibration and Sound, 2nd edn. (McGraw-Hill, New York, 1948). Reprinted (Acoustical Society of America, 1976). isbn:0-88318-287-4. The normal mode displacement are given in Eqs. (7.3) though (7.5), pp. 55-58

34. A.L. Fetter, J.D. Walecka, Theoretical Mechanics of Particles and Continua (Dover, Mineola, 2003); ISBN: 0-486-43261-0

35. J.E. Lennard-Jones, On the determination of molecular fields. Proc. R. Soc. Lond. A 106, $463-477$ (1924)

36. F.V. Hunt, Electroacoustics: The Analysis of Transduction and Its Historical Background (Wiley, New York, 1954); reprinted (Acoust. Soc. Am., 1982). ISBN: 0-88318-401-X

Open Access This chapter is licensed under the terms of the Creative Commons Attribution 4.0 International License (http://creativecommons.org/licenses/by/4.0/), which permits use, sharing, adaptation, distribution and reproduction in any medium or format, as long as you give appropriate credit to the original author(s) and the source, provide a link to the Creative Commons license and indicate if changes were made.

The images or other third party material in this chapter are included in the chapter's Creative Commons license, unless indicated otherwise in a credit line to the material. If material is not included in the chapter's Creative Commons license and your intended use is not permitted by statutory regulation or exceeds the permitted use, you will need to obtain permission directly from the copyright holder. 